\title{
Analysis of Coal by Static Time-of-Flight Secondary Ion Mass Spectrometry (TOF-SIMS)
}

\author{
Lei Pei \\ Brigham Young University - Provo \\ Guilin Jiang \\ Brigham Young University - Provo \\ Larry Lin Baxter \\ Brigham Young University - Provo, larry_baxter@byu.edu \\ Matthew R. Linford \\ Brigham Young University - Provo
}

Follow this and additional works at: https://scholarsarchive.byu.edu/facpub

Part of the Chemical Engineering Commons

\section{Original Publication Citation}

Pei, L., Jiang, G., Baxter, L. L., \& Linford, M. R. (2010). Analysis of coal by static time-of-flight secondary ion mass spectrometry (TOF-SIMS). Surface Science Spectra, 17(1): 1-67

\section{BYU ScholarsArchive Citation}

Pei, Lei; Jiang, Guilin; Baxter, Larry Lin; and Linford, Matthew R., "Analysis of Coal by Static Time-of-Flight Secondary lon Mass Spectrometry (TOF-SIMS)" (2011). Faculty Publications. 1733.

https://scholarsarchive.byu.edu/facpub/1733

This Peer-Reviewed Article is brought to you for free and open access by BYU ScholarsArchive. It has been accepted for inclusion in Faculty Publications by an authorized administrator of BYU ScholarsArchive. For more information, please contact ellen_amatangelo@byu.edu. 


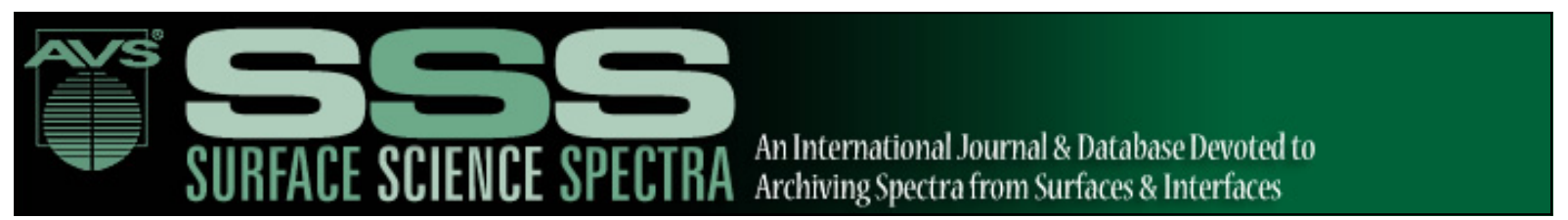

Analysis of Coal by Static Time-of-Flight Secondary lon Mass Spectrometry (TOF-SIMS)

Lei Pei, Guilin Jiang, Larry L. Baxter, and Matthew R. Linford

Citation: Surface Science Spectra 17, 1 (2010); doi: 10.1116/11.20080402

View online: http://dx.doi.org/10.1116/11.20080402

View Table of Contents: http://scitation.aip.org/content/avs/journal/sss/17/1?ver=pdfcov

Published by the AVS: Science \& Technology of Materials, Interfaces, and Processing

\section{Articles you may be interested in}

Structural analysis of negative ions by postsource decay in time-of-flight secondary ion mass spectrometry J. Vac. Sci. Technol. B 34, 03 H133 (2016); 10.1116/1.4944955

Analysis of Poly(amino acids) by Static Time-of-Flight Secondary lon Mass Spectrometry (TOF-SIMS)

Surf. Sci. Spectra 8, 163 (2001); 10.1116/11.20020301

Time-of-flight static secondary ion mass spectrometry of additives on polymer surfaces

J. Vac. Sci. Technol. A 9, 1307 (1991); 10.1116/1.577617

MASTIF: Mass analysis of secondaries by time-of-flight technique. A new approach to secondary ion mass spectrometry

Rev. Sci. Instrum. 60, 3188 (1989); 10.1063/1.1140550

Time-of-flight secondary ion mass spectrometry of polymer materials

J. Vac. Sci. Technol. A 7, 1790 (1989); 10.1116/1.576047

\section{HIDEN}

\section{Instruments for Advanced Science}

\section{Contact Hiden Analytical for further details: w www.HidenAnalytical.com E info@hiden.co.uk}

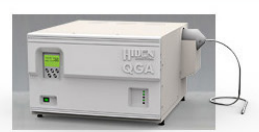

Gas Analysis

dynamic measurement of reaction gas streams , catalysis and thermal analysis dissolved species probes fermentation, environmental and ecological studies

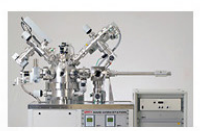

Surface Science UHVTPD SIMS

end point detection in ion beam etch elemental imaging-surface mapping

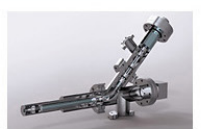

Plasma Diagnostics - plasma source characterization etch and deposition process reaction kinetic studies analysis of neutral and radical species

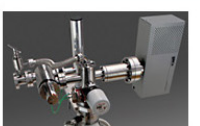

Vacuum Analysis partial pressure measurement and control of process gases reactive sputter process control vacuum diagnostics vacuum coating process monitoring 


\section{Analysis of Coal by Static Time-of-Flight Secondary Ion Mass Spectrometry (TOF-SIMS)}

Lei Pei and Guilin Jiang

Brigham Young University, Chemistry and Biochemistry, C100 BNSN, Provo, UT 84602

Larry L. Baxter

Brigham Young University, Chemical Engineering, 350 CB, Provo, UT 84602

Matthew R. Linford ${ }^{\text {a) }}$

Brigham Young University, Chemistry and Biochemistry, Cl00 BNSN, Provo, UT 84602

(Received 5 April 2008; accepted 21 January 2010; published 7 November 2011)

Coal remains a primary fuel for power generation. Herein we present time-of-flight secondary ion mass spectra (ToF-SIMS data) taken with a Ga primary ion beam from ca. 30 coal specimens. These commercially different coal specimens were obtained from coal mining companies and/or power plants. They represent all major coal types used in power generation (bituminous coals, subbituminous coals, and lignites), and include low-rank materials (lignites and subbituminous coals), which are represented as a minor portion of the data. Often, inorganic ions $\left(\mathrm{Na}^{+}, \mathrm{Al}^{+}, \mathrm{Si}^{+}\right.$, and $\mathrm{K}^{+}$) are pronounced in the spectra, overshadowing peaks from organic moieties. This reflects the high sensitivity of SIMS under our analysis conditions for these inorganic species. These results, including a previous, published chemometrics analysis of this data (L. Pei, G. Jiang, B. J. Tyler, L. L. Baxter, and M. R. Linford, Energy \& Fuels 2008, 22, 1059), suggest that ToF-SIMS can be a useful method for coal analysis. (C) 2010 American Vacuum Society.

[DOI: 10.1116/11.20080402]

\begin{tabular}{l} 
Accession \#s 01112, 01113, \\
01114, 01115, 01116, 01117, \\
01118, 01119, 01120, 01121, \\
01122, 01123, 01124, 01125, \\
01126, 01127, 01128, 01129, \\
01130, 01131, 01132, 01133, \\
01134, 01135, 01136, 01137, \\
01138, 01139, 01140, 01141, \\
01142, 01143, 01144, 01145, \\
01146, 01147 \\
Technique: ToF-SIMS \\
Host Material: \#01112: coal SS01; \\
\#01113: coal SS02; \#01114: coal \\
SS03; \#01115: coal SS04; \#01116: \\
coal SS05; \#01117: coal SS06; \\
\#01118: coal SS07; \#01119 coal \\
SS08; \#01120: coal SS09; \#01121: \\
coal SS10; \#01122: coal SS11; \\
\#01123: coal SS12; \#01124: coal \\
SS13; \#01125: coal SS14; \#01126: \\
coal SS15; \#01127: coal SS16; \\
\#01128: coal SS17; \#01129: coal \\
SS18; \#01130: coal SS19; \#01131: \\
coal BT; \#01132: coal CJR012; \\
\#01133: coal GAT034; \#01134: \\
coal GAT035; \#01135: coal \\
GBJ010; \#01136: coal GLJ005; \\
\#01137: coal GPB062; \#01138: \\
coal HGB011; \#01139: coal \\
HNA015; \#01140: coal ILL6; \\
\#01141: coal JIG014; \#01142: coal \\
JIG017; \#01143: coal PCT010; \\
\#01144: coal PCT012; \#01145: \\
coal RBL014; \#01146: coal \\
RLL012; \#01147: coal RLL014; \\
Instrument: ION-TOF TOF-SIMS IV \\
Major Elements in Spectrum: C, H \\
Minor Elements in Spectrum: \\
Printed Spectra: 57 \\
Spectra in Electronic Record: 155 \\
Spectral Category: technical \\
\hline
\end{tabular}

\section{INTRODUCTION}

ToF-SIMS (time-of-flight secondary ion mass spectrometry) (Ref. 1) is a highly sophisticated and powerful analytical method that is currently undergoing constant improvements in both its equipment and data analysis. Indeed, SIMS has been applied to numerous interfaces and materials, including coal (Refs. 2-6). ToF-SIMS is conceptually simple; a surface is first irradiated with a primary ion beam. Both the positive and negative secondary ions that are generated as a result of these collisions can then be mass analyzed. The resulting secondary ions are often rich in chemical information about the original surface or material.

Herein we report a ToF-SIMS study of ca. 30 different coal samples. This contribution follows a paper we recently published (L. Pei, G. Jiang, B. J. Tyler, L. L. Baxter, and M. R. Linford, Energy \& Fuels 2008, 22, 1059), which presents a chemometrics (statistical)

a) Author to whom correspondence should be addressed. analysis of the raw data presented in this work. For our analysis, both the positive and negative ion spectra were, in general, collected on each sample at three different $500 \mu \mathrm{m} \times 500 \mu \mathrm{m}$ spots. For the most part, these replicate analyses were very similar, although in some cases the spectra suggested chemical differences between the analysis regions. This result seems reasonable given the inherent complexity of coal even after our careful grinding/sample preparation. The positive ion spectra of the coal samples often show strong inorganic peaks from $\mathrm{Na}^{+}, \mathrm{K}^{+}, \mathrm{Si}^{+}, \mathrm{Al}^{+}, \mathrm{Ca}^{+}, \mathrm{Fe}^{+}$, etc., along with other organic/hydrocarbon signals. Some of the spectra also contain a peak at $\mathrm{m} / \mathrm{z}=180$, which may have the formula $\mathrm{C}_{14} \mathrm{H}_{12}$, and/or $\mathrm{C}_{12} \mathrm{H}_{8} \mathrm{~N}_{2}$ (Ref. 7). The signal at $\mathrm{m} / \mathrm{z}=105$ is also unknown (it is not labeled in the spectra) and may be due to $\mathrm{C}_{7} \mathrm{H}_{5} \mathrm{O}$ and/or $\mathrm{C}_{8} \mathrm{H}_{9}$. The main peaks in the negative ion spectra are at $\mathrm{m} / \mathrm{z}=0-20$, where $\mathrm{H}^{-}$, $\mathrm{CH}^{-}, \mathrm{O}^{-}$, and $\mathrm{OH}^{-}$are often prominent. Of course, peak intensities do vary somewhat from analysis region to analysis region. Because of the similarity between the negative ion spectra, only one such spectrum (1112-3) is published in this contribution as a representative 
example. (Nevertheless, the remaining negative ion spectra (and similarly positive ion spectra) are archived with this SSS contribution.)

More traditional analyses were also used to characterize coal for the organic $(\mathrm{C}, \mathrm{H}, \mathrm{O}, \mathrm{N}, \mathrm{S})$ and inorganic $(\mathrm{Si}, \mathrm{Al}, \mathrm{Fe}, \mathrm{Ti}, \mathrm{Na}$, $\mathrm{K}, \mathrm{Ca}, \mathrm{Mg}, \mathrm{S}, \mathrm{P}$ ) ash-forming compounds, the moisture content, the heat of combustion, the softening/melting behavior under oxidizing and reducing conditions, and also the degree of thermal decomposition under standard conditions. Of these measurements, only the moisture is directly tied to a chemical entity and not an element, where this lack of chemical information makes it challenging to predict the thermal decomposition and/or chemical reactions that these fuels experience upon combustion. In other words, such highly complex, and often multiphase reactions are a function of the speciation and/or chemical natures of the fuels, which characteristics cannot typically be predicted solely on elemental analyses. In our work we have found that our ToF-SIMS results often correlate with the results from the more traditional analyses of the coal specimens. As an example, the samples that produce larger amounts of ash have ToF-SIMS spectra with more pronounced inorganic signals. Thus, the purpose of our investigation is to understand the degree to which SIMS can provide additional information regarding coal composition, which will lead to a deeper understanding of its combustion.

SPECIMEN DESCRIPTION (ACCESSION \#S 01112, 01113, 01114, 01115, 01116, 01117, 01118, 01119, 01120, 01121, 01122, 01123, 01124, 01125, 01126, 01127, 01128, 01129, 01130, 01131, 01132, 01133, 01134, 01135, 01136, 01137, 01138, 01139, 01140, 01141, 01142, 01143, 01144, 01145, 01146, 01147)

Host Material: Coal

CAS Registry \#: N/A

Host Material Characteristics: inhomogeneous, solid, amorphous, composite, powder

Chemical Name: Coal

Source: Alliant Power Systems, Southern Company and Tennessee Valley

Host Composition: C, H, O, Na, K, Si, Al, Ca, Fe

Form: powder

\section{Structure: N/A}

History and Significance: Fuel rank is undoubtedly the most common descriptor of coal. Rank increases in the order of lignite, subbituminous coal, bituminous coal, and anthracite, with the middle two ranks representing by far the most significant division in terms of coal. There are subranks within each division. The following shows the rank of each coal sample. SS01: a medium volatile bituminous coal. SS02: a semianthracite coal. SS03: a high volatile B bituminous coal. SS04: a low volatile bituminous coal. SS05: a low volatile bituminous coal. SS06: a medium volatile bituminous coal. SS07: a medium volatile bituminous coal. SS08: a low volatile bituminous coal. SS09: a high volatile A bituminous coal. SS10: a medium volatile bituminous coal. SS11: a subbituminous B coal. SS12: a subbituminous C coal. SS13: a subbituminous C coal. SS14: a subbituminous C coal. SS15: a lignite A coal. SS16: a high volatile B bituminous coal. SS17: a high volatile B bituminous coal. SS17: a high volatile A bituminous coal. SS19: a high volatile B bituminous coal. Blackthunder: a subbituminous coal. CJR012: a high volatile A bituminous coal. GAT034: a medium volatile bituminous coal. GAT035: a medium volatile bituminous coal. GBJ010:a high volatile A bituminous coal. GLJ005: a high volatile A bituminous coal. GPB062: a high volatile B bituminous coal. HGB011: a high volatile B bituminous coal. HNA015: a high volatile A bituminous coal. ILL6: a bituminous coal. JIG014: a high volatile A bituminous coal. JIG017: a medium volatile bituminous coal. PCT010: a high volatile A bituminous coal. PCT012: a medium volatile bituminous coal. RBL014: a high volatile A bituminous coal. RLL012: a medium volatile bituminous coal. RLL014: a high volatile A bituminous coal.

As Received Condition: powder in a sealed bottle

Analyzed Region: same as host material

Ex Situ Preparation/Mounting: ground into fine powder with a mortar and pestle, then mounted onto a silicon substrate with double-sided tape

\section{In Situ Preparation: N/A}

Charge Control: A charge control flood gun was used. Anode voltage $300 \mathrm{~V}$; Wehnelt voltage $73 \mathrm{~V}$; current $2.2 \mathrm{~A}$; no target bias or metal screen. The same charge control conditions are used for all non-conductive samples.

Temp. During Analysis: $298 \mathrm{~K}$

Pressure During Analysis: $<2.0 \times 10^{6} \mathrm{~Pa}$

\section{INSTRUMENT DESCRIPTION}

Manufacturer and Model: ION-TOF TOF-SIMS IV

Analyzer Type: time-of-flight, reflectron

Experiment Type: static

Charge Control/conditions and Procedures: A charge control flood gun was used. Anode voltage $300 \mathrm{~V}$; Wehnelt voltage $73 \mathrm{~V}$; current $2.2 \mathrm{~A}$; no target bias or metal screen. The same charge control conditions are used for all non-conductive samples.

Sample Voltage Offset Range: sample was grounded

Energy Acceptance Window: n/a

Detector Description: electron multiplier

Detected Sample Dimensions: $500 \mu \mathrm{m} \times 500 \mu \mathrm{m}$

Live Time: $100 \%$

Analyzer Mass Resolution: $\sim 5000 \mathrm{~m} / \Delta \mathrm{m}$

Mass Used to Determine Resolution: $28 \mathrm{Da}$

Sample Bias: $0 \mathrm{~V}$

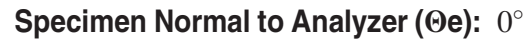

ION GUNS:

Number of Ion Guns Used: 1

Ion Gun No.: 1

Purpose: primary beam

Manufacturer and Model: ION-TOF 2 lens Ga gun

Beam Mass Filter: none

Beam Species: $\mathrm{Ga}^{+}$

Beam Gating Used: yes

Beam Comment: blanked and then bunched 
Beam Voltage: 25,000 V

Net Beam Voltage: $25,000 \mathrm{~V}$

Ion Pulse Length: $25 \times 10^{-9} \mathrm{sec}$

Ion Pulse Rate: $10,000 \mathrm{~Hz}$

Beam Current: $1.5 \mathrm{nA}$

Current Measurement Method: Faraday Cup

Beam Diameter: $0.1 \mu \mathrm{m}$

Beam Raster Size: $500 \mu \mathrm{m} \times 500 \mu \mathrm{m}$

Beam Incident Angle: $45^{\circ}$

Effective Beam Incident Angle: $45^{\circ}$

Source to Analyzer Angle: $45^{\circ}$

Specimen Azimuthal Angle: $45^{\circ}$

\section{CALIBRATION}

Mass Range Calibration: Calibration was based on the peaks $\mathrm{H}^{+}, \mathrm{C}^{+}, \mathrm{CH}^{+}, \mathrm{CH}_{2}{ }^{+}, \mathrm{CH}_{3}{ }^{+}, \mathrm{Na}^{+}, \mathrm{Si}^{+}, \mathrm{K}^{+}, \mathrm{Ga}^{+}$for positive spectra; $\mathrm{H}^{-}, \mathrm{C}^{-}, \mathrm{CH}^{-}, \mathrm{O}^{-}, \mathrm{OH}^{-}, \mathrm{C}_{2}{ }^{-},{ }^{35} \mathrm{Cl}^{-}, \mathrm{C}_{3}{ }^{-},{ }^{37} \mathrm{Cl}^{-}$for negative spectra

Detector Saturation: n/a

\section{ACKNOWLEDGMENTS}

We acknowledge Alliant Power Systems, Southern Company, and Tennessee Valley Authority for providing samples of coal. Financial support comes from different industrial sponsors of one of the author's (LLB) research.

\section{REFERENCES}

1. ToF-SIMS. Surface Analysis by Mass Spectrometry (IM Publications, Huddersfield, UK, 2001).

2. B. Gong, P. J. Pigram, and R. N. Lamb, Applied Occupational and Environmental Hygiene, 14, 481-489 (1999).

3. L. Pei, G. Jiang, B. J. Tyler, L. L. Baxter, and M. R. Linford, Energy and Fuel, 22, 1059 (2008).

4. T. Sakamoto, K. Shibata, K. Takanashi, M. Owari, and Y. Nihei, e-Journal of Surface Science and Nanotechnology, 2, 45-51 (2004).

5. G. Domazetis, M. Raoarun, B. D. James, and J. Liesegang, Energy \& Fuels, 19, 1047-1055 (2005).

6. G. Domazetis, M. Raoarun, B. D. James, J. Liesegang, P. J. Pigram, N. Brack, and R. Glaisher, Energy \& Fuels, 20, 1556-1564 (2006).

7. M. Novotny, D. Wiesler, and F. Merli, Chromatographia, 15, 374-377. 


\begin{tabular}{|c|c|c|c|c|}
\hline \multicolumn{5}{|c|}{ SPECTRAL FEATURES TABLE } \\
\hline Spectrum ID \# & Species & Source & Mass & Label \\
\hline $1112-1$ & $\mathrm{Al}$ & SS01 & 26.98 & $\mathrm{Al}$ \\
\hline $1112-1$ & $\mathrm{Si}$ & SS01 & 27.98 & $\mathrm{Si}$ \\
\hline $1112-1$ & $\mathrm{~K}$ & SS01 & 38.96 & $\mathrm{~K}$ \\
\hline $1112-1$ & $\mathrm{C}_{3} \mathrm{H}_{5}$ & SS01 & 41.04 & $\mathrm{C}_{3} \mathrm{H}_{5}$ \\
\hline $1112-1$ & $\mathrm{Fe}$ & SS01 & 55.94 & $\mathrm{Fe}$ \\
\hline $1112-1$ & $\mathrm{C}_{14} \mathrm{H}_{12}$ or $\mathrm{C}_{12} \mathrm{H}_{8} \mathrm{~N}_{2}$ & SS01 & 180.08 & $\mathrm{C}_{14} \mathrm{H}_{12}$ or $\mathrm{C}_{12} \mathrm{H}_{8} \mathrm{~N}_{2}$ \\
\hline $1112-2$ & Al & SS01 & 26.98 & $\mathrm{Al}$ \\
\hline $1112-2$ & $\mathrm{Si}$ & SS01 & 27.98 & $\mathrm{Si}$ \\
\hline $1112-2$ & $\mathrm{~K}$ & SS01 & 38.96 & K \\
\hline $1112-2$ & $\mathrm{C}_{3} \mathrm{H}_{5}$ & SS01 & 41.04 & $\mathrm{C}_{3} \mathrm{H}_{5}$ \\
\hline $1112-2$ & $\mathrm{Fe}$ & SS01 & 55.94 & $\mathrm{Fe}$ \\
\hline $1112-2$ & $\mathrm{C}_{4} \mathrm{H}_{9}$ & SS01 & 57.06 & $\mathrm{C}_{4} \mathrm{H}_{9}$ \\
\hline $1112-2$ & $\mathrm{C}_{14} \mathrm{H}_{12}$ or $\mathrm{C}_{12} \mathrm{H}_{8} \mathrm{~N}_{2}$ & SS01 & 180.08 & $\mathrm{C}_{14} \mathrm{H}_{12}$ or $\mathrm{C}_{12} \mathrm{H}_{8} \mathrm{~N}_{2}$ \\
\hline $1112-3$ & $\mathrm{H}$ & SS01 & 1.01 & $\mathrm{H}$ \\
\hline $1112-3$ & $\mathrm{CH}$ & SS01 & 13.01 & $\mathrm{CH}$ \\
\hline $1112-3$ & $\mathrm{O}$ & SS01 & 15.99 & $\mathrm{O}$ \\
\hline $1112-3$ & $\mathrm{OH}$ & SS01 & 17.00 & $\mathrm{OH}$ \\
\hline $1113-1$ & $\mathrm{Na}$ & $\mathrm{SS} 02$ & 22.99 & $\mathrm{Na}$ \\
\hline $1113-1$ & $\mathrm{Al}$ & SS02 & 26.98 & $\mathrm{Al}$ \\
\hline $1113-1$ & $\mathrm{Si}$ & $\mathrm{SS} 02$ & 27.98 & $\mathrm{Si}$ \\
\hline $1113-1$ & $\mathrm{~K}$ & $\mathrm{SS} 02$ & 38.96 & $\mathrm{~K}$ \\
\hline $1114-1$ & $\mathrm{Al}$ & $\mathrm{SS} 03$ & 26.98 & $\mathrm{Al}$ \\
\hline $1114-1$ & $\mathrm{Si}$ & SS03 & 27.98 & $\mathrm{Si}$ \\
\hline $1114-1$ & $\mathrm{~K}$ & SS03 & 38.96 & K \\
\hline $1114-1$ & $\mathrm{C}_{3} \mathrm{H}_{5}$ & SS03 & 41.04 & $\mathrm{C}_{3} \mathrm{H}_{5}$ \\
\hline $1114-1$ & $\mathrm{Fe}$ & SS03 & 55.94 & $\mathrm{Fe}$ \\
\hline $1114-1$ & $\mathrm{C}_{14} \mathrm{H}_{12}$ or $\mathrm{C}_{12} \mathrm{H}_{8} \mathrm{~N}_{2}$ & SS03 & 180.08 & $\mathrm{C}_{14} \mathrm{H}_{12}$ or $\mathrm{C}_{12} \mathrm{H}_{8} \mathrm{~N}_{2}$ \\
\hline $1114-2$ & $\mathrm{Al}$ & SS03 & 26.98 & $\mathrm{Al}$ \\
\hline $1114-2$ & $\mathrm{Si}$ & $\mathrm{SS} 03$ & 27.98 & $\mathrm{Si}$ \\
\hline $1114-2$ & $\mathrm{~K}$ & $\mathrm{SS} 03$ & 38.96 & K \\
\hline $1114-2$ & $\mathrm{C}_{3} \mathrm{H}_{5}$ & $\mathrm{SS} 03$ & 41.04 & $\mathrm{C}_{3} \mathrm{H}_{5}$ \\
\hline $1114-2$ & $\mathrm{C}_{3} \mathrm{H}_{7}$ & SS03 & 43.05 & $\mathrm{C}_{3} \mathrm{H}_{7}$ \\
\hline $1114-2$ & $\mathrm{Fe}$ & SS03 & 55.94 & $\mathrm{Fe}$ \\
\hline $1114-2$ & $\mathrm{C}_{6} \mathrm{H}_{5}$ & SS03 & 77.03 & $\mathrm{C}_{6} \mathrm{H}_{5}$ \\
\hline $1114-2$ & $\mathrm{C}_{14} \mathrm{H}_{12}$ or $\mathrm{C}_{12} \mathrm{H}_{8} \mathrm{~N}_{2}$ & SS03 & 180.08 & $\mathrm{C}_{14} \mathrm{H}_{12}$ or $\mathrm{C}_{12} \mathrm{H}_{8} \mathrm{~N}_{2}$ \\
\hline $1114-3$ & $\mathrm{Al}$ & SS03 & 26.98 & $\mathrm{Al}$ \\
\hline $1114-3$ & $\mathrm{Si}$ & SS03 & 27.98 & $\mathrm{Si}$ \\
\hline $1114-3$ & $\mathrm{C}_{2} \mathrm{H}_{5}$ & SS03 & 29.04 & $\mathrm{C}_{2} \mathrm{H}_{5}$ \\
\hline $1114-3$ & $\mathrm{~K}$ & $\mathrm{SS} 03$ & 38.96 & $\mathrm{~K}$ \\
\hline $1114-3$ & $\mathrm{C}_{3} \mathrm{H}_{5}$ & $\mathrm{SS} 03$ & 41.04 & $\mathrm{C}_{3} \mathrm{H}_{5}$ \\
\hline $1114-3$ & $\mathrm{C}_{3} \mathrm{H}_{7}$ & SS03 & 43.05 & $\mathrm{C}_{3} \mathrm{H}_{7}$ \\
\hline $1114-3$ & $\mathrm{C}_{4} \mathrm{H}_{7}$ & SS03 & 55.05 & $\mathrm{C}_{4} \mathrm{H}_{7}$ \\
\hline $1114-3$ & $\mathrm{Fe}$ & SS03 & 55.94 & $\mathrm{Fe}$ \\
\hline $1114-3$ & $\mathrm{C}_{4} \mathrm{H}_{9}$ & SS03 & 57.07 & $\mathrm{C}_{4} \mathrm{H}_{9}$ \\
\hline $1114-3$ & $\mathrm{C}_{6} \mathrm{H}_{5}$ & SS03 & 77.03 & $\mathrm{C}_{6} \mathrm{H}_{5}$ \\
\hline
\end{tabular}




\begin{tabular}{|c|c|c|c|c|}
\hline \multicolumn{5}{|c|}{ SPECTRAL FEATURES TABLE (CONT.) } \\
\hline Spectrum ID \# & Species & Source & Mass & Label \\
\hline $1114-3$ & $\mathrm{C}_{14} \mathrm{H}_{12}$ or $\mathrm{C}_{12} \mathrm{H}_{8} \mathrm{~N}_{2}$ & SS03 & 180.08 & $\mathrm{C}_{14} \mathrm{H}_{12}$ or $\mathrm{C}_{12} \mathrm{H}_{8} \mathrm{~N}_{2}$ \\
\hline $1115-1$ & $\mathrm{Na}$ & SS04 & 22.99 & $\mathrm{Na}$ \\
\hline $1115-1$ & $\mathrm{Al}$ & SS04 & 26.98 & $\mathrm{Al}$ \\
\hline 1115-1 & $\mathrm{Si}$ & SS04 & 27.98 & $\mathrm{Si}$ \\
\hline $1115-1$ & K & SS04 & 38.96 & $\mathrm{~K}$ \\
\hline $1115-1$ & $\mathrm{Fe}$ & SS04 & 55.94 & $\mathrm{Fe}$ \\
\hline 1116-1 & $\mathrm{Al}$ & SS05 & 26.98 & $\mathrm{Al}$ \\
\hline 1116-1 & $\mathrm{Si}$ & SS05 & 27.98 & $\mathrm{Si}$ \\
\hline 1116-1 & $\mathrm{C}_{2} \mathrm{H}_{5}$ & SS05 & 29.04 & $\mathrm{C}_{2} \mathrm{H}_{5}$ \\
\hline 1116-1 & $\mathrm{K}$ & SS05 & 38.96 & $\mathrm{~K}$ \\
\hline 1116-1 & $\mathrm{C}_{3} \mathrm{H}_{5}$ & SS05 & 41.04 & $\mathrm{C}_{3} \mathrm{H}_{5}$ \\
\hline 1116-1 & $\mathrm{C}_{3} \mathrm{H}_{7}$ & SS05 & 43.05 & $\mathrm{C}_{3} \mathrm{H}_{7}$ \\
\hline 1116-1 & $\mathrm{Fe}$ & SS05 & 55.94 & $\mathrm{Fe}$ \\
\hline $1116-1$ & $\mathrm{C}_{4} \mathrm{H}_{9}$ & SS05 & 57.07 & $\mathrm{C}_{4} \mathrm{H}_{9}$ \\
\hline 1116-1 & $\mathrm{C}_{6} \mathrm{H}_{5}$ & SS05 & 77.03 & $\mathrm{C}_{6} \mathrm{H}_{5}$ \\
\hline $1116-1$ & $\mathrm{C}_{14} \mathrm{H}_{12}$ or $\mathrm{C}_{12} \mathrm{H}_{8} \mathrm{~N}_{2}$ & SS05 & 180.08 & $\mathrm{C}_{14} \mathrm{H}_{12}$ or $\mathrm{C}_{12} \mathrm{H}_{8} \mathrm{~N}_{2}$ \\
\hline $1116-2$ & $\mathrm{Al}$ & SS05 & 26.98 & $\mathrm{Al}$ \\
\hline $1116-2$ & $\mathrm{Si}$ & SS05 & 27.98 & $\mathrm{Si}$ \\
\hline $1116-2$ & $\mathrm{C}_{2} \mathrm{H}_{5}$ & SS05 & 29.04 & $\mathrm{C}_{2} \mathrm{H}_{5}$ \\
\hline $1116-2$ & $\mathrm{~K}$ & SS05 & 38.96 & $\mathrm{~K}$ \\
\hline $1116-2$ & $\mathrm{C}_{3} \mathrm{H}_{5}$ & SS05 & 41.04 & $\mathrm{C}_{3} \mathrm{H}_{5}$ \\
\hline $1116-2$ & $\mathrm{Fe}$ & SS05 & 55.94 & $\mathrm{Fe}$ \\
\hline $1116-2$ & $\mathrm{C}_{6} \mathrm{H}_{5}$ & SS05 & 77.03 & $\mathrm{C}_{6} \mathrm{H}_{5}$ \\
\hline $1116-2$ & $\mathrm{C}_{14} \mathrm{H}_{12}$ or $\mathrm{C}_{12} \mathrm{H}_{8} \mathrm{~N}_{2}$ & SS05 & 180.08 & $\mathrm{C}_{14} \mathrm{H}_{12}$ or $\mathrm{C}_{12} \mathrm{H}_{8} \mathrm{~N}_{2}$ \\
\hline 1117-1 & $\mathrm{Al}$ & SS06 & 26.98 & $\mathrm{Al}$ \\
\hline $1117-1$ & $\mathrm{Si}$ & SS06 & 27.98 & $\mathrm{Si}$ \\
\hline 1117-1 & $\mathrm{C}_{2} \mathrm{H}_{5}$ & SS06 & 29.04 & $\mathrm{C}_{2} \mathrm{H}_{5}$ \\
\hline $1117-1$ & $\mathrm{~K}$ & SS06 & 38.96 & $\mathrm{~K}$ \\
\hline 1117-1 & $\mathrm{Fe}$ & SS06 & 55.94 & $\mathrm{Fe}$ \\
\hline 1117-1 & $\mathrm{C}_{4} \mathrm{H}_{12} \mathrm{~N}$ & SS06 & 74.10 & $\mathrm{C}_{4} \mathrm{H}_{12} \mathrm{~N}$ \\
\hline $1117-1$ & $\mathrm{C}_{14} \mathrm{H}_{12}$ or $\mathrm{C}_{12} \mathrm{H}_{8} \mathrm{~N}_{2}$ & SS06 & 180.08 & $\mathrm{C}_{14} \mathrm{H}_{12}$ or $\mathrm{C}_{12} \mathrm{H}_{8} \mathrm{~N}_{2}$ \\
\hline $1118-1$ & $\mathrm{Na}$ & SS07 & 22.99 & $\mathrm{Na}$ \\
\hline 1118-1 & $\mathrm{Al}$ & SS07 & 26.98 & $\mathrm{Al}$ \\
\hline 1118-1 & $\mathrm{Si}$ & SS07 & 27.98 & $\mathrm{Si}$ \\
\hline $1118-1$ & $\mathrm{~K}$ & SS07 & 38.96 & $\mathrm{~K}$ \\
\hline 1118-1 & $\mathrm{C}_{3} \mathrm{H}_{5}$ & SS07 & 41.04 & $\mathrm{C}_{3} \mathrm{H}_{5}$ \\
\hline 1118-1 & $\mathrm{C}_{3} \mathrm{H}_{7}$ & SS07 & 43.05 & $\mathrm{C}_{3} \mathrm{H}_{7}$ \\
\hline 1118-1 & $\mathrm{C}_{4} \mathrm{H}_{7}$ & SS07 & 55.05 & $\mathrm{C}_{4} \mathrm{H}_{7}$ \\
\hline $1118-1$ & $\mathrm{Fe}$ & SS07 & 55.94 & $\mathrm{Fe}$ \\
\hline $1118-1$ & $\mathrm{C}_{14} \mathrm{H}_{12}$ or $\mathrm{C}_{12} \mathrm{H}_{8} \mathrm{~N}_{2}$ & SS07 & 180.08 & $\mathrm{C}_{14} \mathrm{H}_{12}$ or $\mathrm{C}_{12} \mathrm{H}_{8} \mathrm{~N}_{2}$ \\
\hline $1118-2$ & $\mathrm{Na}$ & SS07 & 22.99 & $\mathrm{Na}$ \\
\hline $1118-2$ & $\mathrm{Al}$ & SS07 & 26.98 & $\mathrm{Al}$ \\
\hline $1118-2$ & $\mathrm{Si}$ & SS07 & 27.98 & $\mathrm{Si}$ \\
\hline $1118-2$ & $\mathrm{~K}$ & SS07 & 38.96 & $\mathrm{~K}$ \\
\hline $1118-2$ & $\mathrm{Fe}$ & SS07 & 55.94 & $\mathrm{Fe}$ \\
\hline
\end{tabular}




\begin{tabular}{|c|c|c|c|c|}
\hline \multicolumn{5}{|c|}{ SPECTRAL FEATURES TABLE (CONT.) } \\
\hline Spectrum ID \# & Species & Source & Mass & Labe \\
\hline $1119-1$ & $\mathrm{Na}$ & SS08 & 22.99 & $\mathrm{Na}$ \\
\hline $1119-1$ & $\mathrm{Al}$ & SS08 & 26.98 & $\mathrm{Al}$ \\
\hline $1119-1$ & $\mathrm{Si}$ & SS08 & 27.98 & $\mathrm{Si}$ \\
\hline $1119-1$ & $\mathrm{~K}$ & SS08 & 38.96 & $\mathrm{~K}$ \\
\hline $1119-1$ & $\mathrm{Fe}$ & SS08 & 55.94 & $\mathrm{Fe}$ \\
\hline $1119-1$ & $\mathrm{C}_{6} \mathrm{H}_{5}$ & SS08 & 77.03 & $\mathrm{C}_{6} \mathrm{H}_{5}$ \\
\hline $1120-1$ & $\mathrm{Na}$ & SS09 & 22.99 & $\mathrm{Na}$ \\
\hline $1120-1$ & $\mathrm{Al}$ & SS09 & 26.98 & $\mathrm{Al}$ \\
\hline $1120-1$ & $\mathrm{Si}$ & SS09 & 27.98 & $\mathrm{Si}$ \\
\hline $1120-1$ & $\mathrm{~K}$ & SS09 & 38.96 & $\mathrm{~K}$ \\
\hline $1121-1$ & $\mathrm{Na}$ & SS10 & 22.99 & $\mathrm{Na}$ \\
\hline $1121-1$ & $\mathrm{Al}$ & SS10 & 26.98 & $\mathrm{Al}$ \\
\hline $1121-1$ & $\mathrm{Si}$ & SS10 & 27.98 & $\mathrm{Si}$ \\
\hline $1121-1$ & $\mathrm{~K}$ & SS10 & 38.96 & $\mathrm{~K}$ \\
\hline $1121-1$ & $\mathrm{Fe}$ & SS10 & 55.94 & $\mathrm{Fe}$ \\
\hline $1121-2$ & $\mathrm{Na}$ & SS10 & 22.99 & $\mathrm{Na}$ \\
\hline $1121-2$ & $\mathrm{Al}$ & SS10 & 26.98 & $\mathrm{Al}$ \\
\hline $1121-2$ & $\mathrm{Si}$ & SS10 & 27.98 & $\mathrm{Si}$ \\
\hline $1121-2$ & $\mathrm{~K}$ & SS10 & 38.96 & $\mathrm{~K}$ \\
\hline $1121-2$ & $\mathrm{Fe}$ & SS10 & 55.94 & $\mathrm{Fe}$ \\
\hline $1122-1$ & $\mathrm{C}_{2} \mathrm{H}_{3}$ & SS11 & 27.02 & $\mathrm{C}_{2} \mathrm{H}_{3}$ \\
\hline $1122-1$ & $\mathrm{C}_{3} \mathrm{H}_{5}$ & SS11 & 41.04 & $\mathrm{C}_{3} \mathrm{H}_{5}$ \\
\hline $1122-1$ & $\mathrm{C}_{3} \mathrm{H}_{7}$ & SS11 & 43.05 & $\mathrm{C}_{3} \mathrm{H}_{7}$ \\
\hline $1122-1$ & $\mathrm{C}_{4} \mathrm{H}_{9}$ & SS11 & 57.07 & $\mathrm{C}_{4} \mathrm{H}_{9}$ \\
\hline $1122-2$ & $\mathrm{C}_{2} \mathrm{H}_{3}$ & SS11 & 27.02 & $\mathrm{C}_{2} \mathrm{H}_{3}$ \\
\hline $1122-2$ & $\mathrm{C}_{3} \mathrm{H}_{5}$ & SS11 & 41.04 & $\mathrm{C}_{3} \mathrm{H}_{5}$ \\
\hline $1122-2$ & $\mathrm{C}_{3} \mathrm{H}_{7}$ & SS11 & 43.05 & $\mathrm{C}_{3} \mathrm{H}_{7}$ \\
\hline $1122-2$ & $\mathrm{C}_{4} \mathrm{H}_{9}$ & SS11 & 57.07 & $\mathrm{C}_{4} \mathrm{H}_{9}$ \\
\hline $1122-2$ & $\mathrm{C}_{6} \mathrm{H}_{5}$ & SS11 & 77.03 & $\mathrm{C}_{6} \mathrm{H}_{5}$ \\
\hline $1122-3$ & $\mathrm{Na}$ & SS11 & 22.99 & $\mathrm{Na}$ \\
\hline $1122-3$ & $\mathrm{Al}$ & SS11 & 26.98 & $\mathrm{Al}$ \\
\hline $1122-3$ & $\mathrm{Si}$ & SS11 & 27.98 & $\mathrm{Si}$ \\
\hline $1122-3$ & $\mathrm{~K}$ & SS11 & 38.96 & $\mathrm{~K}$ \\
\hline $1123-1$ & $\mathrm{Na}$ & SS12 & 22.99 & $\mathrm{Na}$ \\
\hline $1123-1$ & $\mathrm{Al}$ & SS12 & 26.98 & $\mathrm{Al}$ \\
\hline $1123-1$ & $\mathrm{Si}$ & SS12 & 27.98 & $\mathrm{Si}$ \\
\hline $1123-1$ & $\mathrm{~K}$ & SS12 & 38.96 & $\mathrm{~K}$ \\
\hline $1124-1$ & $\mathrm{C}_{2} \mathrm{H}_{3}$ & SS13 & 27.03 & $\mathrm{C}_{2} \mathrm{H}_{3}$ \\
\hline $1124-1$ & $\mathrm{C}_{3} \mathrm{H}_{5}$ & SS13 & 41.04 & $\mathrm{C}_{3} \mathrm{H}_{5}$ \\
\hline $1124-1$ & $\mathrm{C}_{3} \mathrm{H}_{7}$ & SS13 & 43.05 & $\mathrm{C}_{3} \mathrm{H}_{7}$ \\
\hline $1124-1$ & $\mathrm{C}_{4} \mathrm{H}_{9}$ & SS13 & 57.07 & $\mathrm{C}_{4} \mathrm{H}_{9}$ \\
\hline $1125-1$ & $\mathrm{Na}$ & SS14 & 22.99 & $\mathrm{Na}$ \\
\hline $1125-1$ & $\mathrm{C}_{2} \mathrm{H}_{3}$ & SS14 & 27.03 & $\mathrm{C}_{2} \mathrm{H}_{3}$ \\
\hline $1125-1$ & $\mathrm{C}_{3} \mathrm{H}_{5}$ & SS14 & 41.04 & $\mathrm{C}_{3} \mathrm{H}_{5}$ \\
\hline $1125-1$ & $\mathrm{C}_{3} \mathrm{H}_{7}$ & SS14 & 43.05 & $\mathrm{C}_{3} \mathrm{H}_{7}$ \\
\hline
\end{tabular}




\begin{tabular}{|c|c|c|c|c|}
\hline \multicolumn{5}{|c|}{ SPECTRAL FEATURES TABLE (CONT.) } \\
\hline Spectrum ID \# & Species & Source & Mass & Label \\
\hline $1125-1$ & $\mathrm{C}_{4} \mathrm{H}_{9}$ & SS14 & 57.07 & $\mathrm{C}_{4} \mathrm{H}_{9}$ \\
\hline $1125-2$ & $\mathrm{C}_{2} \mathrm{H}_{3}$ & SS14 & 27.03 & $\mathrm{C}_{2} \mathrm{H}_{3}$ \\
\hline $1125-2$ & $\mathrm{C}_{3} \mathrm{H}_{5}$ & SS14 & 41.04 & $\mathrm{C}_{3} \mathrm{H}_{5}$ \\
\hline $1125-2$ & $\mathrm{C}_{3} \mathrm{H}_{7}$ & SS14 & 43.05 & $\mathrm{C}_{3} \mathrm{H}_{7}$ \\
\hline $1125-2$ & $\mathrm{C}_{4} \mathrm{H}_{9}$ & SS14 & 57.07 & $\mathrm{C}_{4} \mathrm{H}_{9}$ \\
\hline $1126-1$ & $\mathrm{C}_{2} \mathrm{H}_{5}$ & SS15 & 29.04 & $\mathrm{C}_{2} \mathrm{H}_{5}$ \\
\hline $1126-1$ & $\mathrm{C}_{3} \mathrm{H}_{5}$ & SS15 & 41.04 & $\mathrm{C}_{3} \mathrm{H}_{5}$ \\
\hline $1126-1$ & $\mathrm{C}_{3} \mathrm{H}_{7}$ & SS15 & 43.05 & $\mathrm{C}_{3} \mathrm{H}_{7}$ \\
\hline $1126-1$ & $\mathrm{C}_{4} \mathrm{H}_{9}$ & SS15 & 57.07 & $\mathrm{C}_{4} \mathrm{H}_{9}$ \\
\hline $1127-1$ & $\mathrm{Na}$ & SS16 & 22.99 & $\mathrm{Na}$ \\
\hline $1127-1$ & $\mathrm{Al}$ & SS16 & 26.98 & $\mathrm{Al}$ \\
\hline $1127-1$ & $\mathrm{Si}$ & SS16 & 27.98 & $\mathrm{Si}$ \\
\hline $1127-1$ & $\mathrm{~K}$ & SS16 & 38.96 & K \\
\hline $1127-1$ & $\mathrm{C}_{14} \mathrm{H}_{12}$ or $\mathrm{C}_{12} \mathrm{H}_{8} \mathrm{~N}_{2}$ & SS16 & 180.08 & $\mathrm{C}_{14} \mathrm{H}_{12}$ or $\mathrm{C}_{12} \mathrm{H}_{8} \mathrm{~N}_{2}$ \\
\hline $1128-1$ & $\mathrm{Na}$ & SS17 & 22.99 & $\mathrm{Na}$ \\
\hline $1128-1$ & $\mathrm{Al}$ & SS17 & 26.98 & $\mathrm{Al}$ \\
\hline $1128-1$ & $\mathrm{Si}$ & SS17 & 27.98 & $\mathrm{Si}$ \\
\hline $1128-1$ & $\mathrm{~K}$ & SS17 & 38.96 & K \\
\hline $1128-1$ & $\mathrm{C}_{14} \mathrm{H}_{12}$ or $\mathrm{C}_{12} \mathrm{H}_{8} \mathrm{~N}_{2}$ & SS17 & 180.08 & $\mathrm{C}_{14} \mathrm{H}_{12}$ or $\mathrm{C}_{12} \mathrm{H}_{8} \mathrm{~N}_{2}$ \\
\hline $1129-1$ & $\mathrm{Na}$ & SS18 & 22.99 & $\mathrm{Na}$ \\
\hline $1129-1$ & $\mathrm{Al}$ & SS18 & 26.98 & $\mathrm{Al}$ \\
\hline $1129-1$ & $\mathrm{Si}$ & SS18 & 27.98 & $\mathrm{Si}$ \\
\hline $1129-1$ & $\mathrm{~K}$ & SS18 & 38.96 & $\mathrm{~K}$ \\
\hline $1129-1$ & $\mathrm{C}_{14} \mathrm{H}_{12}$ or $\mathrm{C}_{12} \mathrm{H}_{8} \mathrm{~N}_{2}$ & SS18 & 180.08 & $\mathrm{C}_{14} \mathrm{H}_{12}$ or $\mathrm{C}_{12} \mathrm{H}_{8} \mathrm{~N}_{2}$ \\
\hline $1129-2$ & $\mathrm{Na}$ & SS18 & 22.99 & $\mathrm{Na}$ \\
\hline $1129-2$ & $\mathrm{Al}$ & SS18 & 26.98 & $\mathrm{Al}$ \\
\hline $1129-2$ & $\mathrm{~K}$ & SS18 & 38.96 & K \\
\hline $1129-2$ & $\mathrm{C}_{3} \mathrm{H}_{5}$ & SS18 & 41.04 & $\mathrm{C}_{3} \mathrm{H}_{5}$ \\
\hline $1129-2$ & $\mathrm{C}_{4} \mathrm{H}_{9}$ & SS18 & 57.07 & $\mathrm{C}_{4} \mathrm{H}_{9}$ \\
\hline $1129-2$ & $\mathrm{C}_{14} \mathrm{H}_{12}$ or $\mathrm{C}_{12} \mathrm{H}_{8} \mathrm{~N}_{2}$ & SS18 & 180.08 & $\mathrm{C}_{14} \mathrm{H}_{12}$ or $\mathrm{C}_{12} \mathrm{H}_{8} \mathrm{~N}_{2}$ \\
\hline 1130-1 & $\mathrm{Na}$ & SS19 & 22.99 & $\mathrm{Na}$ \\
\hline 1130-1 & $\mathrm{Al}$ & SS19 & 26.98 & $\mathrm{Al}$ \\
\hline $1130-1$ & $\mathrm{Si}$ & SS19 & 27.98 & $\mathrm{Si}$ \\
\hline 1130-1 & $\mathrm{K}$ & SS19 & 38.96 & $\mathrm{~K}$ \\
\hline $1130-1$ & $\mathrm{C}_{14} \mathrm{H}_{12}$ or $\mathrm{C}_{12} \mathrm{H}_{8} \mathrm{~N}_{2}$ & SS19 & 180.08 & $\mathrm{C}_{14} \mathrm{H}_{12}$ or $\mathrm{C}_{12} \mathrm{H}_{8} \mathrm{~N}_{2}$ \\
\hline $1131-1$ & $\mathrm{Na}$ & blackthunder & 22.99 & $\mathrm{Na}$ \\
\hline $1131-1$ & $\mathrm{Al}$ & blackthunder & 26.98 & $\mathrm{Al}$ \\
\hline $1131-1$ & $\mathrm{Si}$ & blackthunder & 27.98 & $\mathrm{Si}$ \\
\hline $1131-1$ & $\mathrm{~K}$ & blackthunder & 38.96 & $\mathrm{~K}$ \\
\hline $1131-1$ & $\mathrm{Fe}$ & blackthunder & 55.94 & $\mathrm{Fe}$ \\
\hline $1132-1$ & $\mathrm{Na}$ & CJR012 & 22.99 & $\mathrm{Na}$ \\
\hline $1132-1$ & $\mathrm{Al}$ & CJR012 & 26.98 & $\mathrm{Al}$ \\
\hline $1132-1$ & $\mathrm{Si}$ & CJR012 & 27.98 & $\mathrm{Si}$ \\
\hline $1132-1$ & $\mathrm{~K}$ & CJR012 & 38.96 & $\mathrm{~K}$ \\
\hline $1132-1$ & $\mathrm{Fe}$ & CJR012 & 55.94 & $\mathrm{Fe}$ \\
\hline
\end{tabular}




\begin{tabular}{|c|c|c|c|c|}
\hline \multicolumn{5}{|c|}{ SPECTRAL FEATURES TABLE (CONT.) } \\
\hline Spectrum ID \# & Species & Source & Mass & Label \\
\hline $1133-1$ & $\mathrm{Al}$ & GAT034 & 26.98 & $\mathrm{Al}$ \\
\hline $1133-1$ & $\mathrm{Si}$ & GAT034 & 27.98 & $\mathrm{Si}$ \\
\hline $1133-1$ & $\mathrm{~K}$ & GAT034 & 38.96 & $\mathrm{~K}$ \\
\hline $1133-1$ & $\mathrm{Fe}$ & GAT034 & 55.94 & $\mathrm{Fe}$ \\
\hline $1133-2$ & $\mathrm{Na}$ & GAT034 & 22.99 & $\mathrm{Na}$ \\
\hline $1133-2$ & $\mathrm{Al}$ & GAT034 & 26.98 & $\mathrm{Al}$ \\
\hline $1133-2$ & $\mathrm{Si}$ & GAT034 & 27.98 & $\mathrm{Si}$ \\
\hline $1133-2$ & $\mathrm{~K}$ & GAT034 & 38.96 & $\mathrm{~K}$ \\
\hline $1133-2$ & $\mathrm{Fe}$ & GAT034 & 55.94 & $\mathrm{Fe}$ \\
\hline $1134-1$ & $\mathrm{Al}$ & GAT035 & 26.98 & $\mathrm{Al}$ \\
\hline 1134-1 & $\mathrm{Si}$ & GAT035 & 27.98 & $\mathrm{Si}$ \\
\hline 1134-1 & $\mathrm{K}$ & GAT035 & 38.96 & $\mathrm{~K}$ \\
\hline $1134-2$ & $\mathrm{Na}$ & GAT035 & 22.99 & $\mathrm{Na}$ \\
\hline $1134-2$ & $\mathrm{Al}$ & GAT035 & 26.98 & $\mathrm{Al}$ \\
\hline $1134-2$ & $\mathrm{Si}$ & GAT035 & 27.98 & $\mathrm{Si}$ \\
\hline $1134-2$ & K & GAT035 & 38.96 & K \\
\hline $1135-1$ & $\mathrm{Na}$ & GBJ010 & 22.99 & $\mathrm{Na}$ \\
\hline $1135-1$ & $\mathrm{Al}$ & GBJ010 & 26.98 & $\mathrm{Al}$ \\
\hline $1135-1$ & $\mathrm{Si}$ & GBJ010 & 27.98 & $\mathrm{Si}$ \\
\hline $1135-1$ & K & GBJ010 & 38.96 & $\mathrm{~K}$ \\
\hline 1136-1 & $\mathrm{Na}$ & GLJ005 & 22.99 & $\mathrm{Na}$ \\
\hline $1136-1$ & $\mathrm{Al}$ & GLJ005 & 26.98 & $\mathrm{Al}$ \\
\hline $1136-1$ & $\mathrm{Si}$ & GLJ005 & 27.98 & $\mathrm{Si}$ \\
\hline $1136-1$ & K & GLJ005 & 38.96 & K \\
\hline $1136-1$ & $\mathrm{Fe}$ & GLJ005 & 55.94 & $\mathrm{Fe}$ \\
\hline $1136-2$ & $\mathrm{Na}$ & GLJ005 & 22.99 & $\mathrm{Na}$ \\
\hline $1136-2$ & $\mathrm{Al}$ & GLJ005 & 26.98 & $\mathrm{Al}$ \\
\hline $1136-2$ & $\mathrm{Si}$ & GLJ005 & 27.98 & $\mathrm{Si}$ \\
\hline $1136-2$ & K & GLJ005 & 38.96 & K \\
\hline $1136-2$ & $\mathrm{Fe}$ & GLJ005 & 55.94 & $\mathrm{Fe}$ \\
\hline $1137-1$ & $\mathrm{Al}$ & GPB062 & 26.98 & $\mathrm{Al}$ \\
\hline $1137-1$ & $\mathrm{Si}$ & GPB062 & 27.98 & $\mathrm{Si}$ \\
\hline $1137-1$ & K & GPB062 & 38.96 & $\mathrm{~K}$ \\
\hline $1137-1$ & $\mathrm{Fe}$ & GPB062 & 55.94 & $\mathrm{Fe}$ \\
\hline $1137-2$ & $\mathrm{Al}$ & GPB062 & 26.98 & $\mathrm{Al}$ \\
\hline $1137-2$ & $\mathrm{Si}$ & GPB062 & 27.98 & $\mathrm{Si}$ \\
\hline $1137-2$ & $\mathrm{C}_{2} \mathrm{H}_{5}$ & GPB062 & 29.04 & $\mathrm{C}_{2} \mathrm{H}_{5}$ \\
\hline $1137-2$ & K & GPB062 & 38.96 & $\mathrm{~K}$ \\
\hline $1137-2$ & $\mathrm{C}_{3} \mathrm{H}_{5}$ & GPB062 & 41.04 & $\mathrm{C}_{3} \mathrm{H}_{5}$ \\
\hline $1137-2$ & $\mathrm{Fe}$ & GPB062 & 55.94 & $\mathrm{Fe}$ \\
\hline $1138-1$ & $\mathrm{Al}$ & HGB011 & 26.98 & $\mathrm{Al}$ \\
\hline $1138-1$ & $\mathrm{Si}$ & HGB011 & 27.98 & $\mathrm{Si}$ \\
\hline $1138-1$ & K & HGB011 & 38.96 & K \\
\hline $1138-1$ & $\mathrm{Fe}$ & HGB011 & 55.94 & $\mathrm{Fe}$ \\
\hline $1139-1$ & $\mathrm{Al}$ & HNA015 & 26.98 & $\mathrm{Al}$ \\
\hline
\end{tabular}




\begin{tabular}{|c|c|c|c|c|}
\hline \multicolumn{5}{|c|}{ SPECTRAL FEATURES TABLE (CONT.) } \\
\hline Spectrum ID \# & Species & Source & Mass & Label \\
\hline 1139-1 & $\mathrm{Si}$ & HNA015 & 27.98 & $\mathrm{Si}$ \\
\hline $1139-1$ & $\mathrm{~K}$ & HNA015 & 38.96 & $\mathrm{~K}$ \\
\hline 1139-1 & $\mathrm{Fe}$ & HNA015 & 55.94 & $\mathrm{Fe}$ \\
\hline 1140-1 & $\mathrm{Na}$ & ILL6 & 22.99 & $\mathrm{Na}$ \\
\hline 1140-1 & $\mathrm{Al}$ & ILL6 & 26.98 & $\mathrm{Al}$ \\
\hline 1140-1 & $\mathrm{Si}$ & ILL6 & 27.98 & $\mathrm{Si}$ \\
\hline 1140-1 & $\mathrm{K}$ & ILL6 & 38.96 & $\mathrm{~K}$ \\
\hline 1140-1 & $\mathrm{Fe}$ & ILL6 & 55.94 & $\mathrm{Fe}$ \\
\hline $1140-2$ & $\mathrm{Na}$ & ILL6 & 22.99 & $\mathrm{Na}$ \\
\hline $1140-2$ & $\mathrm{Al}$ & ILL6 & 26.98 & $\mathrm{Al}$ \\
\hline $1140-2$ & $\mathrm{Si}$ & ILL6 & 27.98 & $\mathrm{Si}$ \\
\hline $1140-2$ & $\mathrm{~K}$ & ILL6 & 38.96 & $\mathrm{~K}$ \\
\hline $1140-2$ & $\mathrm{Fe}$ & ILL6 & 55.94 & $\mathrm{Fe}$ \\
\hline $1141-1$ & $\mathrm{Na}$ & JIG014 & 22.99 & $\mathrm{Na}$ \\
\hline $1141-1$ & $\mathrm{Al}$ & JIG014 & 26.98 & $\mathrm{Al}$ \\
\hline $1141-1$ & $\mathrm{Si}$ & JIG014 & 27.98 & $\mathrm{Si}$ \\
\hline 1141-1 & $\mathrm{K}$ & JIG014 & 38.96 & $\mathrm{~K}$ \\
\hline $1141-1$ & $\mathrm{Fe}$ & JIG014 & 55.94 & $\mathrm{Fe}$ \\
\hline $1141-2$ & $\mathrm{Na}$ & JIG014 & 22.99 & $\mathrm{Na}$ \\
\hline $1141-2$ & $\mathrm{Al}$ & JIG014 & 26.98 & $\mathrm{Al}$ \\
\hline $1141-2$ & $\mathrm{Si}$ & JIG014 & 27.98 & $\mathrm{Si}$ \\
\hline $1141-2$ & $\mathrm{~K}$ & JIG014 & 38.96 & $\mathrm{~K}$ \\
\hline $1141-2$ & $\mathrm{Fe}$ & JIG014 & 55.94 & $\mathrm{Fe}$ \\
\hline $1142-1$ & $\mathrm{Na}$ & JIG017 & 22.99 & $\mathrm{Na}$ \\
\hline $1142-1$ & $\mathrm{Al}$ & JIG017 & 26.98 & $\mathrm{Al}$ \\
\hline $1142-1$ & $\mathrm{Si}$ & JIG017 & 27.98 & $\mathrm{Si}$ \\
\hline $1142-1$ & $\mathrm{~K}$ & JIG017 & 38.96 & $\mathrm{~K}$ \\
\hline $1142-1$ & $\mathrm{Fe}$ & JIG017 & 55.94 & $\mathrm{Fe}$ \\
\hline 1143-1 & $\mathrm{Al}$ & РСТ010 & 26.98 & $\mathrm{Al}$ \\
\hline 1143-1 & $\mathrm{Si}$ & РСТ010 & 27.98 & $\mathrm{Si}$ \\
\hline 1143-1 & $\mathrm{K}$ & РСТ010 & 38.96 & $\mathrm{~K}$ \\
\hline 1143-1 & $\mathrm{Fe}$ & РСТ010 & 55.94 & $\mathrm{Fe}$ \\
\hline $1143-2$ & $\mathrm{Al}$ & РСТ010 & 26.98 & $\mathrm{Al}, \mathrm{C}_{2} \mathrm{H}_{3}$ \\
\hline $1143-2$ & $\mathrm{C}_{2} \mathrm{H}_{3}$ & РСТ010 & 27.03 & $\mathrm{Al}, \mathrm{C}_{2} \mathrm{H}_{3}$ \\
\hline $1143-2$ & $\mathrm{Si}$ & РСТ010 & 27.98 & $\mathrm{Si}$ \\
\hline $1143-2$ & $\mathrm{C}_{2} \mathrm{H}_{5}$ & РCT010 & 29.04 & $\mathrm{C}_{2} \mathrm{H}_{5}$ \\
\hline $1143-2$ & $\mathrm{~K}$ & РСТ010 & 38.96 & $\mathrm{~K}$ \\
\hline $1143-2$ & $\mathrm{C}_{3} \mathrm{H}_{5}$ & РСТ010 & 41.04 & $\mathrm{C}_{3} \mathrm{H}_{5}$ \\
\hline $1143-2$ & $\mathrm{C}_{4} \mathrm{H}_{7}$ & РСТ010 & 55.05 & $\mathrm{C}_{4} \mathrm{H}_{7}$ \\
\hline 1144-1 & $\mathrm{Al}$ & РCT012 & 26.98 & $\mathrm{Al}$ \\
\hline 1144-1 & $\mathrm{Si}$ & РСТ012 & 27.98 & $\mathrm{Si}$ \\
\hline $1144-1$ & $\mathrm{~K}$ & РСТ012 & 38.96 & $\mathrm{~K}$ \\
\hline 1144-1 & $\mathrm{Fe}$ & РСТ012 & 55.94 & $\mathrm{Fe}$ \\
\hline $1144-2$ & $\mathrm{Na}$ & РCT012 & 22.99 & $\mathrm{Na}$ \\
\hline $1144-2$ & $\mathrm{Al}$ & РСТ012 & 26.98 & $\mathrm{Al}$ \\
\hline
\end{tabular}




\begin{tabular}{|c|c|c|c|c|}
\hline \multicolumn{5}{|c|}{ SPECTRAL FEATURES TABLE (CONT.) } \\
\hline Spectrum ID \# & Species & Source & Mass & Labe \\
\hline $1144-2$ & $\mathrm{Si}$ & РCT012 & 27.98 & $\mathrm{Si}$ \\
\hline $1144-2$ & $\mathrm{~K}$ & РCT012 & 38.96 & $\mathrm{~K}$ \\
\hline $1144-2$ & $\mathrm{Fe}$ & РCT012 & 55.94 & $\mathrm{Fe}$ \\
\hline $1145-1$ & $\mathrm{Na}$ & RBL014 & 22.99 & $\mathrm{Na}$ \\
\hline $1145-1$ & $\mathrm{Al}$ & RBL014 & 26.98 & $\mathrm{Al}$ \\
\hline $1145-1$ & $\mathrm{Si}$ & RBL014 & 27.98 & $\mathrm{Si}$ \\
\hline $1145-1$ & $\mathrm{~K}$ & RBL014 & 38.96 & $\mathrm{~K}$ \\
\hline $1145-1$ & $\mathrm{Fe}$ & RBL014 & 55.94 & $\mathrm{Fe}$ \\
\hline $1146-1$ & $\mathrm{Na}$ & RLL012 & 22.99 & $\mathrm{Na}$ \\
\hline $1146-1$ & $\mathrm{Al}$ & RLL012 & 26.98 & $\mathrm{Al}$ \\
\hline $1146-1$ & $\mathrm{Si}$ & RLL012 & 27.98 & $\mathrm{Si}$ \\
\hline $1146-1$ & K & RLL012 & 38.96 & $\mathrm{~K}$ \\
\hline $1146-1$ & $\mathrm{Fe}$ & RLL012 & 55.94 & $\mathrm{Fe}$ \\
\hline $1146-2$ & $\mathrm{Na}$ & RLL012 & 22.99 & $\mathrm{Na}$ \\
\hline $1146-2$ & $\mathrm{Al}$ & RLL012 & 26.98 & $\mathrm{Al}$ \\
\hline $1146-2$ & $\mathrm{Si}$ & RLL012 & 27.98 & $\mathrm{Si}$ \\
\hline $1146-2$ & K & RLL012 & 38.96 & K \\
\hline $1146-2$ & $\mathrm{Fe}$ & RLL012 & 55.94 & $\mathrm{Fe}$ \\
\hline $1147-1$ & $\mathrm{Na}$ & RLL014 & 22.99 & $\mathrm{Na}$ \\
\hline $1147-1$ & $\mathrm{Al}$ & RLL014 & 26.98 & $\mathrm{Al}$ \\
\hline $1147-1$ & $\mathrm{Si}$ & RLL014 & 27.98 & $\mathrm{Si}$ \\
\hline $1147-1$ & K & RLL014 & 38.96 & K \\
\hline $1147-1$ & $\mathrm{Fe}$ & RLL014 & 55.94 & $\mathrm{Fe}$ \\
\hline $1147-2$ & $\mathrm{Na}$ & RLL014 & 22.99 & $\mathrm{Na}$ \\
\hline $1147-2$ & $\mathrm{Al}$ & RLL014 & 26.98 & $\mathrm{Al}$ \\
\hline $1147-2$ & $\mathrm{Si}$ & RLL014 & 27.98 & $\mathrm{Si}$ \\
\hline $1147-2$ & K & RLL014 & 38.96 & K \\
\hline
\end{tabular}




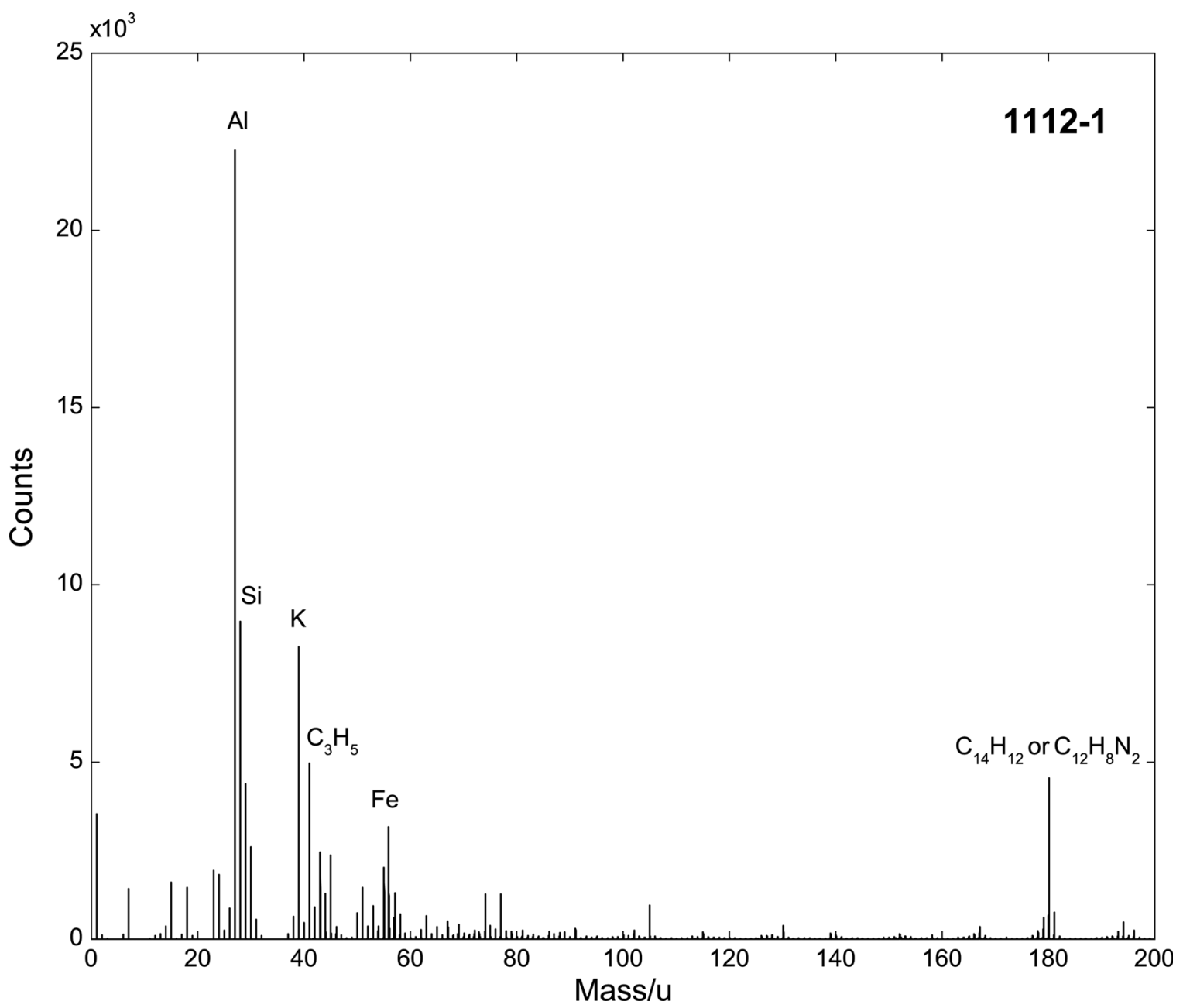

\begin{tabular}{rc}
\hline Accession \# & 01112-01 \\
\hline Host Material & Coal SS01 \\
Technique & ToF-SIMS \\
Mass Range & $200 \mathrm{Da}$ \\
Instrument & ION-TOF TOF-SIMS IV \\
Analyzer Type & time-of-flight, reflectron \\
Detector Type & $\sim 5000$ \\
Analyzer Mass Resolution & Electron multiplier \\
Specimen Normal to Analyzer & $0^{\circ}$ \\
Primary Beam lon Gun & ION-TOF \\
Primary Species & Ga \\
Primary lon Pulse Length & $25 \times 10^{-9}$ sec \\
Primary lon Pulse Rate & $10 \mathrm{kHz}$ \\
Net Beam Voltage & 25000 \\
Beam Current & $1.5 \mathrm{nA}$ \\
Beam Diameter & $0.1 \mu \mathrm{m}$ \\
Beam Raster Width & $500 \mu \mathrm{m} \times 500 \mu \mathrm{m}$ \\
Beam Incident Angle & $45^{\circ}\left(45^{\circ}\right.$ effective $)$ \\
Source to Analyzer Angle & $45^{\circ}$ \\
Comment & \\
\hline
\end{tabular}




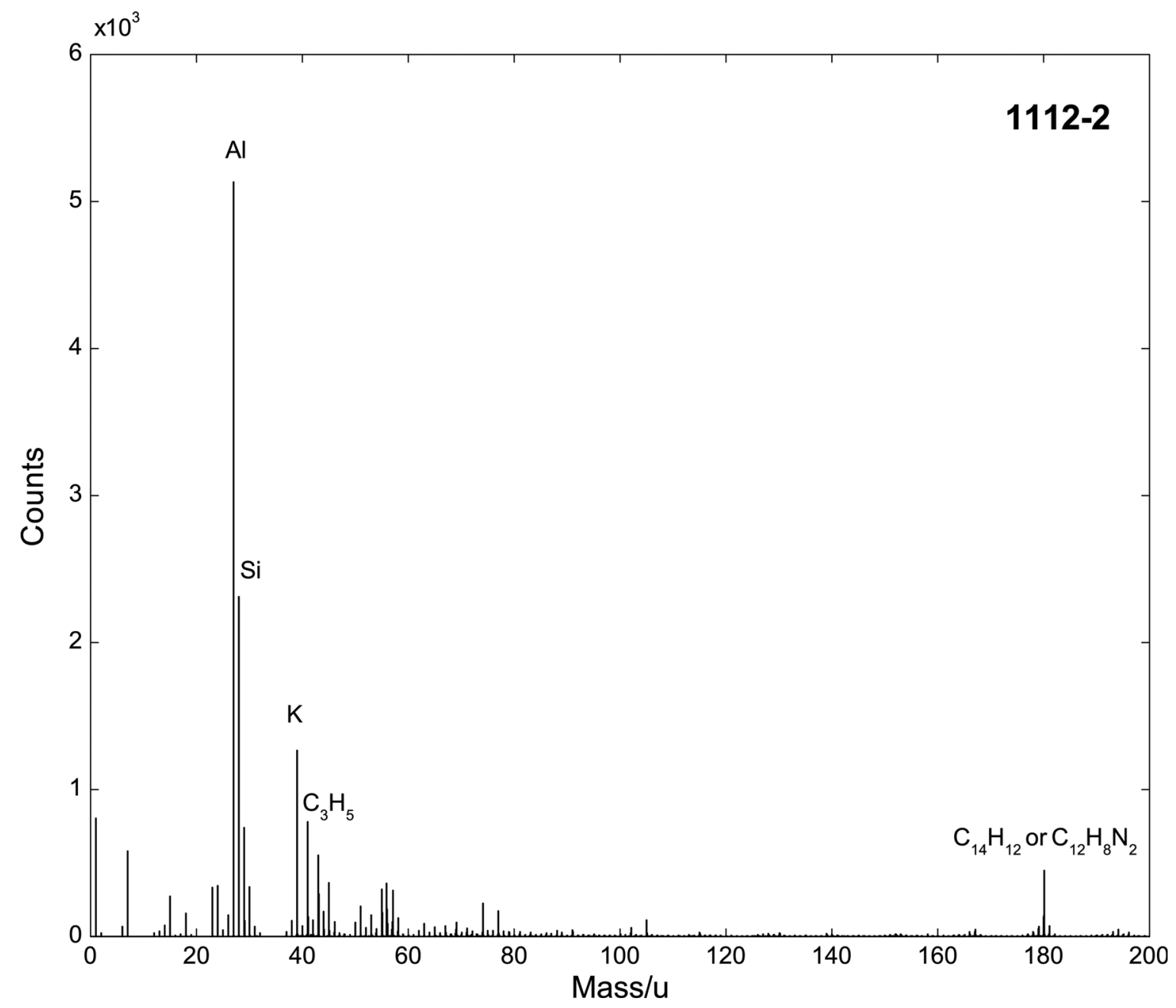

\begin{tabular}{rc}
\hline Accession \# & $\mathbf{0 1 1 1 2 - 0 2}$ \\
\hline Host Material & Coal SS01 \\
Technique & ToF-SIMS \\
Mass Range & $200 \mathrm{Da}$ \\
Instrument & ION-TOF TOF-SIMS IV \\
Analyzer Type & time-of-flight, reflectron \\
Detector Type & $\sim 5000$ \\
Analyzer Mass Resolution & Electron multiplier \\
Specimen Normal to Analyzer & $0^{\circ}$ \\
Primary Beam lon Gun & ION-TOF \\
Primary Species & Ga \\
Primary lon Pulse Length & $25 \times 10^{-9} \mathrm{sec}$ \\
Primary lon Pulse Rate & $10 \mathrm{kHz}$ \\
Net Beam Voltage & 25000 \\
Beam Current & $1.5 \mathrm{nA}$ \\
Beam Diameter & $0.1 \mu \mathrm{m}$ \\
Beam Raster Width & $500 \mu \mathrm{m} \times 500 \mu \mathrm{m}$ \\
Beam Incident Angle & $45^{\circ}\left(45^{\circ}\right.$ effective $)$ \\
Source to Analyzer Angle & $45^{\circ}$ \\
Comment & \\
\hline
\end{tabular}




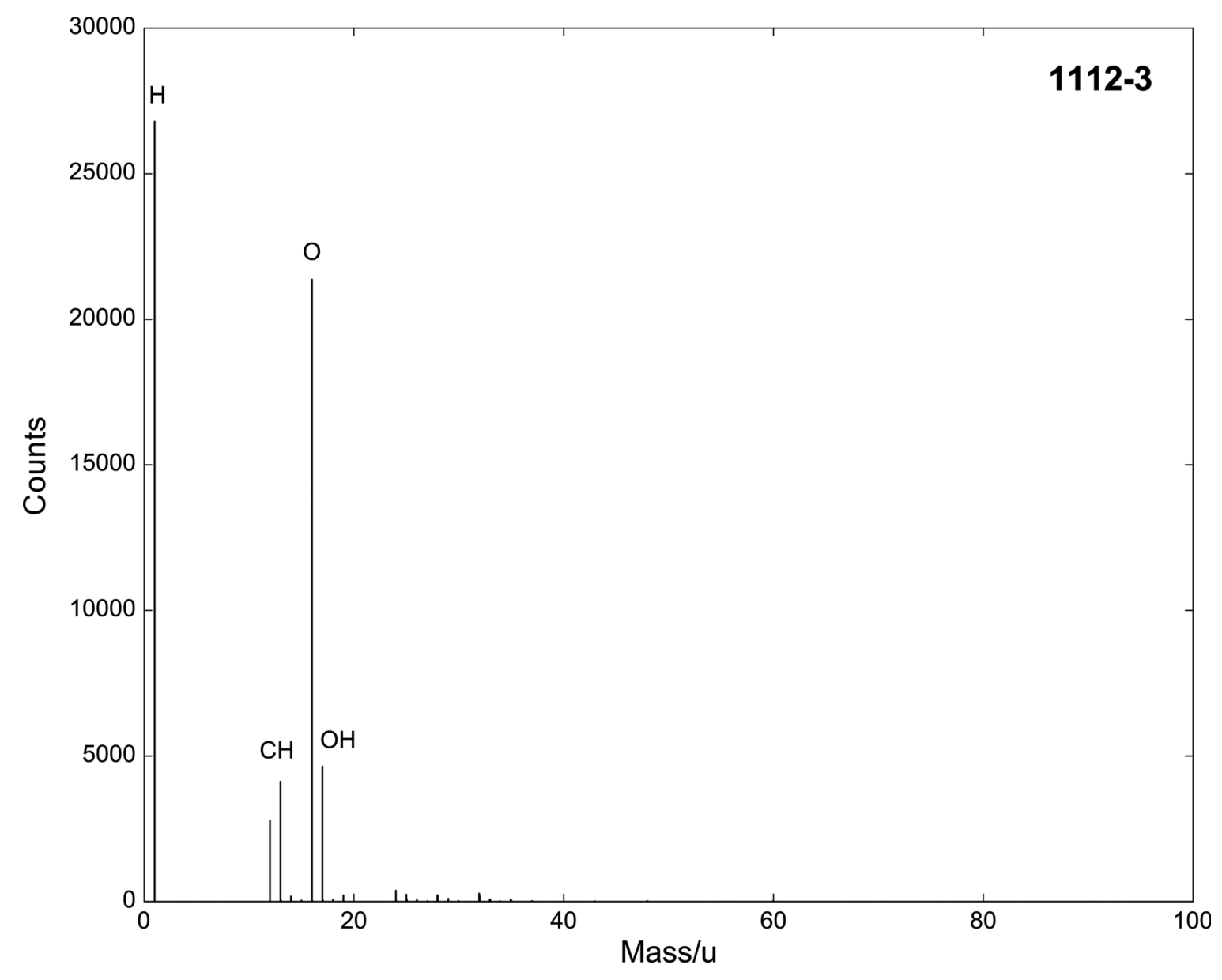

\begin{tabular}{rc}
\hline Accession \# & $\mathbf{0 1 1 1 2 - 0 3}$ \\
\hline Host Material & Coal SS01 \\
Technique & ToF-SIMS \\
Mass Range & $100 \mathrm{Da}$ \\
Instrument & ION-TOF TOF-SIMS IV \\
Analyzer Type & time-of-flight, reflectron \\
Detector Type & $\sim 5000$ \\
Analyzer Mass Resolution & Electron multiplier \\
Specimen Normal to Analyzer & $0^{\circ}$ \\
Primary Beam lon Gun & ION-TOF \\
Primary Species & $\mathrm{Ga}^{+}$ \\
Primary lon Pulse Length & $25 \times 10^{-9} \mathrm{sec}$ \\
Primary lon Pulse Rate & $10 \mathrm{kHz}$ \\
Net Beam Voltage & 25000 \\
Beam Current & $1.5 \mathrm{nA}$ \\
Beam Diameter & $0.1 \mu \mathrm{m}$ \\
Beam Raster Width & $500 \mu \mathrm{m} \times 500 \mu \mathrm{m}$ \\
Beam Incident Angle & $45^{\circ}\left(45^{\circ}\right.$ effective $)$ \\
Source to Analyzer Angle & $45^{\circ}$ \\
Comment & \\
\hline
\end{tabular}




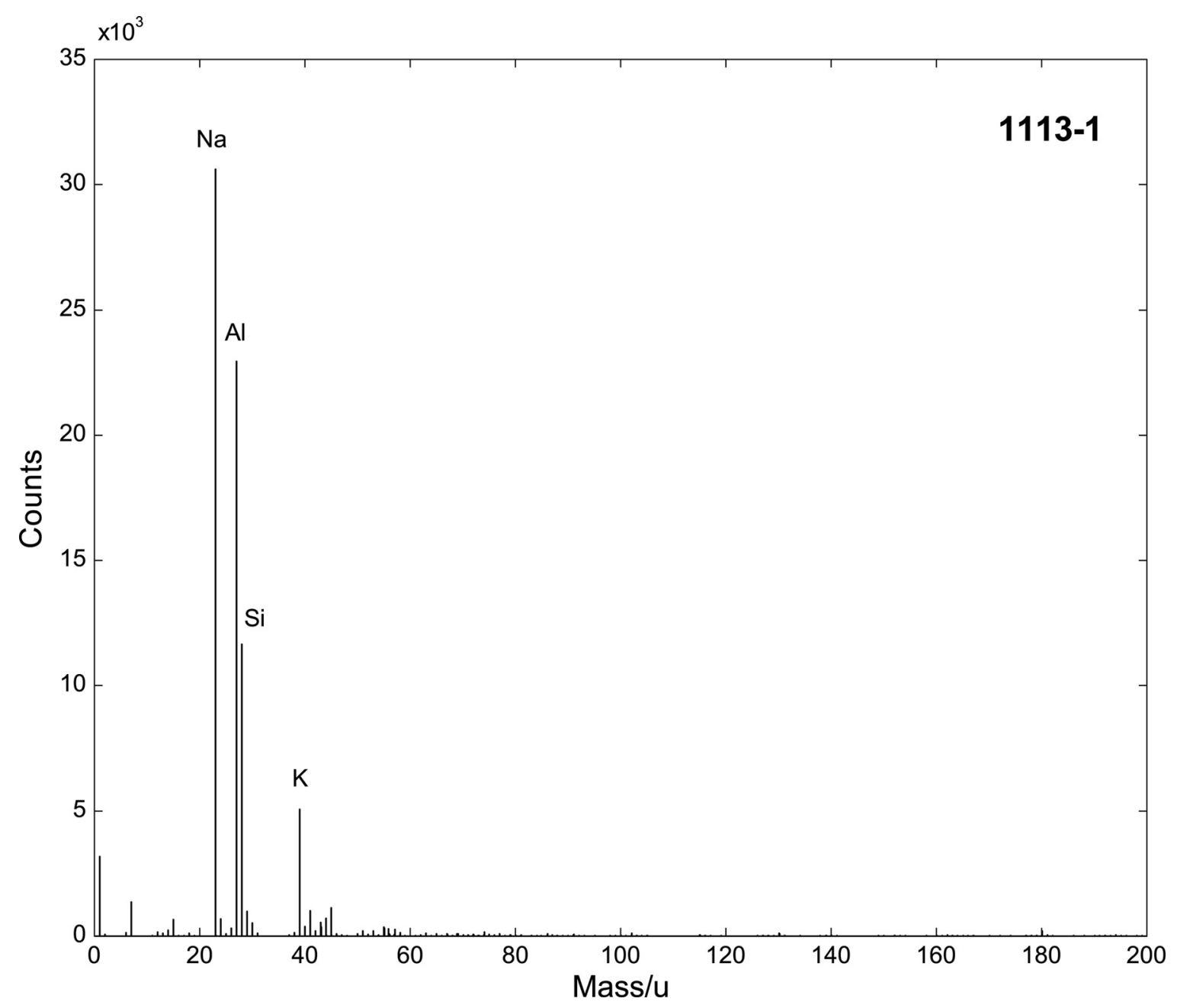

\begin{tabular}{rc}
\hline Accession \# & $\mathbf{0 1 1 1 3 - 0 1}$ \\
\hline Host Material & Coal SS02 \\
Technique & ToF-SIMS \\
Mass Range & $200 \mathrm{Da}$ \\
Instrument & ION-TOF TOF-SIMS IV \\
Analyzer Type & time-of-flight, reflectron \\
Detector Type & $\sim 5000$ \\
Analyzer Mass Resolution & Electron multiplier \\
Specimen Normal to Analyzer & $0^{\circ}$ \\
Primary Beam lon Gun & ON-TOF \\
Primary Species & $\mathrm{Ga}^{+}$ \\
Primary lon Pulse Length & $25 \times 10^{-9} \mathrm{sec}$ \\
Primary lon Pulse Rate & $10 \mathrm{kHz}$ \\
Net Beam Voltage & 25000 \\
Beam Current & $1.5 \mathrm{nA}$ \\
Beam Diameter & $0.1 \mu \mathrm{m}$ \\
Beam Raster Width & $500 \mu \mathrm{m} \times 500 \mu \mathrm{m}$ \\
Beam Incident Angle & $45^{\circ}\left(45^{\circ}\right.$ effective $)$ \\
Source to Analyzer Angle & $45^{\circ}$ \\
Comment & \\
\hline
\end{tabular}




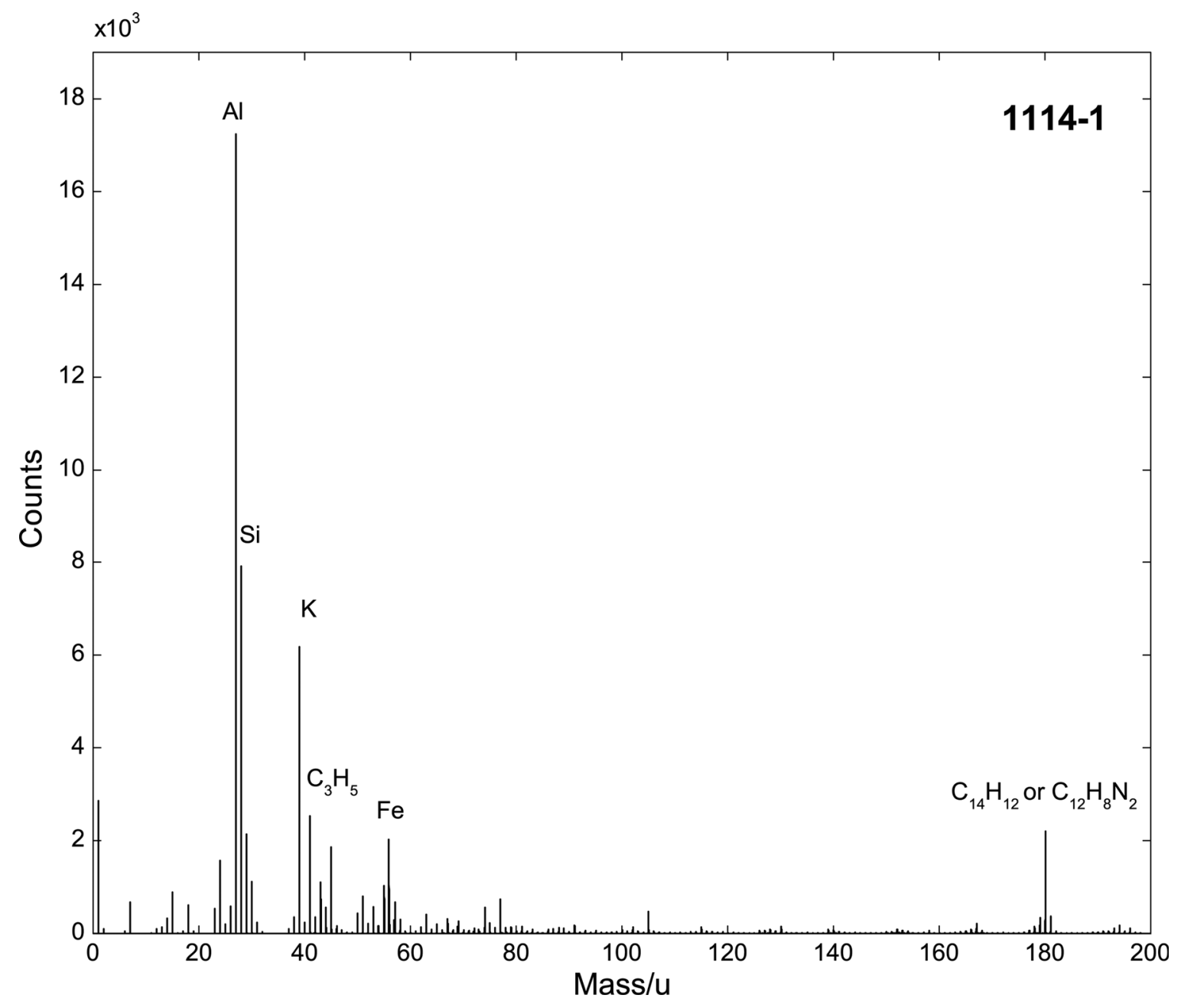

\begin{tabular}{rc}
\hline Accession \# & $\mathbf{0 1 1 1 4 - 0 1}$ \\
\hline Host Material & Coal SS03 \\
Technique & ToF-SIMS \\
Mass Range & 200 Da \\
Instrument & ION-TOF TOF-SIMS IV \\
Analyzer Type & time-of-flight, reflectron \\
Detector Type & $\sim 5000$ \\
Analyzer Mass Resolution & Electron multiplier \\
Specimen Normal to Analyzer & $0^{\circ}$ \\
Primary Beam lon Gun & ION-TOF \\
Primary Species & Ga \\
Primary lon Pulse Length & $25 \times 10^{-9}$ sec \\
Primary lon Pulse Rate & $10 \mathrm{kHz}$ \\
Net Beam Voltage & 25000 \\
Beam Current & $1.5 \mathrm{nA}$ \\
Beam Diameter & $0.1 \mu \mathrm{m}$ \\
Beam Raster Width & $500 \mu \mathrm{m} \times 500 \mu \mathrm{m}$ \\
Beam Incident Angle & $45^{\circ}\left(45^{\circ}\right.$ effective $)$ \\
Source to Analyzer Angle & $45^{\circ}$ \\
Comment & \\
\hline
\end{tabular}




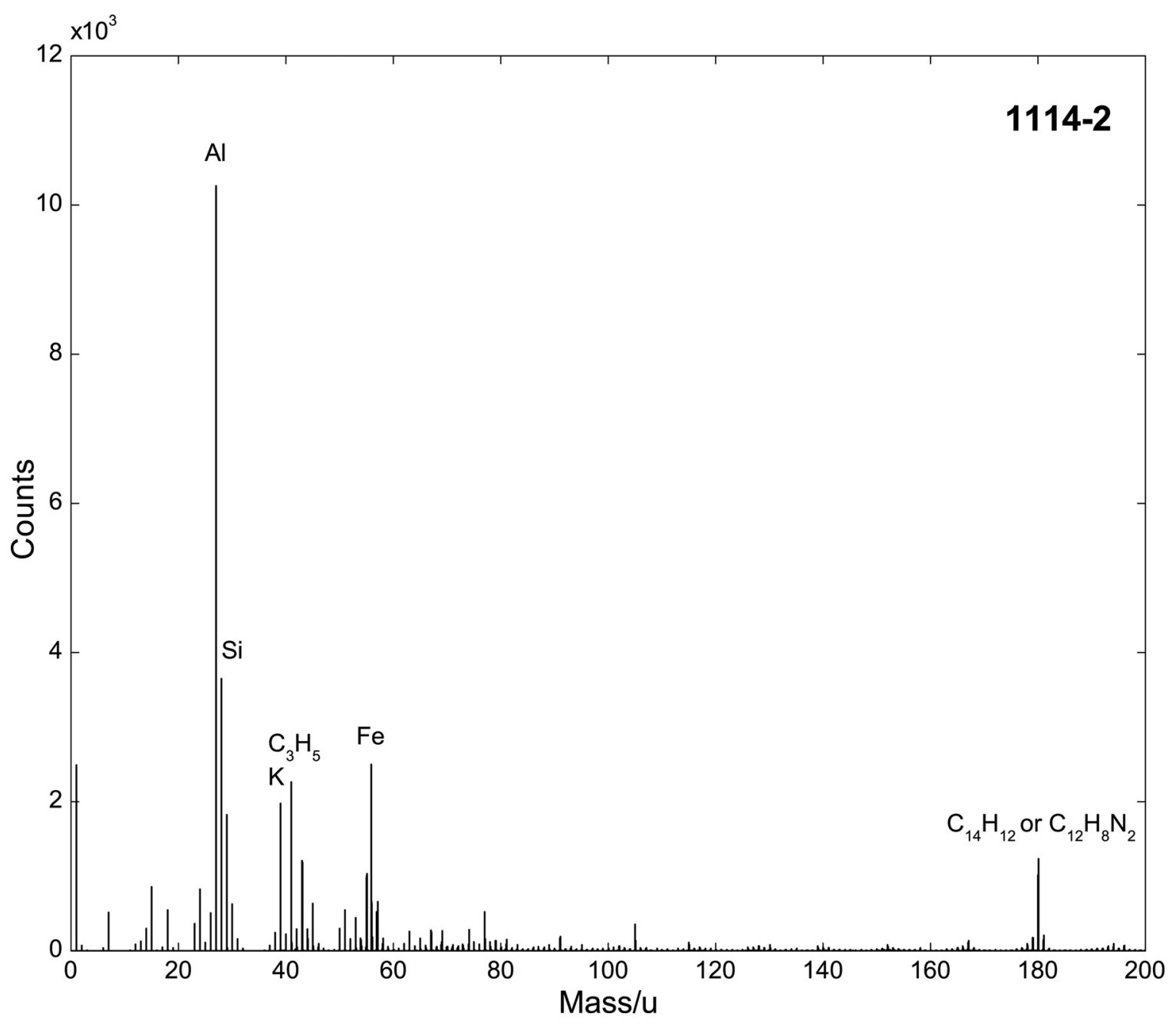

\begin{tabular}{rc}
\hline Accession \# & 01114-02 \\
\hline Host Material & Coal SS03 \\
Technique & ToF-SIMS \\
Mass Range & $200 \mathrm{Da}$ \\
Instrument & ION-TOF TOF-SIMS IV \\
Analyzer Type & time-of-flight, reflectron \\
Detector Type & $\sim 5000$ \\
Analyzer Mass Resolution & Electron multiplier \\
Specimen Normal to Analyzer & $0^{\circ}$ \\
Primary Beam lon Gun & ION-TOF \\
Primary Species & Ga \\
Primary lon Pulse Length & $25 \times 10^{-9}$ sec \\
Primary lon Pulse Rate & $10 \mathrm{kHz}$ \\
Net Beam Voltage & 25000 \\
Beam Current & $1.5 \mathrm{nA}$ \\
Beam Diameter & $0.1 \mu \mathrm{m}$ \\
Beam Raster Width & $500 \mu \mathrm{m} \times 500 \mu \mathrm{m}$ \\
Beam Incident Angle & $45^{\circ}\left(45^{\circ}\right.$ effective $)$ \\
Source to Analyzer Angle & $45^{\circ}$ \\
Comment & \\
\hline
\end{tabular}




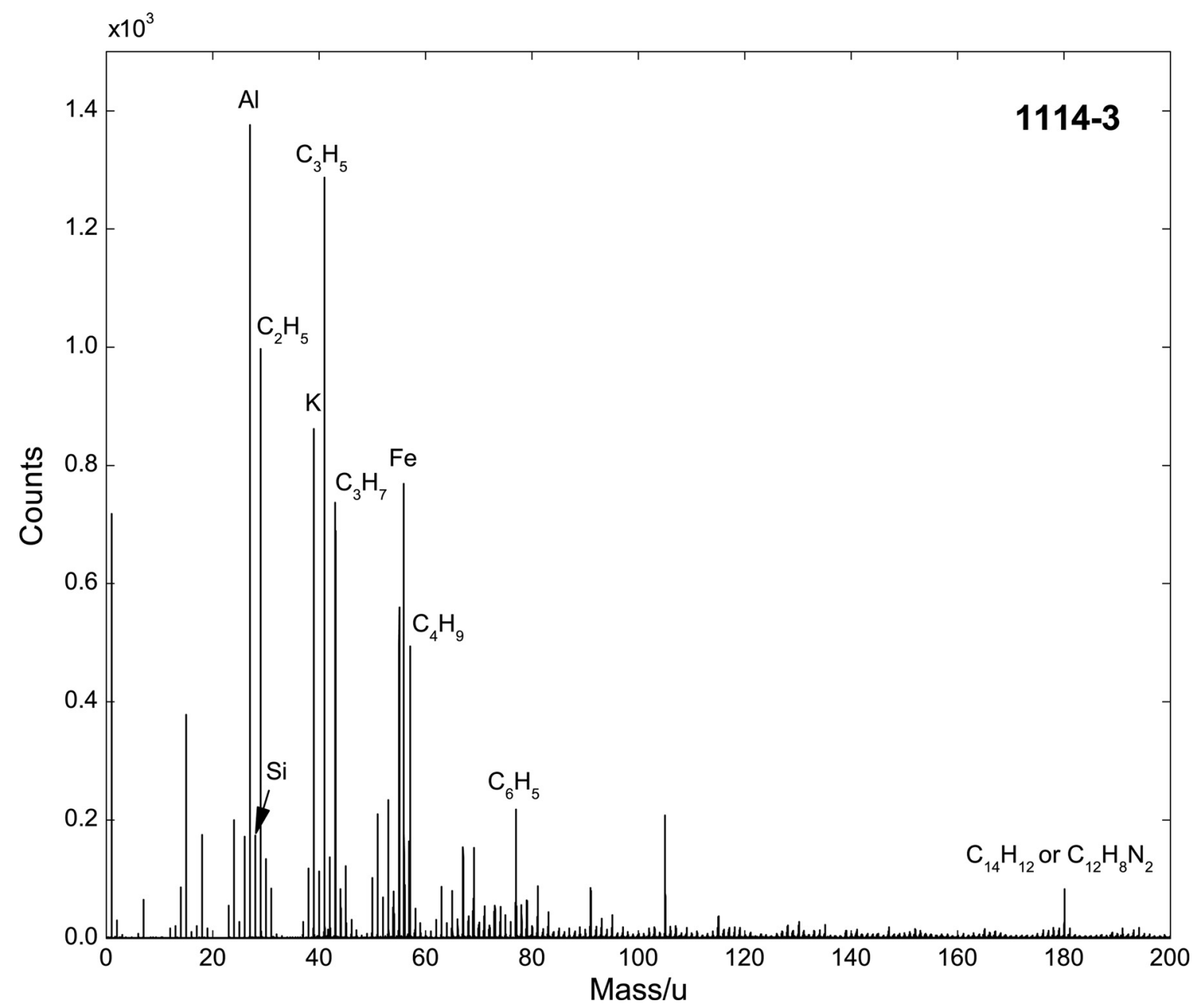

\begin{tabular}{rc}
\hline Accession \# & $\mathbf{0 1 1 1 4 - 0 3}$ \\
\hline Host Material & Coal SS03 \\
Technique & ToF-SIMS \\
Mass Range & $200 \mathrm{Da}$ \\
Instrument & ION-TOF TOF-SIMS IV \\
Analyzer Type & time-of-flight, reflectron \\
Detector Type & $\sim 5000$ \\
Analyzer Mass Resolution & Electron multiplier \\
Specimen Normal to Analyzer & $0^{\circ}$ \\
Primary Beam lon Gun & ION-TOF \\
Primary Species & Ga ${ }^{+}$ \\
Primary lon Pulse Length & $25 \times 10^{-9}$ sec \\
Primary lon Pulse Rate & $10 \mathrm{kHz}$ \\
Net Beam Voltage & 25000 \\
Beam Current & $1.5 \mathrm{nA}$ \\
Beam Diameter & $0.1 \mu \mathrm{m}$ \\
Beam Raster Width & $500 \mu \mathrm{m} \times 500 \mu \mathrm{m}$ \\
Beam Incident Angle & $45^{\circ}\left(45^{\circ}\right.$ effective $)$ \\
Source to Analyzer Angle & $45^{\circ}$ \\
Comment & \\
\hline
\end{tabular}




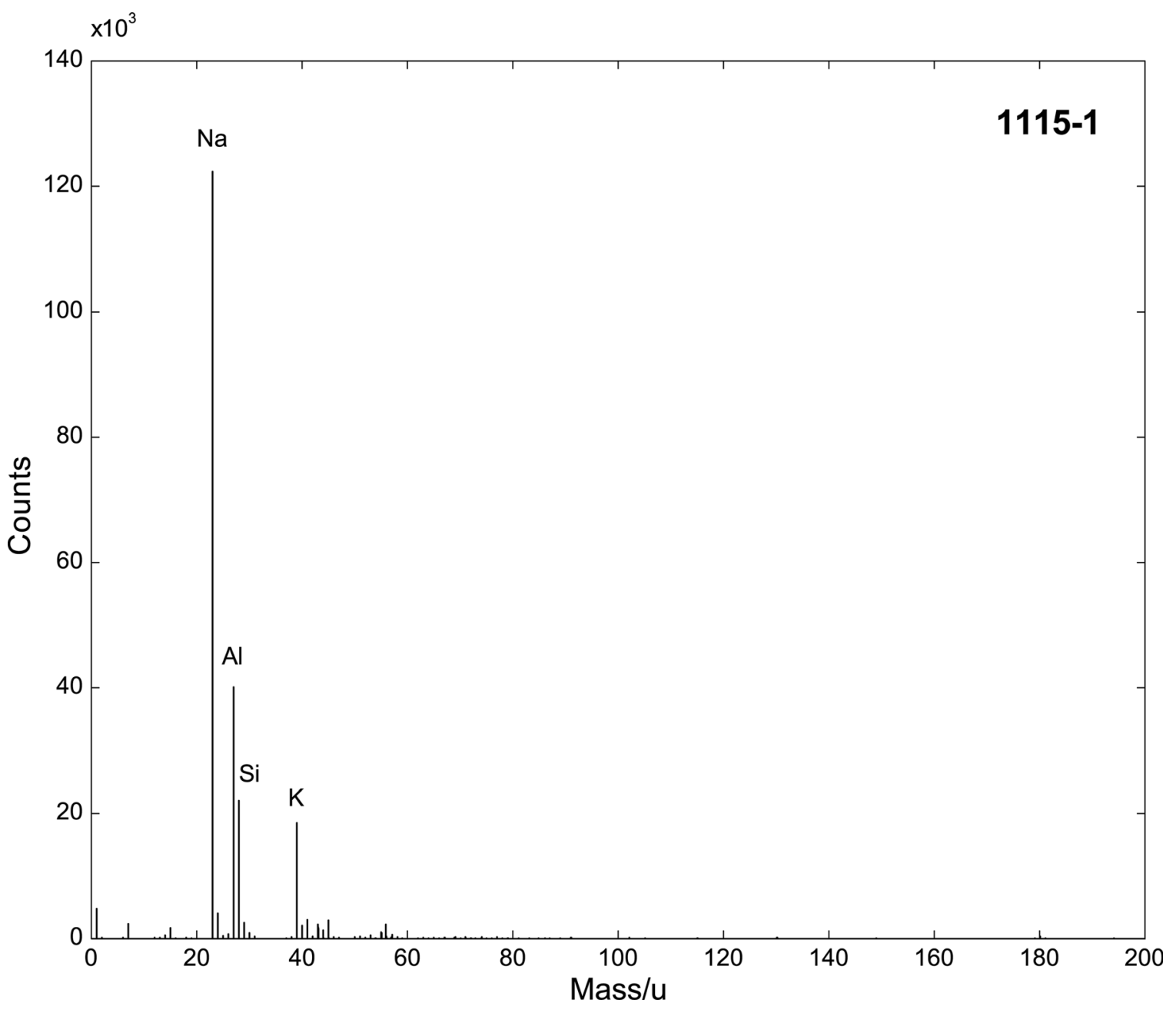

\begin{tabular}{rc}
\hline Accession \# & $\mathbf{0 1 1 1 5 - 0 1}$ \\
\hline Host Material & Coal SS04 \\
Technique & ToF-SIMS \\
Mass Range & $200 \mathrm{Da}$ \\
Instrument & ION-TOF TOF-SIMS IV \\
Analyzer Type & time-of-flight, reflectron \\
Detector Type & $\sim 5000$ \\
Analyzer Mass Resolution & Electron multiplier \\
Specimen Normal to Analyzer & $0^{\circ}$ \\
Primary Beam lon Gun & ON-TOF \\
Primary Species & Ga \\
Primary lon Pulse Length & $25 \times 10^{-9} \mathrm{sec}$ \\
Primary lon Pulse Rate & $10 \mathrm{kHz}$ \\
Net Beam Voltage & 25000 \\
Beam Current & $1.5 \mathrm{nA}$ \\
Beam Diameter & $0.1 \mu \mathrm{m}$ \\
Beam Raster Width & $500 \mu \mathrm{m} \times 500 \mu \mathrm{m}$ \\
Beam Incident Angle & $45^{\circ}\left(45^{\circ}\right.$ effective $)$ \\
Source to Analyzer Angle & $45^{\circ}$ \\
Comment & \\
\hline
\end{tabular}




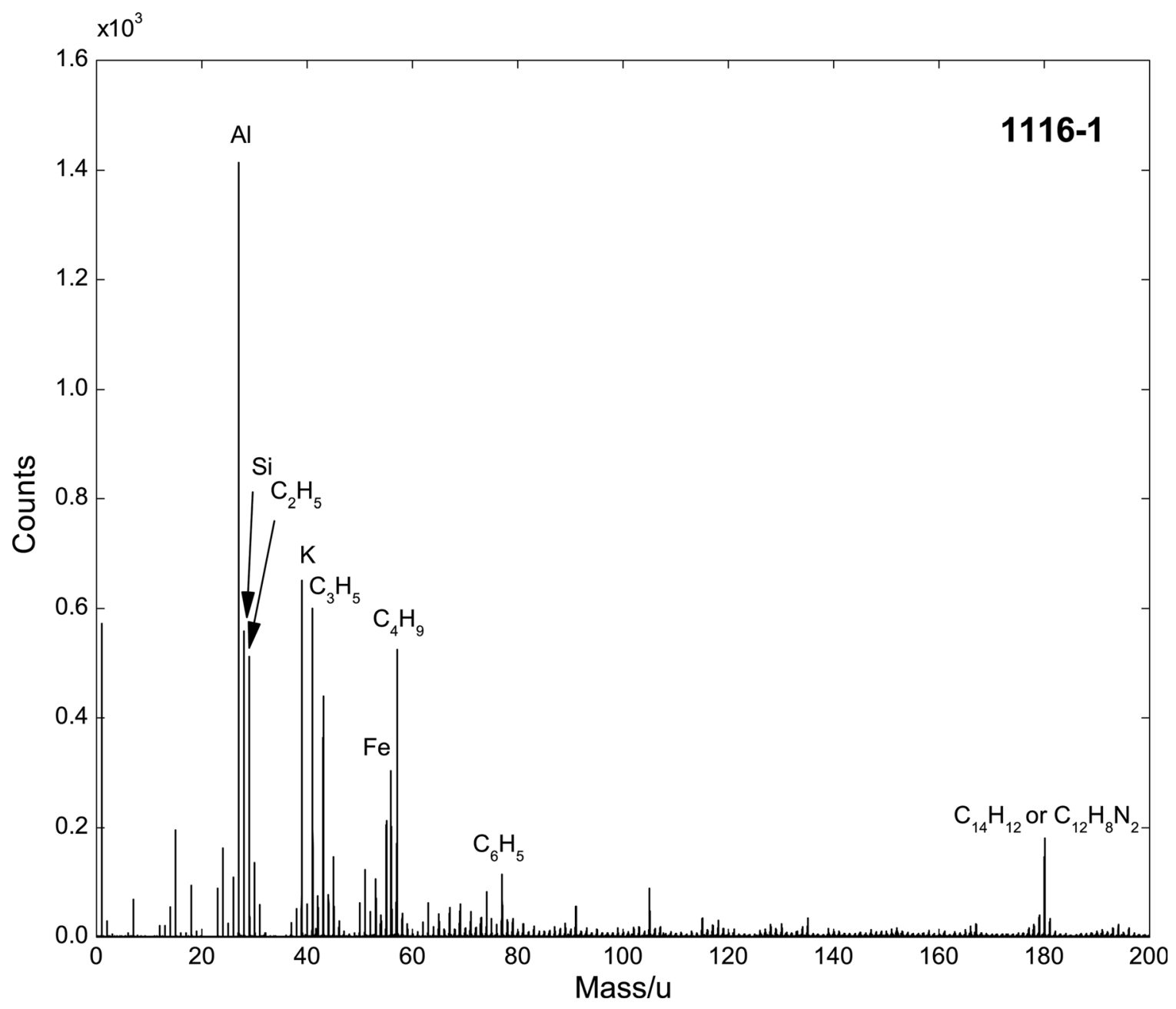

\begin{tabular}{rc}
\hline Accession \# & 01116-01 \\
\hline Host Material & Coal SS05 \\
Technique & ToF-SIMS \\
Mass Range & 200 Da \\
Instrument & ION-TOF TOF-SIMS IV \\
Analyzer Type & time-of-flight, reflectron \\
Detector Type & $\sim 5000$ \\
Analyzer Mass Resolution & Electron multiplier \\
Specimen Normal to Analyzer & $0^{\circ}$ \\
Primary Beam lon Gun & OON-TOF \\
Primary Species & Ga \\
Primary lon Pulse Length & $25 \times 10^{-9} \mathrm{sec}$ \\
Primary lon Pulse Rate & $10 \mathrm{kHz}$ \\
Net Beam Voltage & 25000 \\
Beam Current & $1.5 \mathrm{nA}$ \\
Beam Diameter & $0.1 \mu \mathrm{m}$ \\
Beam Raster Width & $500 \mu \mathrm{m} \times 500 \mu \mathrm{m}$ \\
Beam Incident Angle & $45^{\circ}\left(45^{\circ}\right.$ effective $)$ \\
Source to Analyzer Angle & $45^{\circ}$ \\
Comment & \\
\hline
\end{tabular}




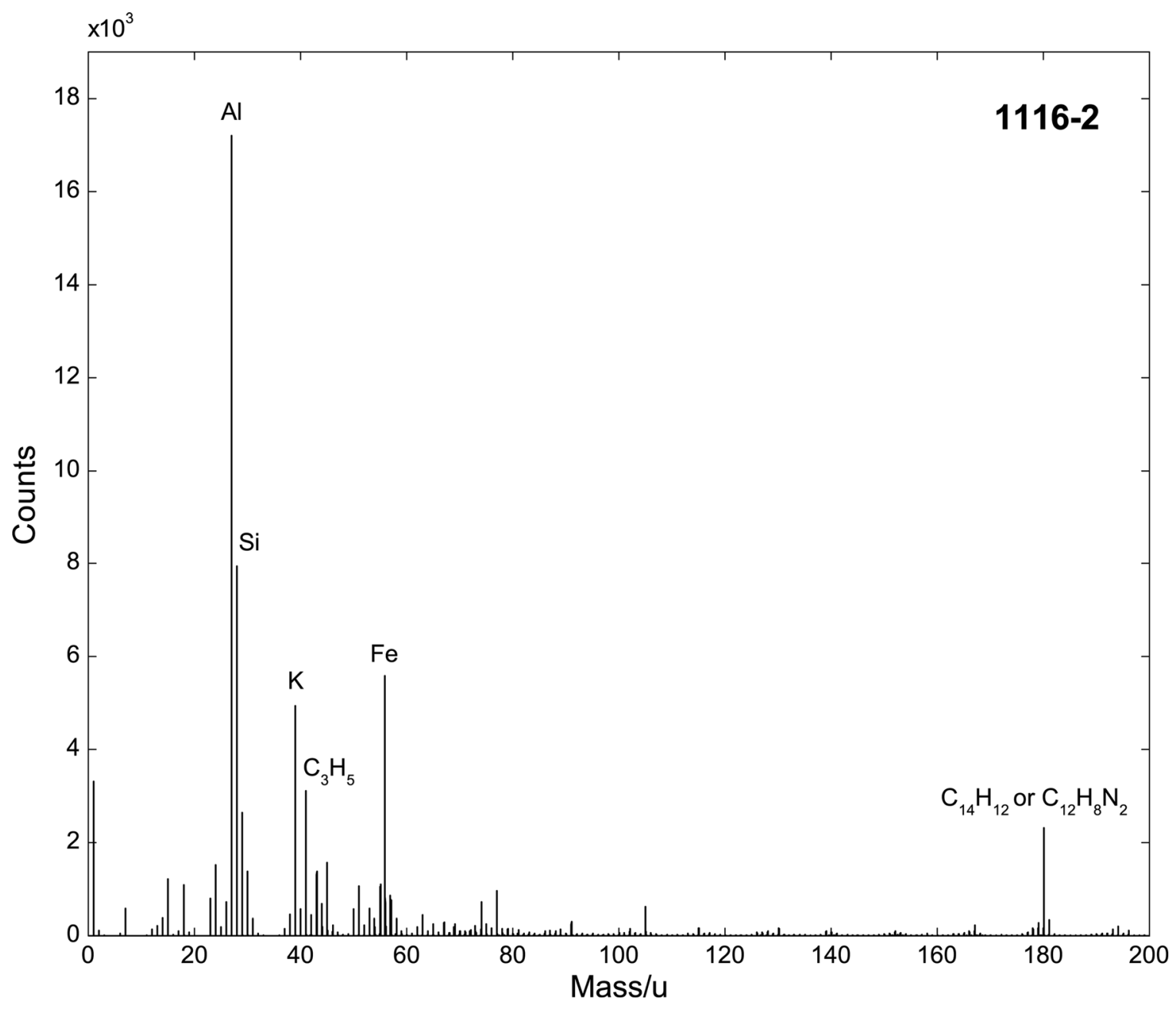

\begin{tabular}{rc}
\hline Accession \# & 01116-02 \\
\hline Host Material & Coal SS05 \\
Technique & ToF-SIMS \\
Mass Range & $200 \mathrm{Da}$ \\
Instrument & ION-TOF TOF-SIMS IV \\
Analyzer Type & time-of-flight, reflectron \\
Detector Type & $\sim 5000$ \\
Analyzer Mass Resolution & Electron multiplier \\
Specimen Normal to Analyzer & $0^{\circ}$ \\
Primary Beam lon Gun & ION-TOF \\
Primary Species & Ga \\
Primary lon Pulse Length & $25 \times 10^{-9}$ sec \\
Primary lon Pulse Rate & $10 \mathrm{kHz}$ \\
Net Beam Voltage & 25000 \\
Beam Current & $1.5 \mathrm{nA}$ \\
Beam Diameter & $0.1 \mu \mathrm{m}$ \\
Beam Raster Width & $500 \mu \mathrm{m} \times 500 \mu \mathrm{m}$ \\
Beam Incident Angle & $45^{\circ}\left(45^{\circ}\right.$ effective $)$ \\
Source to Analyzer Angle & $45^{\circ}$ \\
Comment & \\
\hline
\end{tabular}




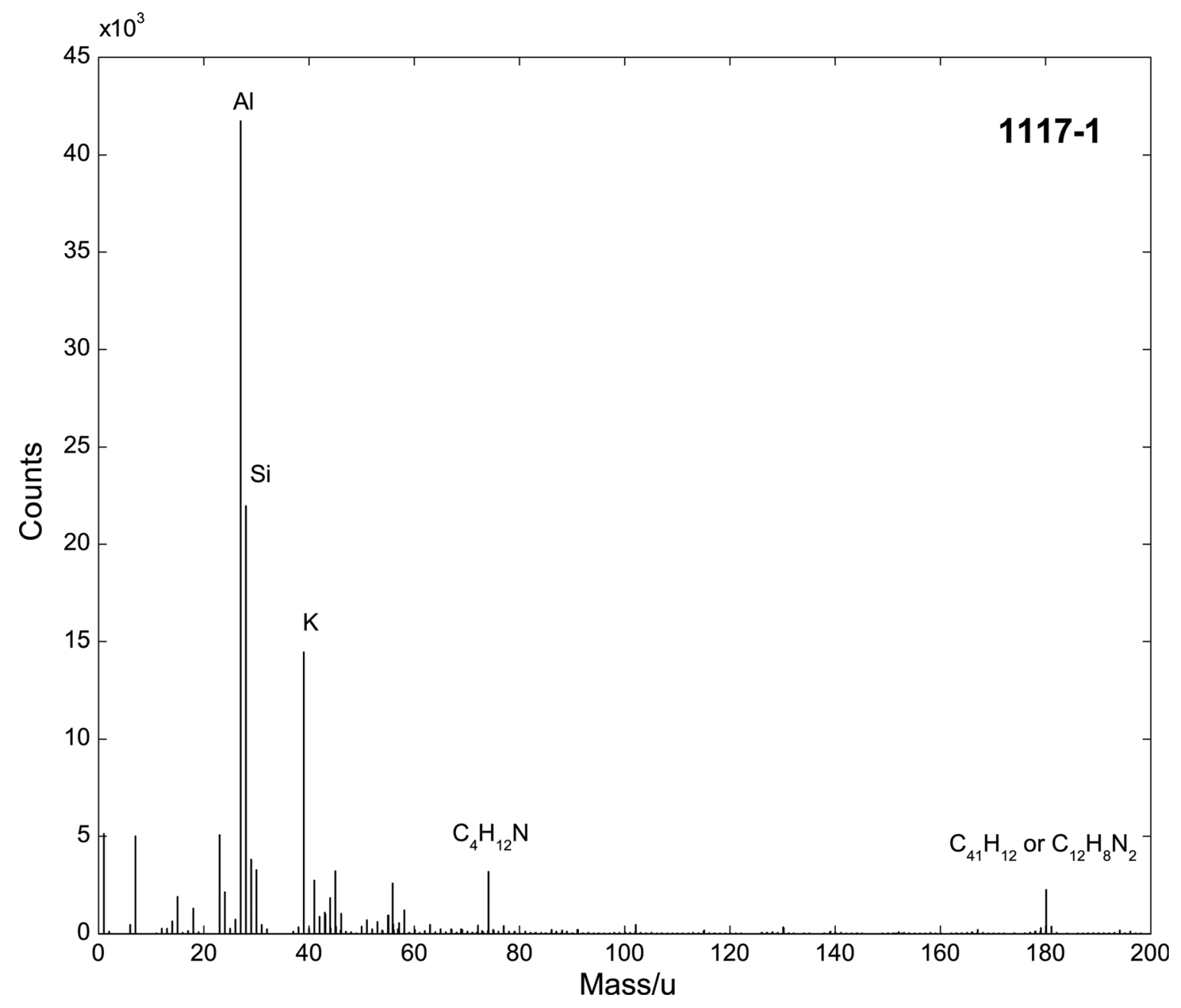

\begin{tabular}{rc}
\hline Accession \# & $\mathbf{0 1 1 1 7 - 0 1}$ \\
\hline Host Material & Coal SS06 \\
Technique & ToF-SIMS \\
Mass Range & 200 Da \\
Instrument & ION-TOF TOF-SIMS IV \\
Analyzer Type & time-of-flight, reflectron \\
Detector Type & $\sim 5000$ \\
Analyzer Mass Resolution & Electron multiplier \\
Specimen Normal to Analyzer & $0^{\circ}$ \\
Primary Beam lon Gun & ION-TOF \\
Primary Species & Ga \\
Primary lon Pulse Length & $25 \times 10^{-9}$ sec \\
Primary lon Pulse Rate & $10 \mathrm{kHz}$ \\
Net Beam Voltage & 25000 \\
Beam Current & $1.5 \mathrm{nA}$ \\
Beam Diameter & $0.1 \mu \mathrm{m}$ \\
Beam Raster Width & $500 \mu \mathrm{m} \times 500 \mu \mathrm{m}$ \\
Beam Incident Angle & $45^{\circ}\left(45^{\circ}\right.$ effective $)$ \\
Source to Analyzer Angle & $45^{\circ}$ \\
Comment & \\
\hline
\end{tabular}




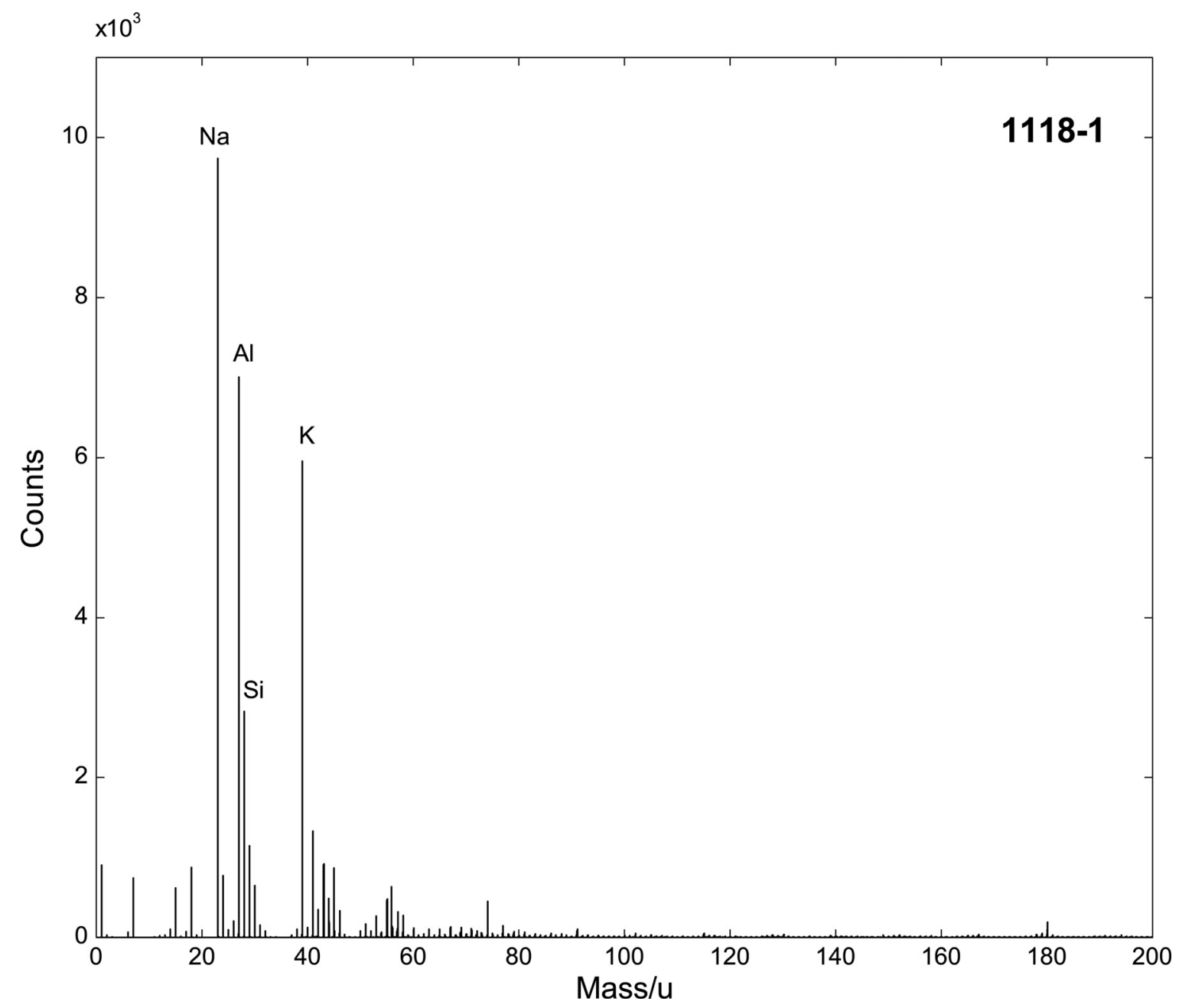

\begin{tabular}{|c|c|}
\hline Accession \# & 01118-01 \\
\hline Host Material & Coal SS07 \\
\hline Technique & ToF-SIMS \\
\hline Mass Range & $200 \mathrm{Da}$ \\
\hline Instrument & ION-TOF TOF-SIMS IV \\
\hline Analyzer Type & time-of-flight, reflectron \\
\hline Analyzer Mass Resolution & $\sim 5000$ \\
\hline Detector Type & Electron multiplier \\
\hline Specimen Normal to Analyzer & $0^{\circ}$ \\
\hline Primary Beam Ion Gun & ION-TOF \\
\hline Primary Species & $\mathrm{Ga}^{+}$ \\
\hline Primary Ion Pulse Length & $25 \times 10^{-9} \mathrm{sec}$ \\
\hline Primary Ion Pulse Rate & $10 \mathrm{kHz}$ \\
\hline Net Beam Voltage & 25000 \\
\hline Beam Current & $1.5 \mathrm{nA}$ \\
\hline Beam Diameter & $0.1 \mu \mathrm{m}$ \\
\hline Beam Raster Width & $500 \mu \mathrm{m} \times 500 \mu \mathrm{m}$ \\
\hline Beam Incident Angle & $45^{\circ}\left(45^{\circ}\right.$ effective $)$ \\
\hline Source to Analyzer Angle & $45^{\circ}$ \\
\hline Comment & \\
\hline
\end{tabular}




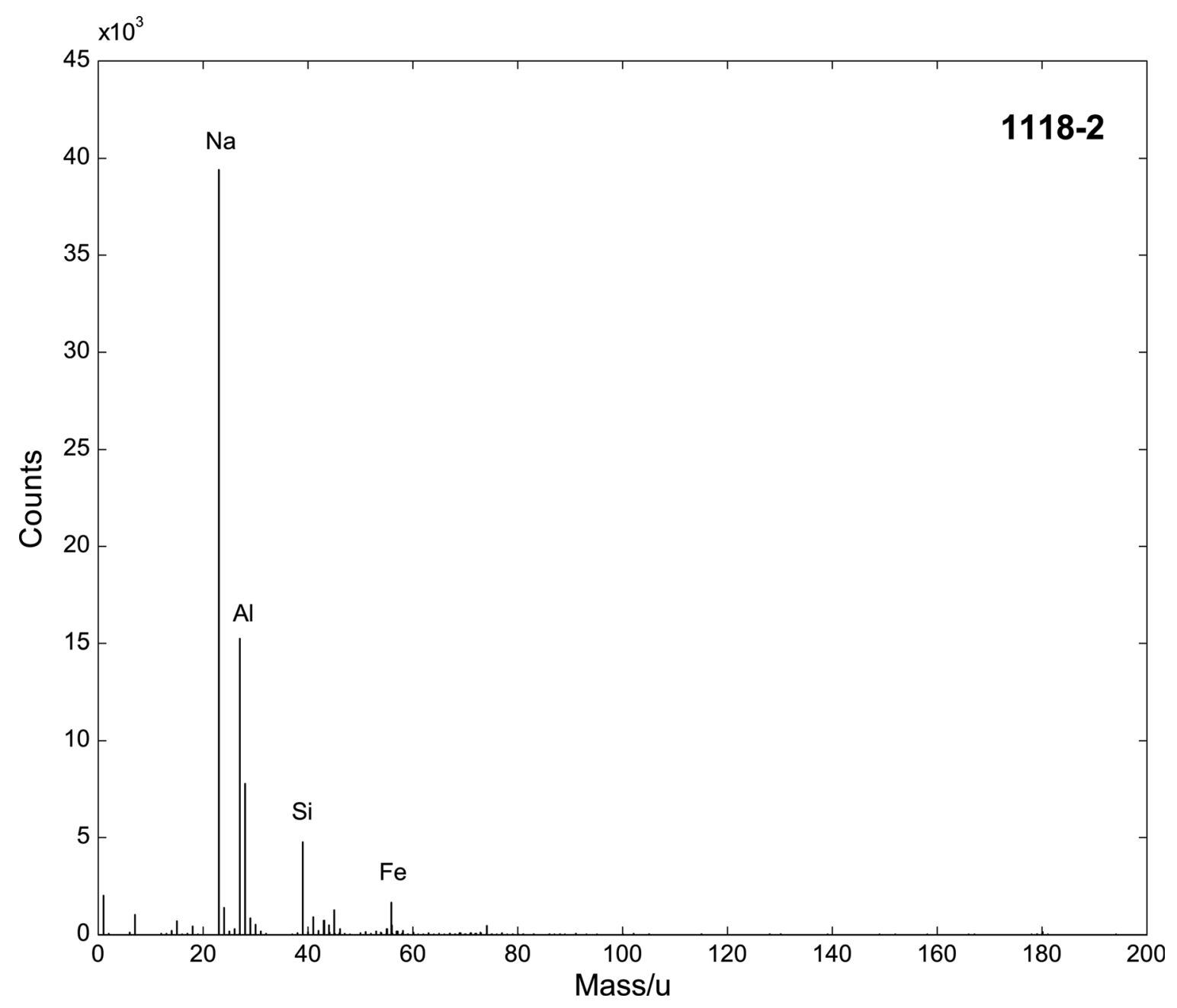

\begin{tabular}{rc}
\hline Accession \# & $\mathbf{0 1 1 1 8 - 0 2}$ \\
\hline Host Material & Coal SSO7 \\
Technique & ToF-SIMS \\
Mass Range & $200 \mathrm{Da}$ \\
Instrument & ION-TOF TOF-SIMS IV \\
Analyzer Type & time-of-flight, reflectron \\
Detector Type & $\sim 5000$ \\
Analyzer Mass Resolution & Electron multiplier \\
Specimen Normal to Analyzer & $0^{\circ}$ \\
Primary Beam lon Gun & ION-TOF \\
Primary Species & $\mathrm{Ga}^{+}$ \\
Primary lon Pulse Length & $25 \times 10^{-9} \mathrm{sec}$ \\
Primary lon Pulse Rate & $10 \mathrm{kHz}$ \\
Net Beam Voltage & 25000 \\
Beam Current & $1.5 \mathrm{nA}$ \\
Beam Diameter & $0.1 \mu \mathrm{m}$ \\
Beam Raster Width & $500 \mu \mathrm{m} \times 500 \mu \mathrm{m}$ \\
Beam Incident Angle & $45^{\circ}\left(45^{\circ}\right.$ effective $)$ \\
Source to Analyzer Angle & $45^{\circ}$ \\
Comment & \\
\hline
\end{tabular}




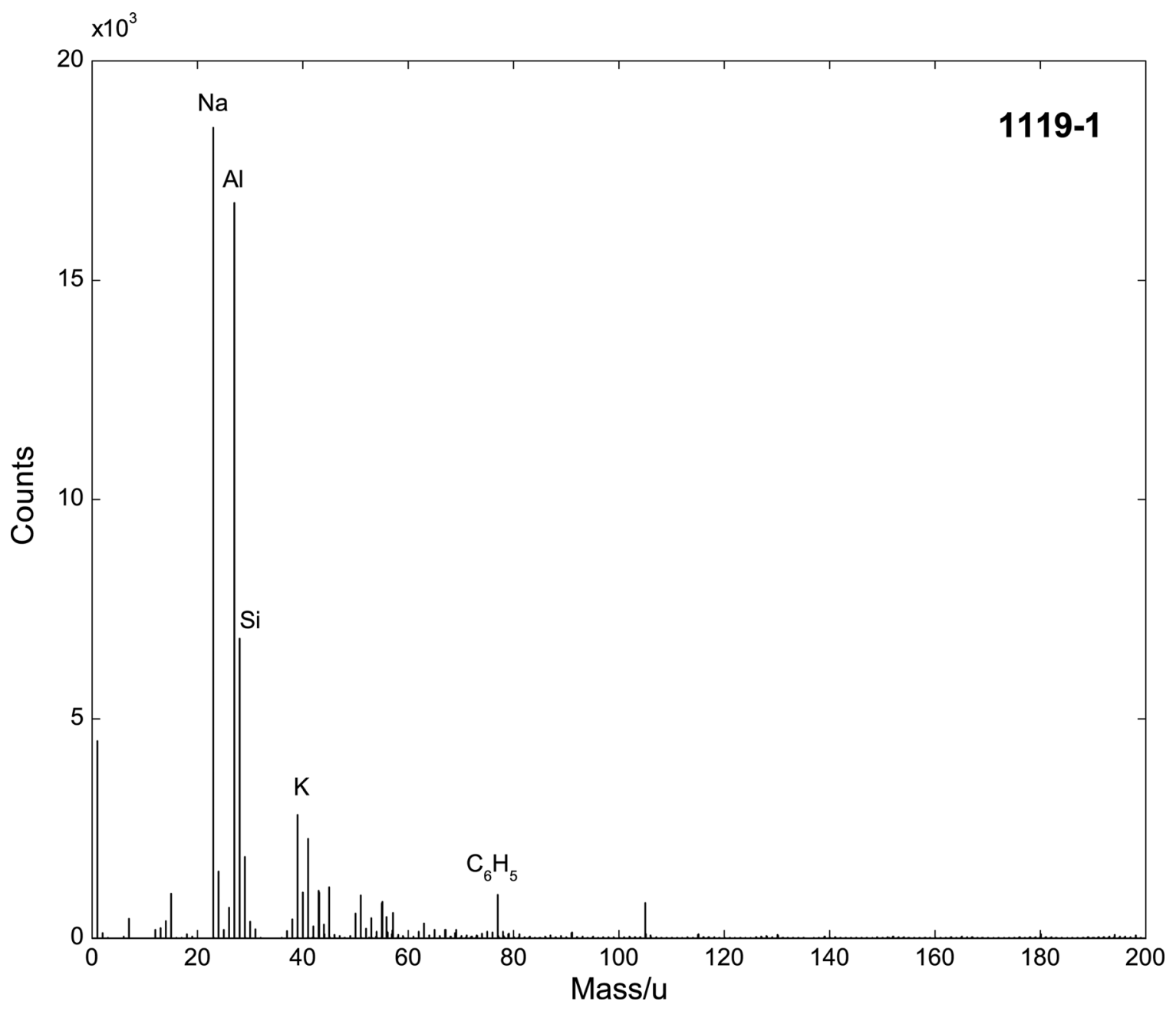

\begin{tabular}{rc}
\hline Accession \# & $\mathbf{0 1 1 1 9 - 0 1}$ \\
\hline Host Material & Coal SS08 \\
Technique & ToF-SIMS \\
Mass Range & 200 Da \\
Instrument & ION-TOF TOF-SIMS IV \\
Analyzer Type & time-of-flight, reflectron \\
Detector Type & $\sim 5000$ \\
Analyzer Mass Resolution & Electron multiplier \\
Specimen Normal to Analyzer & $0^{\circ}$ \\
Primary Beam lon Gun & ION-TOF \\
Primary Species & Ga \\
Primary lon Pulse Length & $25 \times 10^{-9} \mathrm{sec}$ \\
Primary lon Pulse Rate & $10 \mathrm{kHz}$ \\
Net Beam Voltage & 25000 \\
Beam Current & $1.5 \mathrm{nA}$ \\
Beam Diameter & $0.1 \mu \mathrm{m}$ \\
Beam Raster Width & $500 \mu \mathrm{m} \times 500 \mu \mathrm{m}$ \\
Beam Incident Angle & $45^{\circ}\left(45^{\circ}\right.$ effective $)$ \\
Source to Analyzer Angle & $45^{\circ}$ \\
Comment & \\
\hline
\end{tabular}




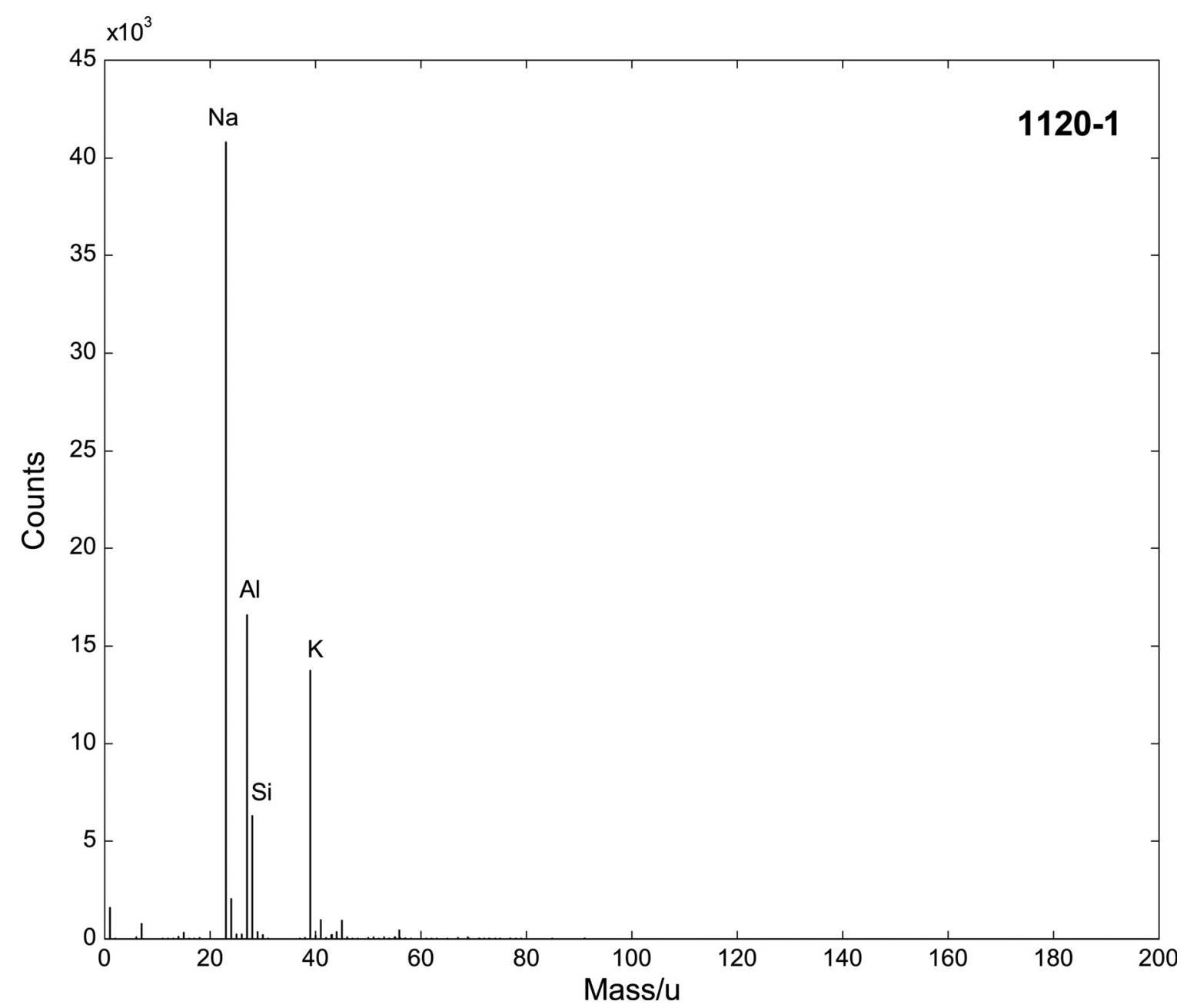

\begin{tabular}{rc}
\hline Accession \# & $\mathbf{0 1 1 2 0 - 0 1}$ \\
\hline Host Material & Coal SS09 \\
Technique & ToF-SIMS \\
Mass Range & $200 \mathrm{Da}$ \\
Instrument & ION-TOF TOF-SIMS IV \\
Analyzer Type & time-of-flight, reflectron \\
Detector Type & $\sim 5000$ \\
Analyzer Mass Resolution & Electron multiplier \\
Specimen Normal to Analyzer & $0^{\circ}$ \\
Primary Beam lon Gun & ION-TOF \\
Primary Species & $\mathrm{Ga}^{+}$ \\
Primary lon Pulse Length & $25 \times 10^{-9} \mathrm{sec}$ \\
Primary lon Pulse Rate & $10 \mathrm{kHz}$ \\
Net Beam Voltage & 25000 \\
Beam Current & $1.5 \mathrm{nA}$ \\
Beam Diameter & $0.1 \mu \mathrm{m}$ \\
Beam Raster Width & $500 \mu \mathrm{m} \times 500 \mu \mathrm{m}$ \\
Beam Incident Angle & $45^{\circ}\left(45^{\circ}\right.$ effective $)$ \\
Source to Analyzer Angle & $45^{\circ}$ \\
Comment & \\
\hline
\end{tabular}




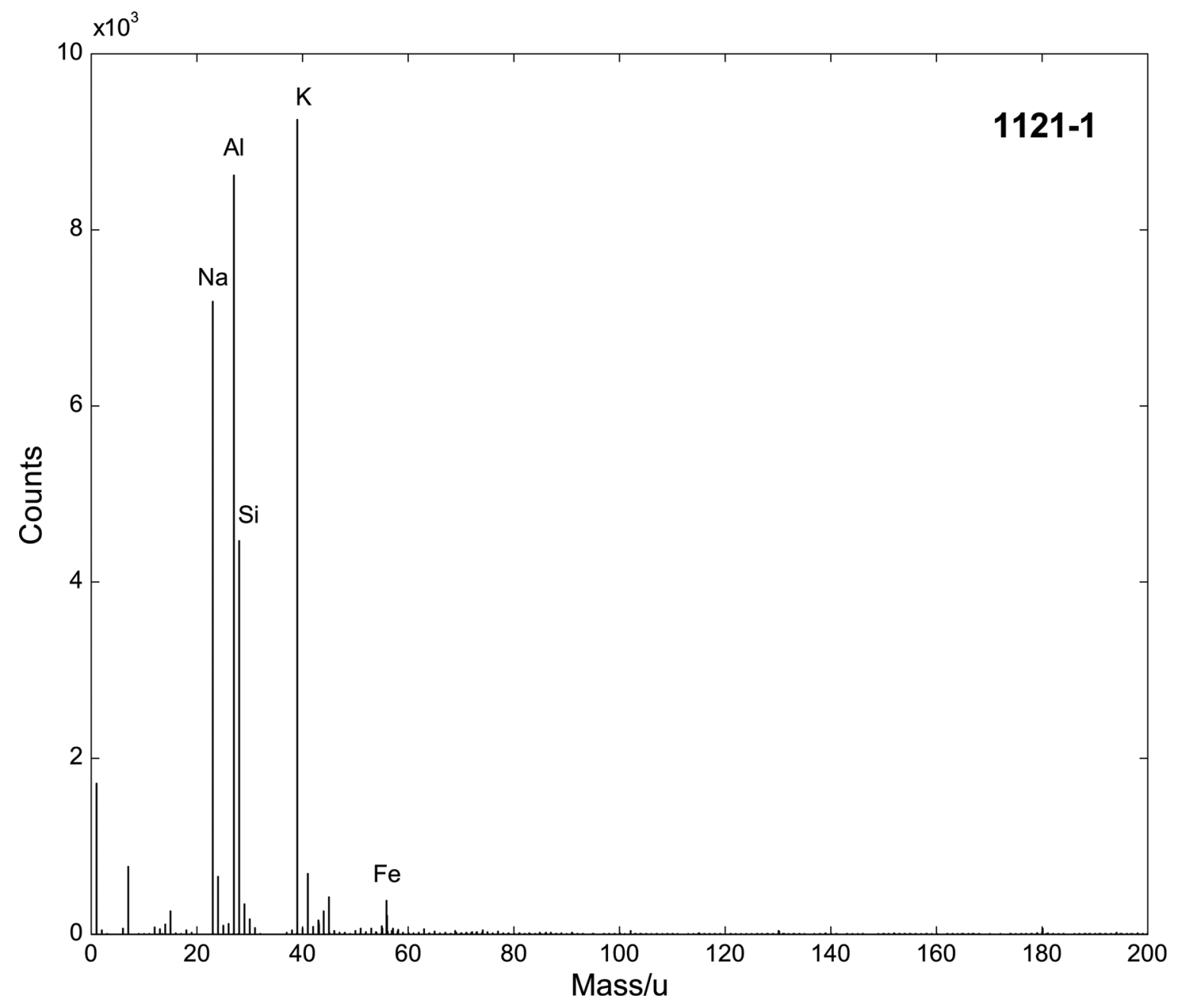

\begin{tabular}{|c|c|}
\hline Accession \# & $01121-01$ \\
\hline Host Material & Coal SS10 \\
\hline Technique & ToF-SIMS \\
\hline Mass Range & $200 \mathrm{Da}$ \\
\hline Instrument & ION-TOF TOF-SIMS IV \\
\hline Analyzer Type & time-of-flight, reflectron \\
\hline Analyzer Mass Resolution & $\sim 5000$ \\
\hline Detector Type & Electron multiplier \\
\hline Specimen Normal to Analyzer & $0^{\circ}$ \\
\hline Primary Beam Ion Gun & ION-TOF \\
\hline Primary Species & $\mathrm{Ga}^{+}$ \\
\hline Primary Ion Pulse Length & $25 \times 10^{-9} \mathrm{sec}$ \\
\hline Primary Ion Pulse Rate & $10 \mathrm{kHz}$ \\
\hline Net Beam Voltage & 25000 \\
\hline Beam Current & $1.5 \mathrm{nA}$ \\
\hline Beam Diameter & $0.1 \mu \mathrm{m}$ \\
\hline Beam Raster Width & $500 \mu \mathrm{m} \times 500 \mu \mathrm{m}$ \\
\hline Beam Incident Angle & $45^{\circ}\left(45^{\circ}\right.$ effective $)$ \\
\hline Source to Analyzer Angle & $45^{\circ}$ \\
\hline Comment & \\
\hline
\end{tabular}




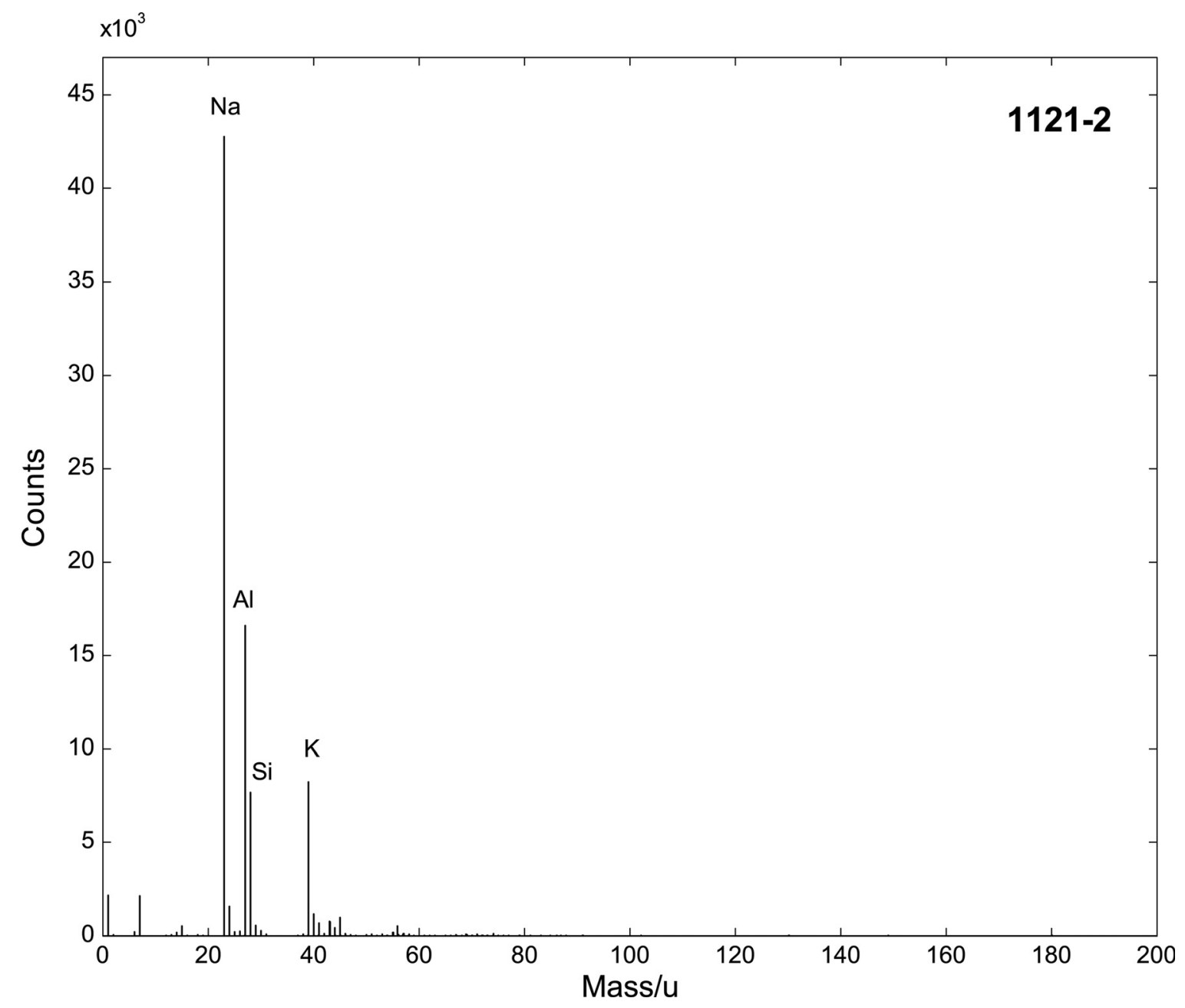

\begin{tabular}{rc}
\hline Accession \# & 01121-02 \\
\hline Host Material & Coal SS10 \\
Technique & ToF-SIMS \\
Mass Range & $200 \mathrm{Da}$ \\
Instrument & ION-TOF TOF-SIMS IV \\
Analyzer Type & time-of-flight, reflectron \\
Detector Type & $\sim 5000$ \\
Analyzer Mass Resolution & Electron multiplier \\
Specimen Normal to Analyzer & $0^{\circ}$ \\
Primary Beam lon Gun & ION-TOF \\
Primary Species & Ga \\
Primary lon Pulse Length & $25 \times 10^{-9}$ sec \\
Primary lon Pulse Rate & $10 \mathrm{kHz}$ \\
Net Beam Voltage & 25000 \\
Beam Current & $1.5 \mathrm{nA}$ \\
Beam Diameter & $0.1 \mu \mathrm{m}$ \\
Beam Raster Width & $500 \mu \mathrm{m} \times 500 \mu \mathrm{m}$ \\
Beam Incident Angle & $45^{\circ}\left(45^{\circ}\right.$ effective $)$ \\
Source to Analyzer Angle & $45^{\circ}$ \\
Comment & \\
\hline
\end{tabular}




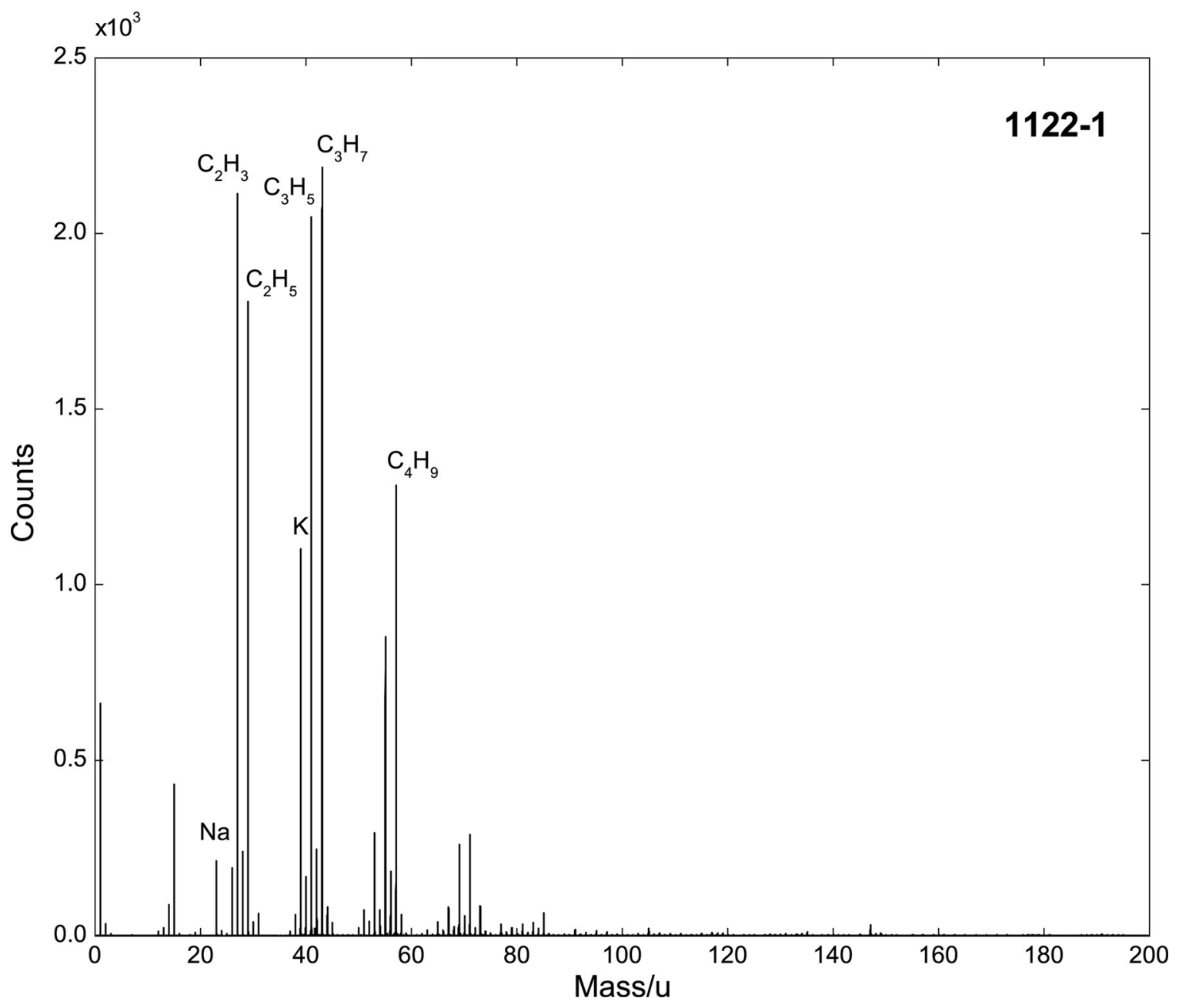

\begin{tabular}{rc}
\hline Accession \# & $\mathbf{0 1 1 2 2 - 0 1}$ \\
\hline Host Material & Coal SS11 \\
Technique & ToF-SIMS \\
Mass Range & 200 Da \\
Instrument & ION-TOF TOF-SIMS IV \\
Analyzer Type & time-of-flight, reflectron \\
Detector Type & $\sim 5000$ \\
Analyzer Mass Resolution & Electron multiplier \\
Specimen Normal to Analyzer & $0^{\circ}$ \\
Primary Beam lon Gun & ION-TOF \\
Primary Species & $\mathrm{Ga}^{+}$ \\
Primary lon Pulse Length & $25 \times 10^{-9} \mathrm{sec}$ \\
Primary lon Pulse Rate & $10 \mathrm{kHz}$ \\
Net Beam Voltage & 25000 \\
Beam Current & $1.5 \mathrm{nA}$ \\
Beam Diameter & $0.1 \mu \mathrm{m}$ \\
Beam Raster Width & $500 \mu \mathrm{m} \times 500 \mu \mathrm{m}$ \\
Beam Incident Angle & $45^{\circ}\left(45^{\circ}\right.$ effective $)$ \\
Source to Analyzer Angle & $45^{\circ}$ \\
Comment & \\
\hline
\end{tabular}




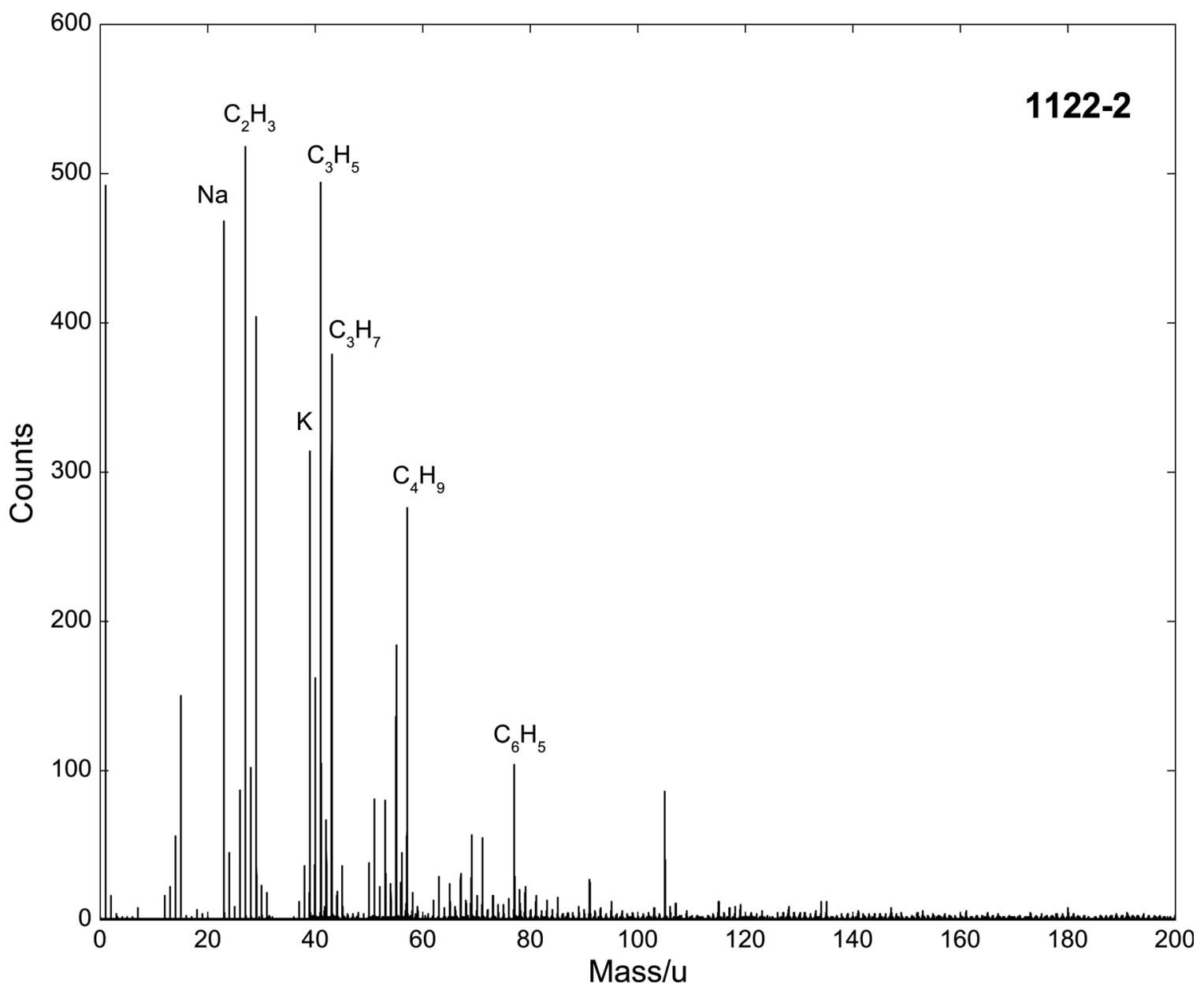

\begin{tabular}{rc}
\hline Accession \# & 01122-02 \\
\hline Host Material & Coal SS11 \\
Technique & ToF-SIMS \\
Mass Range & $200 \mathrm{Da}$ \\
Instrument & ION-TOF TOF-SIMS IV \\
Analyzer Type & time-of-flight, reflectron \\
Detector Type & $\sim 5000$ \\
Analyzer Mass Resolution & Electron multiplier \\
Specimen Normal to Analyzer & $0^{\circ}$ \\
Primary Beam lon Gun & ION-TOF \\
Primary Species & Ga \\
Primary lon Pulse Length & $25 \times 10^{-9} \mathrm{sec}$ \\
Primary lon Pulse Rate & $10 \mathrm{kHz}$ \\
Net Beam Voltage & 25000 \\
Beam Current & $1.5 \mathrm{nA}$ \\
Beam Diameter & $0.1 \mu \mathrm{m}$ \\
Beam Raster Width & $500 \mu \mathrm{m} \times 500 \mu \mathrm{m}$ \\
Beam Incident Angle & $45^{\circ}\left(45^{\circ}\right.$ effective $)$ \\
Source to Analyzer Angle & $45^{\circ}$ \\
Comment & \\
\hline
\end{tabular}




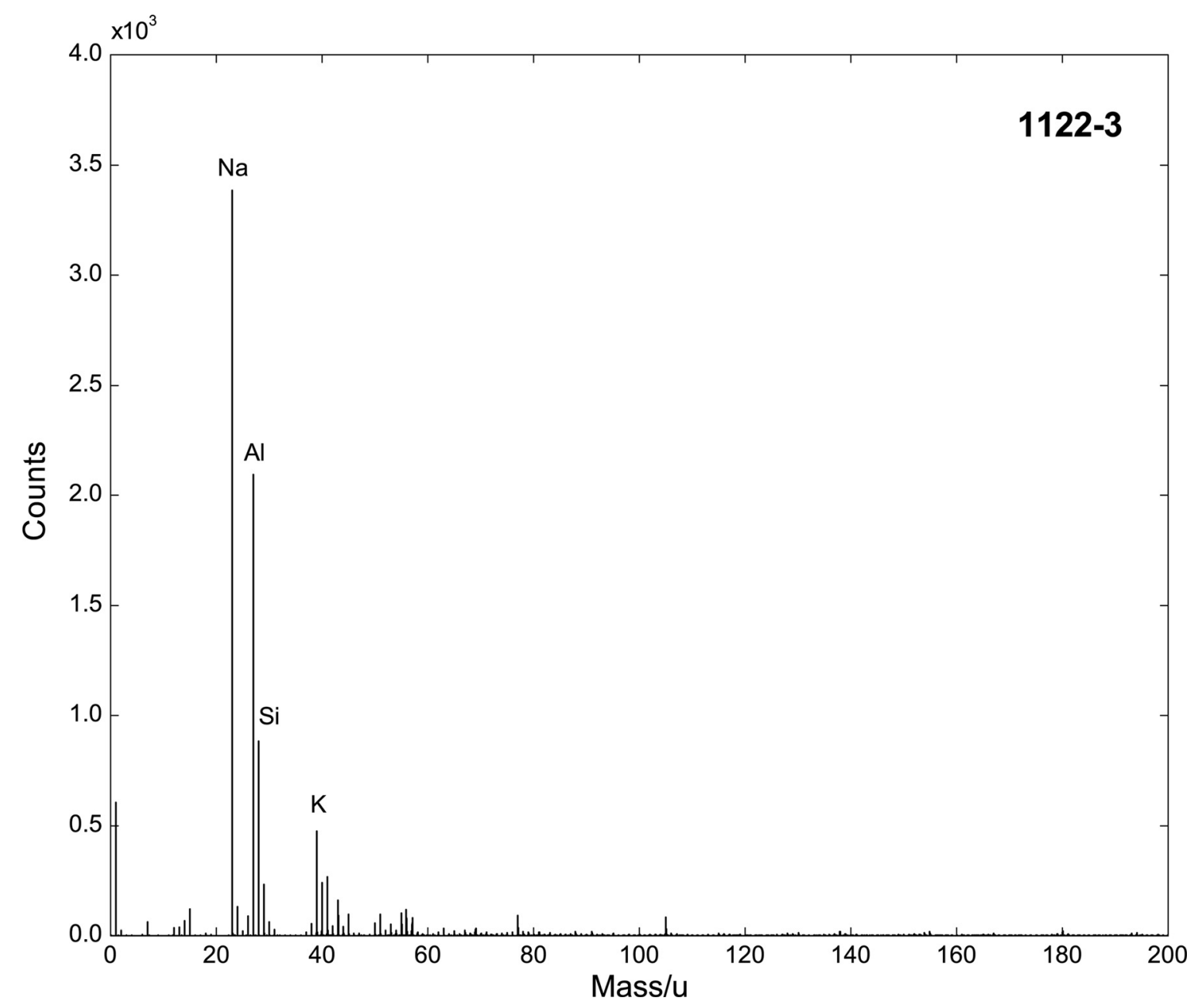

\begin{tabular}{rc}
\hline Accession \# & $\mathbf{0 1 1 2 2 - 0 3}$ \\
\hline Host Material & Coal SS11 \\
Technique & ToF-SIMS \\
Mass Range & $200 \mathrm{Da}$ \\
Instrument & ION-TOF TOF-SIMS IV \\
Analyzer Type & time-of-flight, reflectron \\
Detector Type & $\sim 5000$ \\
Analyzer Mass Resolution & Electron multiplier \\
Specimen Normal to Analyzer & $0^{\circ}$ \\
Primary Beam lon Gun & ION-TOF \\
Primary Species & Ga ${ }^{+}$ \\
Primary lon Pulse Length & $25 \times 10^{-9}$ sec \\
Primary lon Pulse Rate & $10 \mathrm{kHz}$ \\
Net Beam Voltage & 25000 \\
Beam Current & $1.5 \mathrm{nA}$ \\
Beam Diameter & $0.1 \mu \mathrm{m}$ \\
Beam Raster Width & $500 \mu \mathrm{m} \times 500 \mu \mathrm{m}$ \\
Beam Incident Angle & $45^{\circ}\left(45^{\circ}\right.$ effective $)$ \\
Source to Analyzer Angle & $45^{\circ}$ \\
Comment & \\
\hline
\end{tabular}




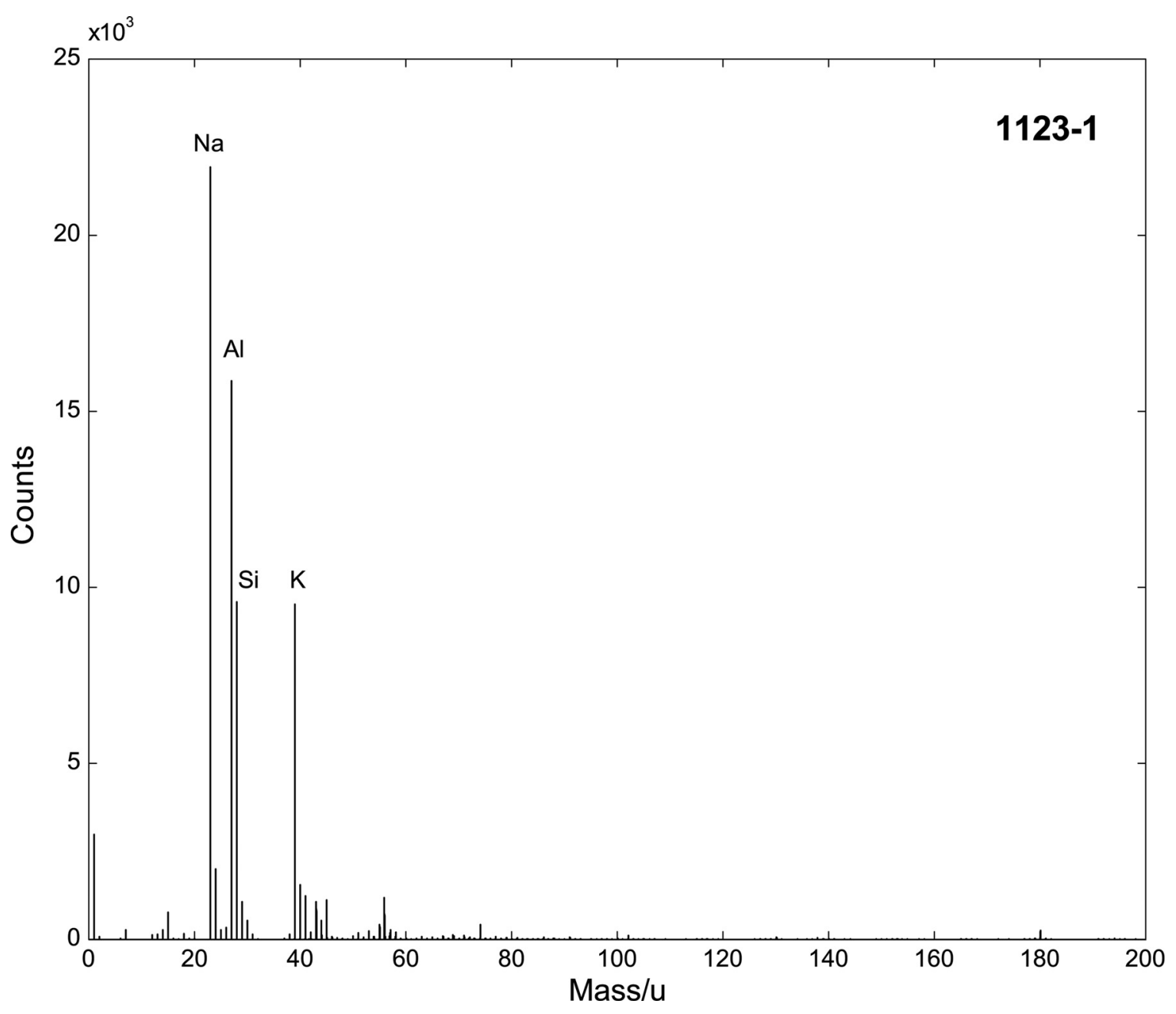

\begin{tabular}{rc}
\hline Accession \# & $\mathbf{0 1 1 2 3 - 0 1}$ \\
\hline Host Material & Coal SS12 \\
Technique & ToF-SIMS \\
Mass Range & $200 \mathrm{Da}$ \\
Instrument & ION-TOF TOF-SIMS IV \\
Analyzer Type & time-of-flight, reflectron \\
Detector Type & $\sim 5000$ \\
Analyzer Mass Resolution & Electron multiplier \\
Specimen Normal to Analyzer & $0^{\circ}$ \\
Primary Beam lon Gun & ION-TOF \\
Primary Species & $\mathrm{Ga}^{+}$ \\
Primary lon Pulse Length & $25 \times 10^{-9} \mathrm{sec}$ \\
Primary lon Pulse Rate & $10 \mathrm{kHz}$ \\
Net Beam Voltage & 25000 \\
Beam Current & $1.5 \mathrm{nA}$ \\
Beam Diameter & $0.1 \mu \mathrm{m}$ \\
Beam Raster Width & $500 \mu \mathrm{m} \times 500 \mu \mathrm{m}$ \\
Beam Incident Angle & $45^{\circ}\left(45^{\circ}\right.$ effective $)$ \\
Source to Analyzer Angle & $45^{\circ}$ \\
Comment & \\
\hline
\end{tabular}




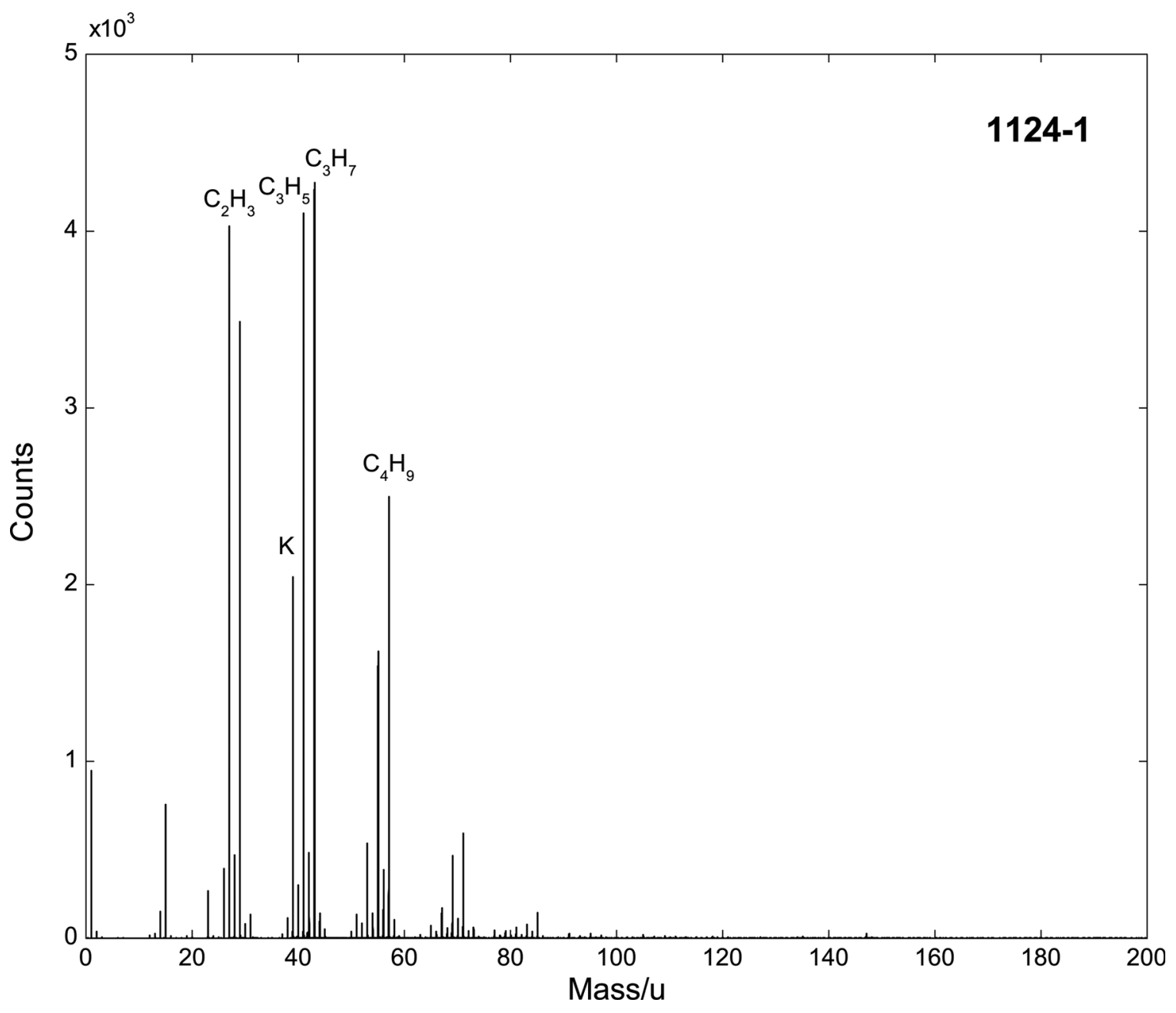

\begin{tabular}{rc}
\hline Accession \# & $\mathbf{0 1 1 2 4 - 0 1}$ \\
\hline Host Material & Coal SS13 \\
Technique & ToF-SIMS \\
Mass Range & $200 \mathrm{Da}$ \\
Instrument & ION-TOF TOF-SIMS IV \\
Analyzer Type & time-of-flight, reflectron \\
Detector Type & $\sim 5000$ \\
Analyzer Mass Resolution & Electron multiplier \\
Specimen Normal to Analyzer & $0^{\circ}$ \\
Primary Beam lon Gun & ON-TOF \\
Primary Species & Ga \\
Primary lon Pulse Length & $25 \times 10^{-9} \mathrm{sec}$ \\
Primary lon Pulse Rate & $10 \mathrm{kHz}$ \\
Net Beam Voltage & 25000 \\
Beam Current & $1.5 \mathrm{nA}$ \\
Beam Diameter & $0.1 \mu \mathrm{m}$ \\
Beam Raster Width & $500 \mu \mathrm{m} \times 500 \mu \mathrm{m}$ \\
Beam Incident Angle & $45^{\circ}\left(45^{\circ}\right.$ effective $)$ \\
Source to Analyzer Angle & $45^{\circ}$ \\
Comment & \\
\hline
\end{tabular}




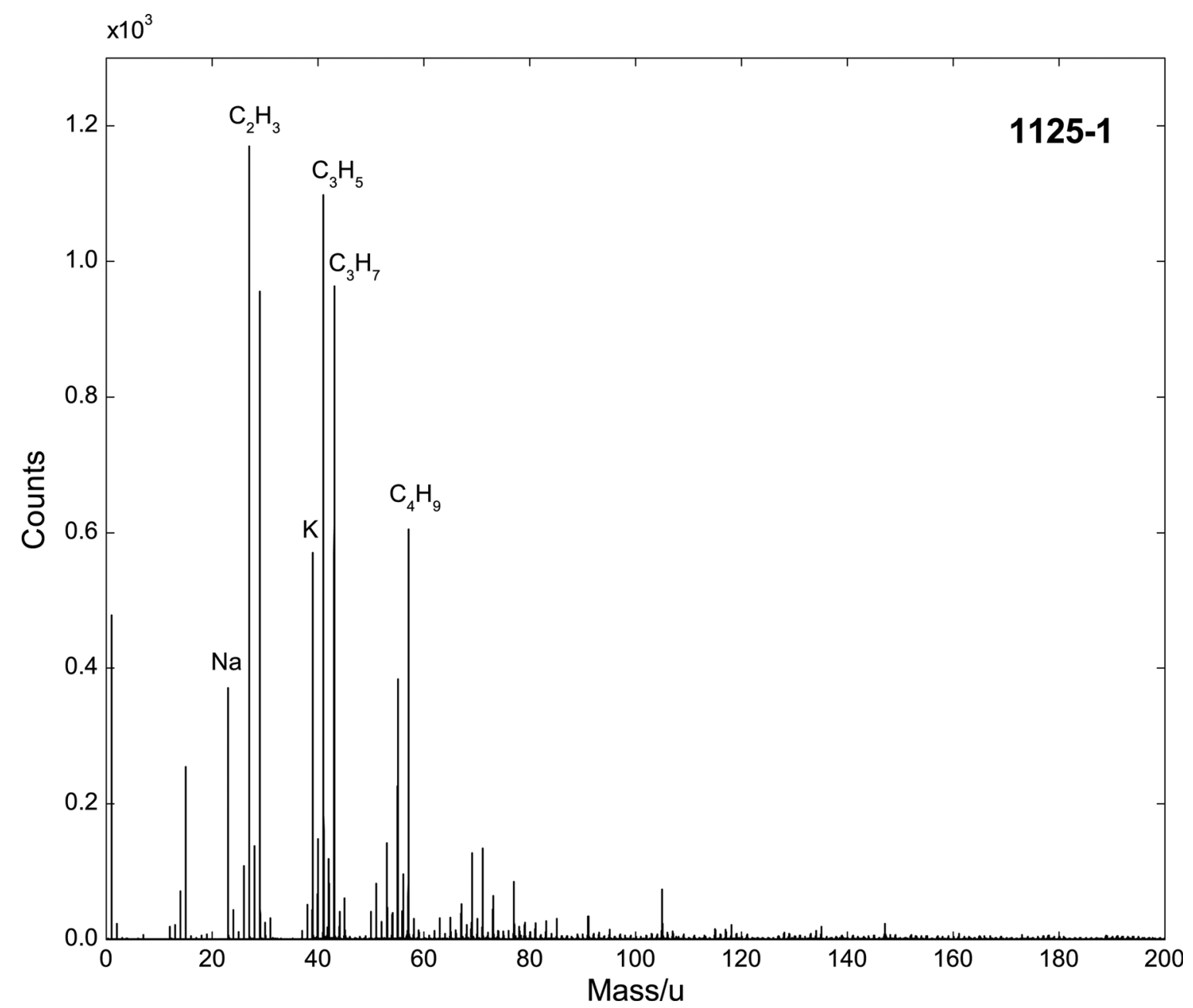

\begin{tabular}{rc}
\hline Accession \# & 01125-01 \\
\hline Host Material & Coal SS14 \\
Technique & ToF-SIMS \\
Mass Range & $200 \mathrm{Da}$ \\
Instrument & ION-TOF TOF-SIMS IV \\
Analyzer Type & time-of-flight, reflectron \\
Detector Type & $\sim 5000$ \\
Analyzer Mass Resolution & Electron multiplier \\
Specimen Normal to Analyzer & $0^{\circ}$ \\
Primary Beam lon Gun & ION-TOF \\
Primary Species & Ga \\
Primary lon Pulse Length & $25 \times 10^{-9}$ sec \\
Primary lon Pulse Rate & $10 \mathrm{kHz}$ \\
Net Beam Voltage & 25000 \\
Beam Current & $1.5 \mathrm{nA}$ \\
Beam Diameter & $0.1 \mu \mathrm{m}$ \\
Beam Raster Width & $500 \mu \mathrm{m} \times 500 \mu \mathrm{m}$ \\
Beam Incident Angle & $45^{\circ}\left(45^{\circ}\right.$ effective $)$ \\
Source to Analyzer Angle & $45^{\circ}$ \\
Comment & \\
\hline
\end{tabular}




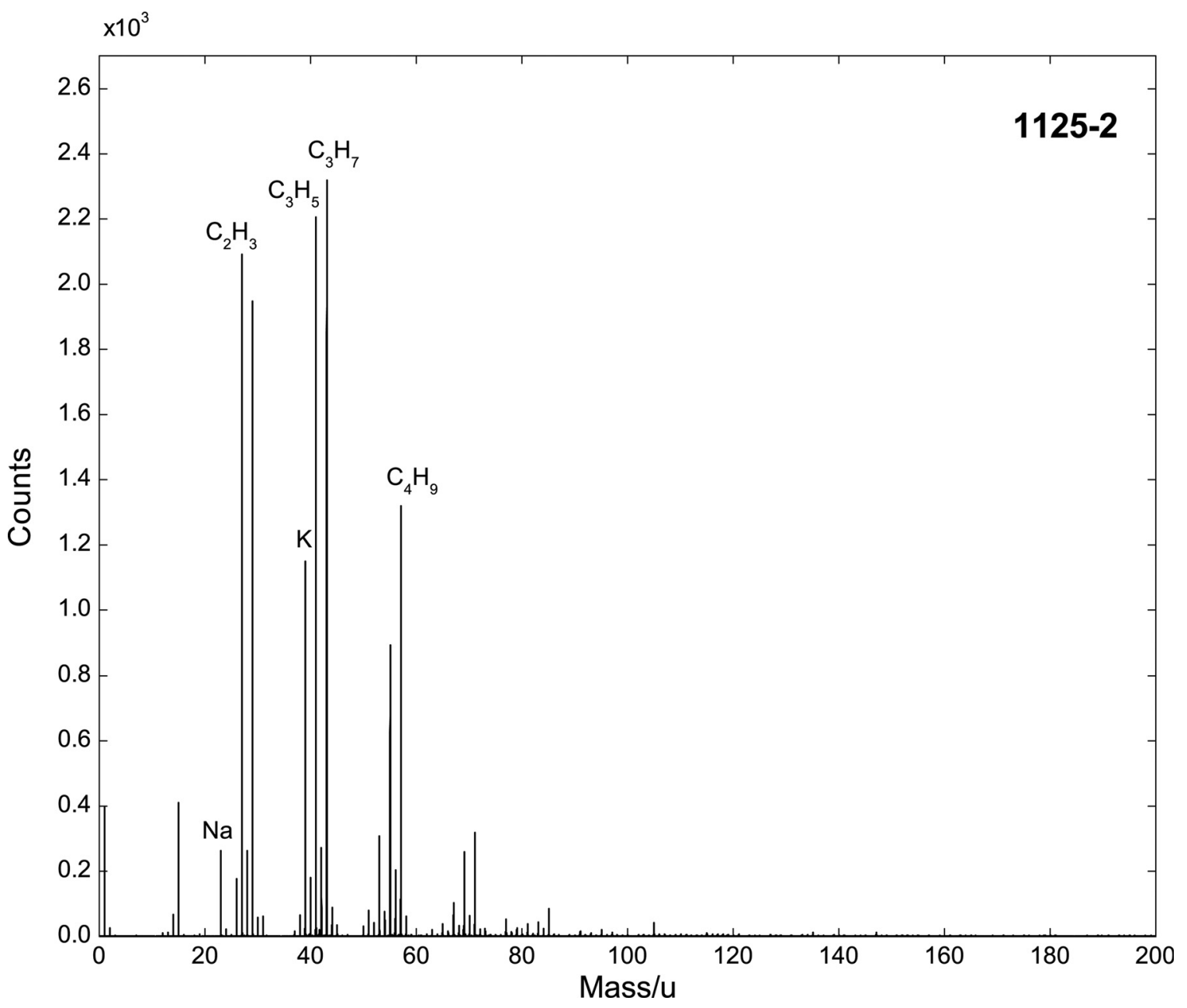

\begin{tabular}{rc}
\hline Accession \# & 01125-02 \\
\hline Host Material & Coal SS14 \\
Technique & ToF-SIMS \\
Mass Range & 200 Da \\
Instrument & ION-TOF TOF-SIMS IV \\
Analyzer Type & time-of-flight, reflectron \\
Detector Type & $\sim 5000$ \\
Analyzer Mass Resolution & Electron multiplier \\
Specimen Normal to Analyzer & $0^{\circ}$ \\
Primary Beam lon Gun & ION-TOF \\
Primary Species & Ga \\
Primary lon Pulse Length & $25 \times 10^{-9} \mathrm{sec}$ \\
Primary lon Pulse Rate & $10 \mathrm{kHz}$ \\
Net Beam Voltage & 25000 \\
Beam Current & $1.5 \mathrm{nA}$ \\
Beam Diameter & $0.1 \mu \mathrm{m}$ \\
Beam Raster Width & $500 \mu \mathrm{m} \times 500 \mu \mathrm{m}$ \\
Beam Incident Angle & $45^{\circ}\left(45^{\circ}\right.$ effective $)$ \\
Source to Analyzer Angle & $45^{\circ}$ \\
Comment & \\
\hline
\end{tabular}




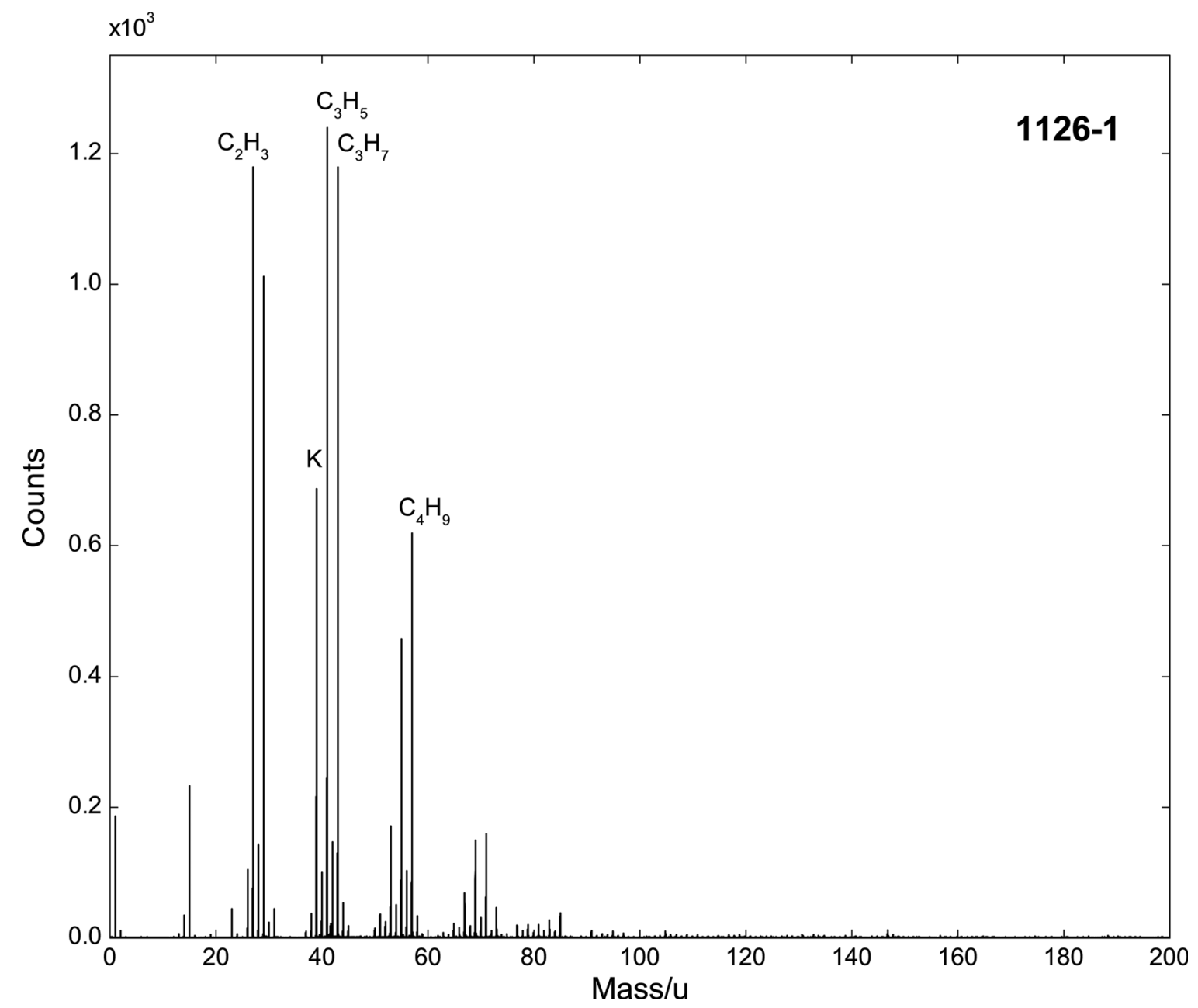

\begin{tabular}{rc}
\hline Accession \# & $\mathbf{0 1 1 2 6 - 0 1}$ \\
\hline Host Material & Coal SS15 \\
Technique & ToF-SIMS \\
Mass Range & $200 \mathrm{Da}$ \\
Instrument & ION-TOF TOF-SIMS IV \\
Analyzer Type & time-of-flight, reflectron \\
Detector Type & $\sim 5000$ \\
Analyzer Mass Resolution & Electron multiplier \\
Specimen Normal to Analyzer & $0^{\circ}$ \\
Primary Beam lon Gun & ION-TOF \\
Primary Species & $\mathrm{Ga}{ }^{+}$ \\
Primary lon Pulse Length & $25 \times 10^{-9} \mathrm{sec}$ \\
Primary lon Pulse Rate & $10 \mathrm{kHz}$ \\
Net Beam Voltage & 25000 \\
Beam Current & $1.5 \mathrm{nA}$ \\
Beam Diameter & $0.1 \mu \mathrm{m}$ \\
Beam Raster Width & $500 \mu \mathrm{m} \times 500 \mu \mathrm{m}$ \\
Beam Incident Angle & $45^{\circ}\left(45^{\circ}\right.$ effective $)$ \\
Source to Analyzer Angle & $45^{\circ}$ \\
Comment & \\
\hline
\end{tabular}




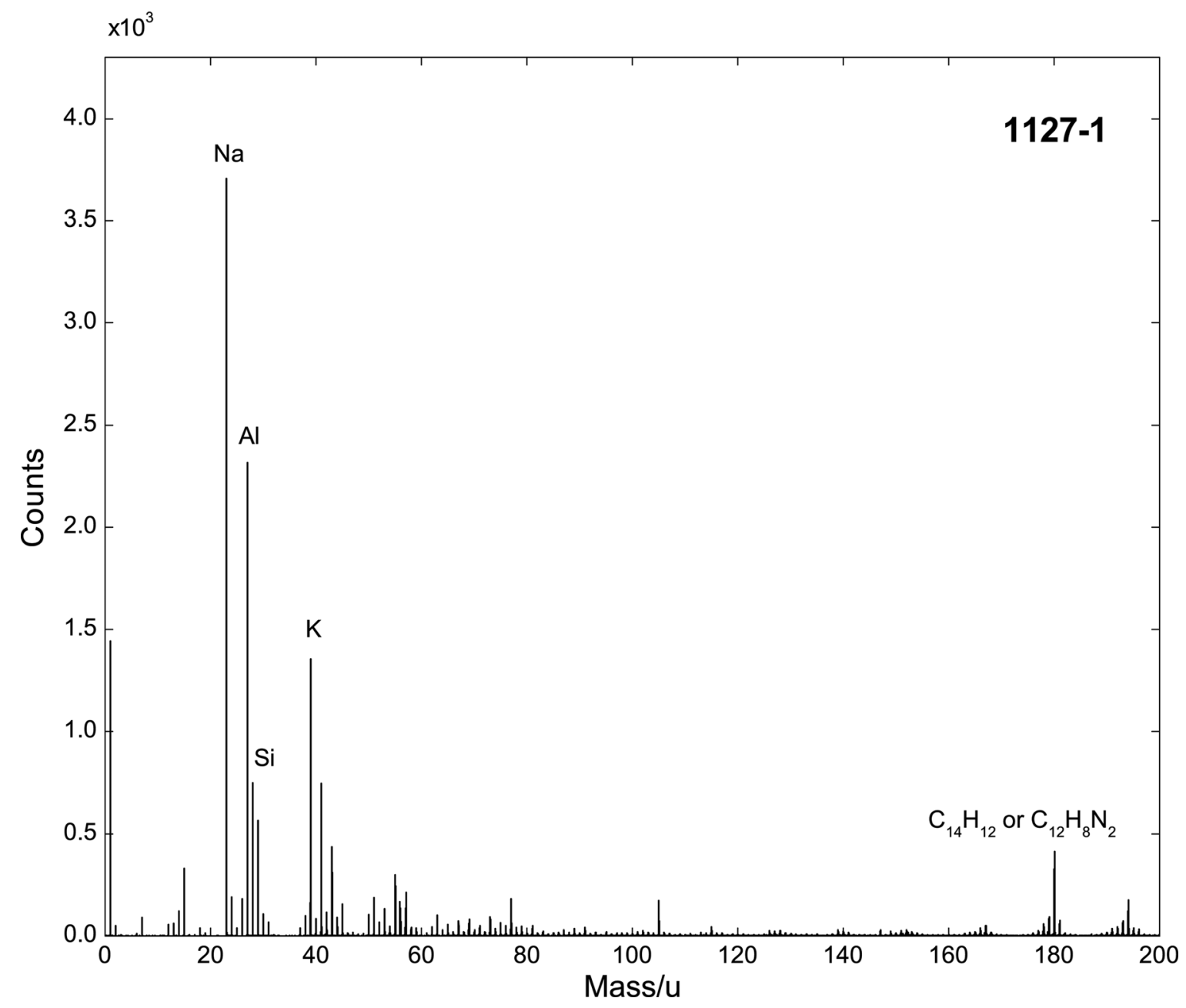

\begin{tabular}{rc}
\hline Accession \# & $\mathbf{0 1 1 2 7 - 0 1}$ \\
\hline Host Material & Coal SS16 \\
Technique & ToF-SIMS \\
Mass Range & $200 \mathrm{Da}$ \\
Instrument & ION-TOF TOF-SIMS IV \\
Analyzer Type & time-of-flight, reflectron \\
Detector Type & $\sim 5000$ \\
Analyzer Mass Resolution & Electron multiplier \\
Specimen Normal to Analyzer & $0^{\circ}$ \\
Primary Beam lon Gun & ION-TOF \\
Primary Species & $\mathrm{Ga}^{+}$ \\
Primary lon Pulse Length & $25 \times 10^{-9} \mathrm{sec}$ \\
Primary lon Pulse Rate & $10 \mathrm{kHz}$ \\
Net Beam Voltage & 25000 \\
Beam Current & $1.5 \mathrm{nA}$ \\
Beam Diameter & $0.1 \mu \mathrm{m}$ \\
Beam Raster Width & $500 \mu \mathrm{m} \times 500 \mu \mathrm{m}$ \\
Beam Incident Angle & $45^{\circ}\left(45^{\circ}\right.$ effective $)$ \\
Source to Analyzer Angle & $45^{\circ}$ \\
Comment & \\
\hline
\end{tabular}




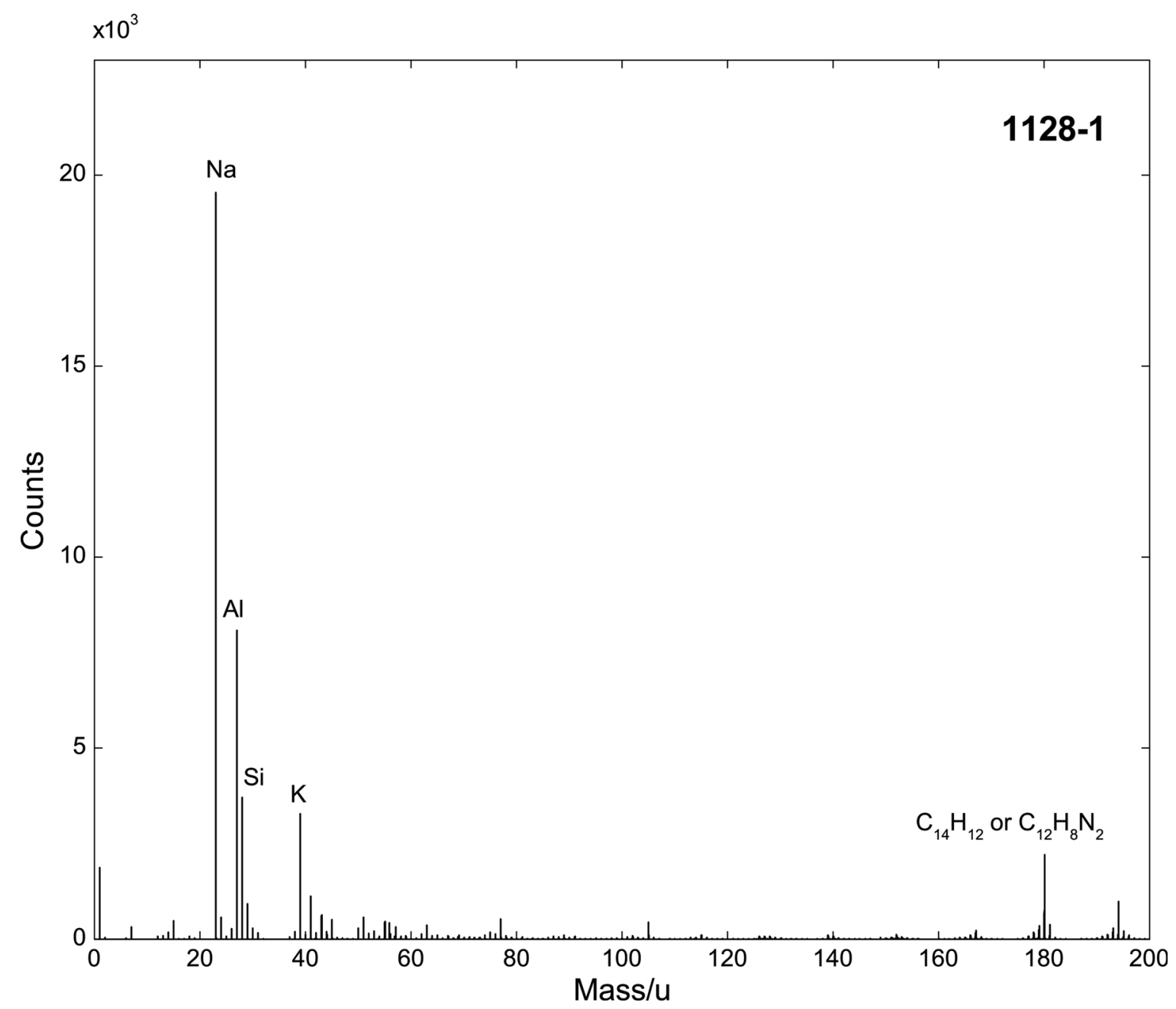

\begin{tabular}{rc}
\hline Accession \# & $\mathbf{0 1 1 2 8 - 0 1}$ \\
\hline Host Material & Coal SS17 \\
Technique & ToF-SIMS \\
Mass Range & 200 Da \\
Instrument & ION-TOF TOF-SIMS IV \\
Analyzer Type & time-of-flight, reflectron \\
Detector Type & $\sim 5000$ \\
Analyzer Mass Resolution & Electron multiplier \\
Specimen Normal to Analyzer & $0^{\circ}$ \\
Primary Beam lon Gun & ION-TOF \\
Primary Species & Ga ${ }^{+}$ \\
Primary lon Pulse Length & $25 \times 10^{-9} \mathrm{sec}$ \\
Primary lon Pulse Rate & $10 \mathrm{kHz}$ \\
Net Beam Voltage & 25000 \\
Beam Current & $1.5 \mathrm{nA}$ \\
Beam Diameter & $0.1 \mu \mathrm{m}$ \\
Beam Raster Width & $500 \mu \mathrm{m} \times 500 \mu \mathrm{m}$ \\
Beam Incident Angle & $45^{\circ}\left(45^{\circ}\right.$ effective $)$ \\
Source to Analyzer Angle & $45^{\circ}$ \\
Comment & \\
\hline
\end{tabular}




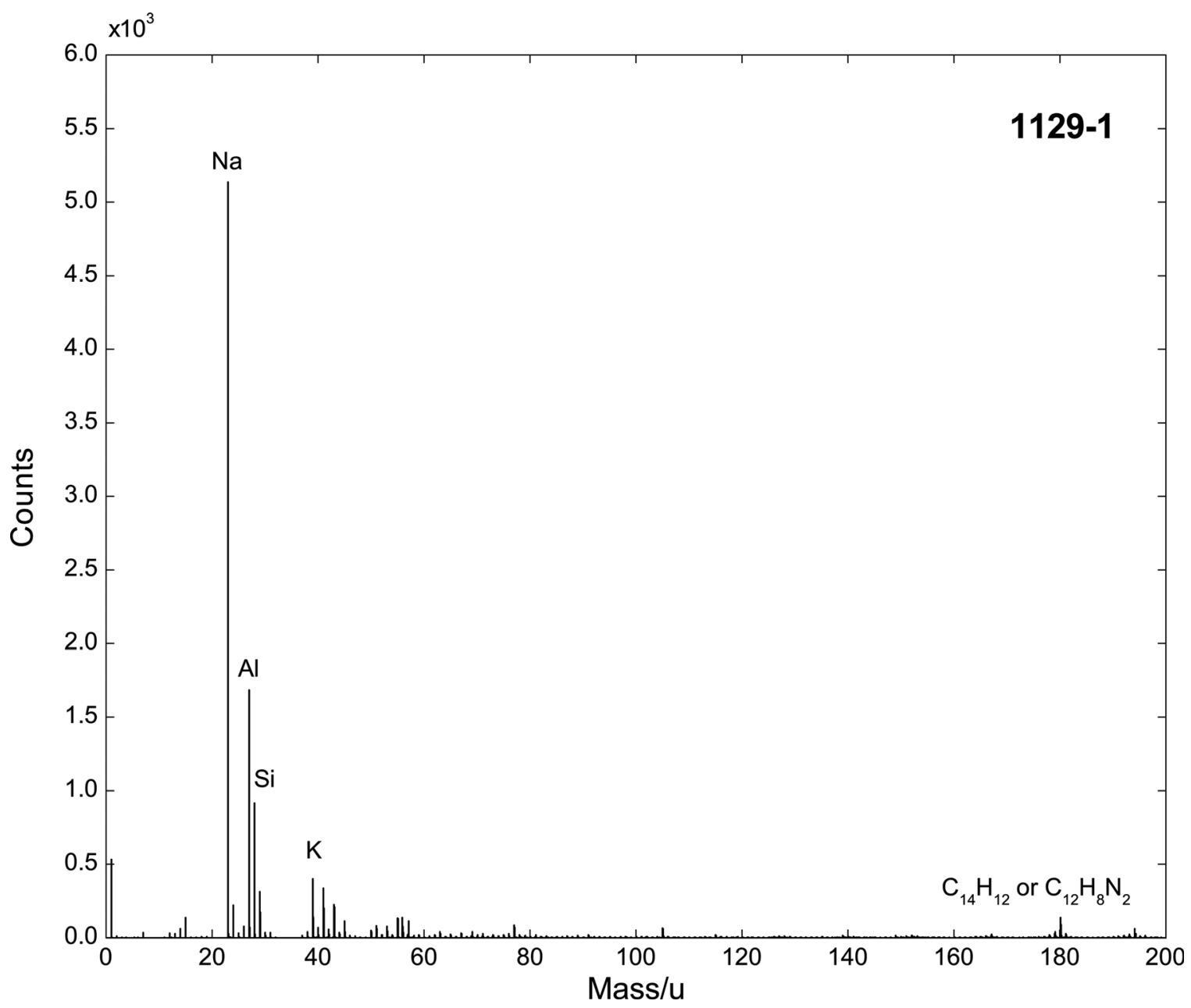

\begin{tabular}{|c|c|}
\hline Accession \# & 01129-01 \\
\hline Host Material & Coal SS18 \\
\hline Technique & ToF-SIMS \\
\hline Mass Range & $200 \mathrm{Da}$ \\
\hline Instrument & ION-TOF TOF-SIMS IV \\
\hline Analyzer Type & time-of-flight, reflectron \\
\hline Analyzer Mass Resolution & $\sim 5000$ \\
\hline Detector Type & Electron multiplier \\
\hline Specimen Normal to Analyzer & $0^{\circ}$ \\
\hline Primary Beam Ion Gun & ION-TOF \\
\hline Primary Species & $\mathrm{Ga}^{+}$ \\
\hline Primary Ion Pulse Length & $25 \times 10^{-9} \mathrm{sec}$ \\
\hline Primary Ion Pulse Rate & $10 \mathrm{kHz}$ \\
\hline Net Beam Voltage & 25000 \\
\hline Beam Current & $1.5 \mathrm{nA}$ \\
\hline Beam Diameter & $0.1 \mu \mathrm{m}$ \\
\hline Beam Raster Width & $500 \mu \mathrm{m} \times 500 \mu \mathrm{m}$ \\
\hline Beam Incident Angle & $45^{\circ}$ (45 effective) \\
\hline Source to Analyzer Angle & $45^{\circ}$ \\
\hline Comment & \\
\hline
\end{tabular}




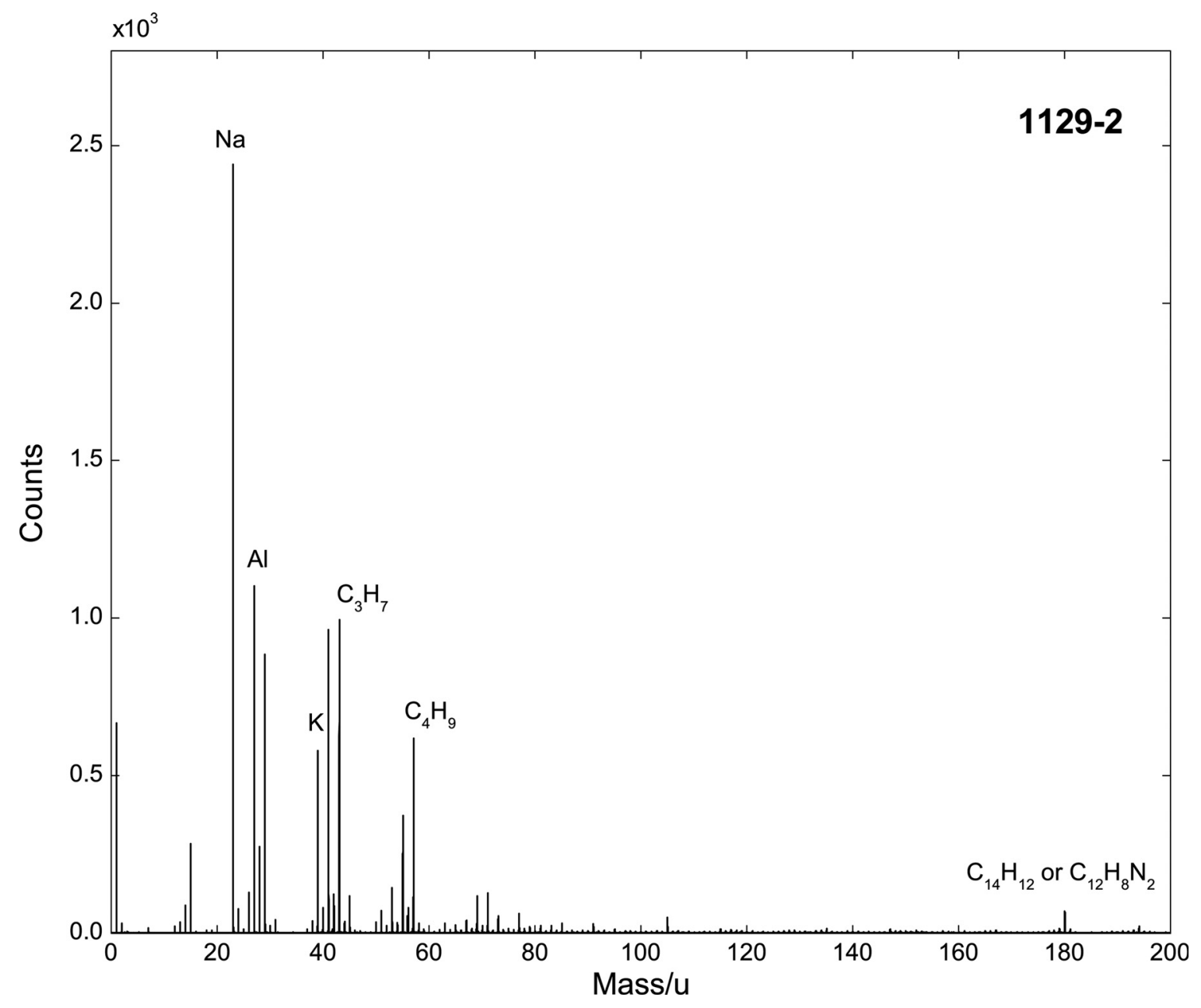

\begin{tabular}{rc}
\hline Accession \# & $\mathbf{0 1 1 2 9 - 0 2}$ \\
\hline Host Material & Coal SS18 \\
Technique & ToF-SIMS \\
Mass Range & $200 \mathrm{Da}$ \\
Instrument & ION-TOF TOF-SIMS IV \\
Analyzer Type & time-of-flight, reflectron \\
Detector Type & $\sim 5000$ \\
Analyzer Mass Resolution & Electron multiplier \\
Specimen Normal to Analyzer & $0^{\circ}$ \\
Primary Beam lon Gun & ION-TOF \\
Primary Species & $\mathrm{Ga}^{+}$ \\
Primary lon Pulse Length & $25 \times 10^{-9} \mathrm{sec}$ \\
Primary lon Pulse Rate & $10 \mathrm{kHz}$ \\
Net Beam Voltage & 25000 \\
Beam Current & $1.5 \mathrm{nA}$ \\
Beam Diameter & $0.1 \mu \mathrm{m}$ \\
Beam Raster Width & $500 \mu \mathrm{m} \times 500 \mu \mathrm{m}$ \\
Beam Incident Angle & $45^{\circ}\left(45^{\circ}\right.$ effective $)$ \\
Source to Analyzer Angle & $45^{\circ}$ \\
Comment & \\
\hline
\end{tabular}




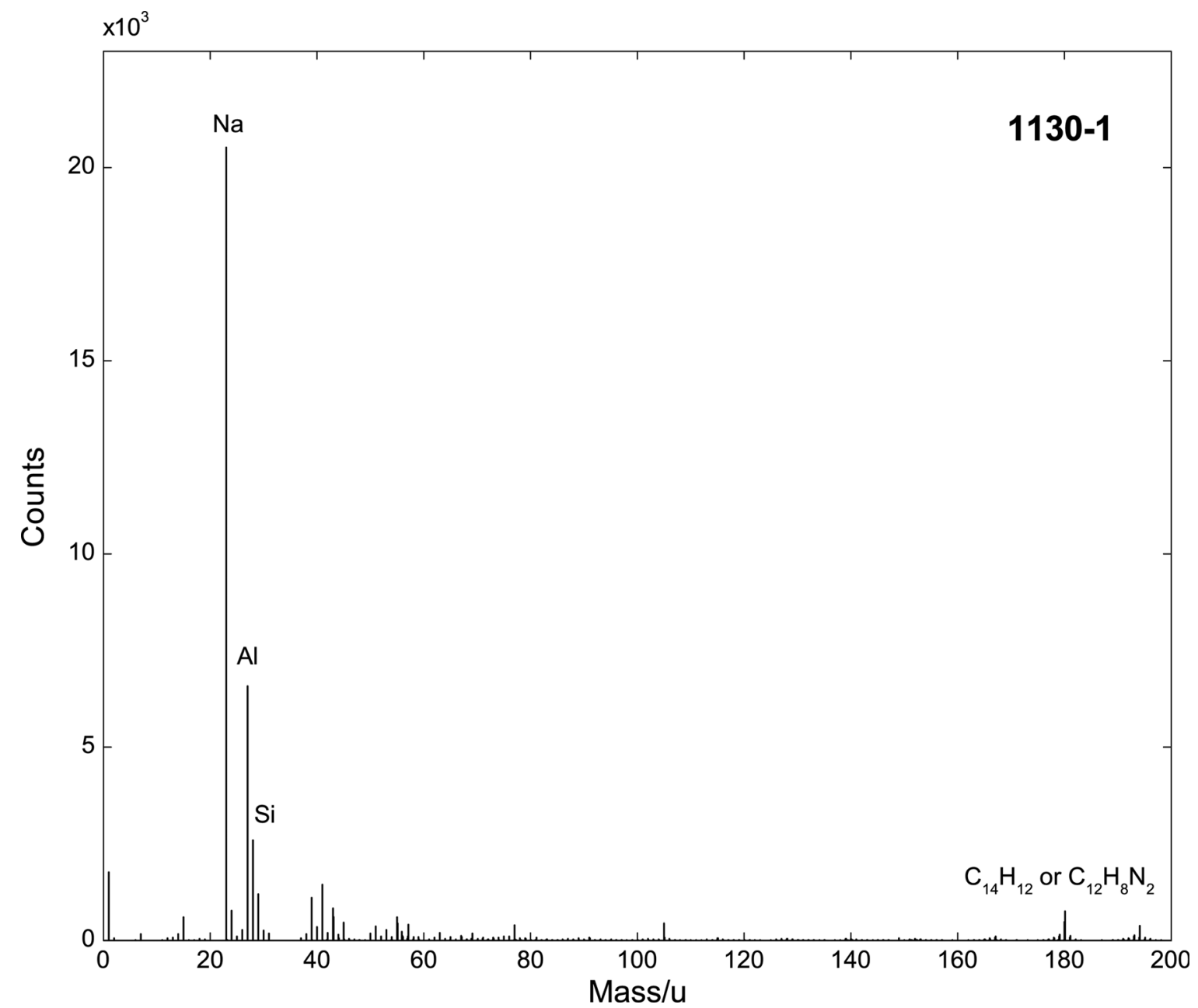

\begin{tabular}{rc}
\hline Accession \# & $\mathbf{0 1 1 3 0 - 0 1}$ \\
\hline Host Material & Coal SS19 \\
Technique & ToF-SIMS \\
Mass Range & $200 \mathrm{Da}$ \\
Instrument & ION-TOF TOF-SIMS IV \\
Analyzer Type & time-of-flight, reflectron \\
Detector Type & $\sim 5000$ \\
Analyzer Mass Resolution & Electron multiplier \\
Specimen Normal to Analyzer & $0^{\circ}$ \\
Primary Beam lon Gun & ION-TOF \\
Primary Species & Ga \\
Primary lon Pulse Length & $25 \times 10^{-9}$ sec \\
Primary lon Pulse Rate & $10 \mathrm{kHz}$ \\
Net Beam Voltage & 25000 \\
Beam Current & $1.5 \mathrm{nA}$ \\
Beam Diameter & $0.1 \mu \mathrm{m}$ \\
Beam Raster Width & $500 \mu \mathrm{m} \times 500 \mu \mathrm{m}$ \\
Beam Incident Angle & $45^{\circ}\left(45^{\circ}\right.$ effective $)$ \\
Source to Analyzer Angle & $45^{\circ}$ \\
Comment & \\
\hline
\end{tabular}




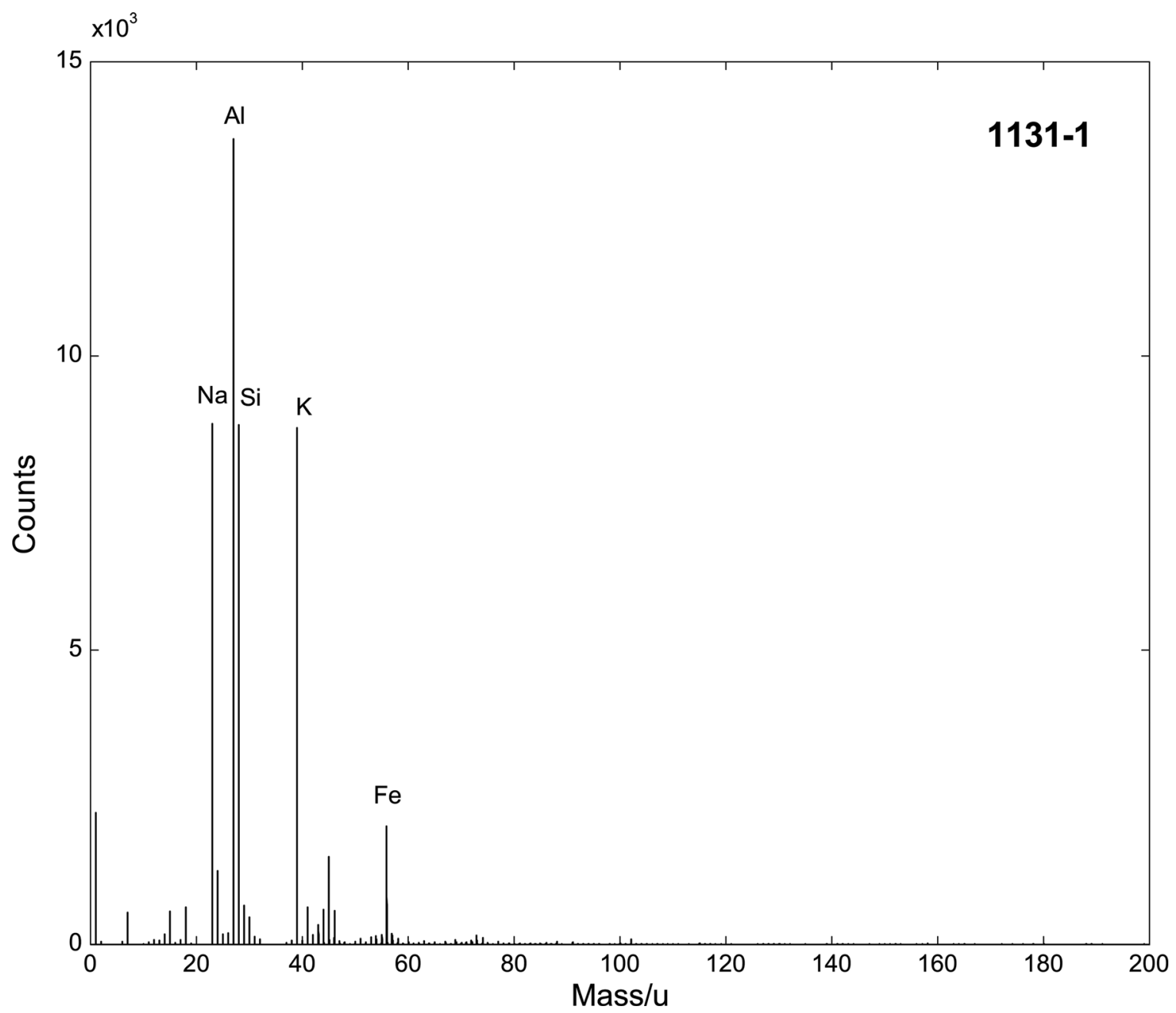

\begin{tabular}{|c|c|}
\hline Accession \# & $01131-01$ \\
\hline Host Material & Coal Blackthunder \\
\hline Technique & ToF-SIMS \\
\hline Mass Range & $200 \mathrm{Da}$ \\
\hline Instrument & ION-TOF TOF-SIMS IV \\
\hline Analyzer Type & time-of-flight, reflectron \\
\hline Analyzer Mass Resolution & $\sim 5000$ \\
\hline Detector Type & Electron multiplier \\
\hline Specimen Normal to Analyzer & $0^{\circ}$ \\
\hline Primary Beam Ion Gun & ION-TOF \\
\hline Primary Species & $\mathrm{Ga}^{+}$ \\
\hline Primary Ion Pulse Length & $25 \times 10^{-9} \mathrm{sec}$ \\
\hline Primary Ion Pulse Rate & $10 \mathrm{kHz}$ \\
\hline Net Beam Voltage & 25000 \\
\hline Beam Current & $1.5 \mathrm{nA}$ \\
\hline Beam Diameter & $0.1 \mu \mathrm{m}$ \\
\hline Beam Raster Width & $500 \mu \mathrm{m} \times 500 \mu \mathrm{m}$ \\
\hline Beam Incident Angle & $45^{\circ}\left(45^{\circ}\right.$ effective $)$ \\
\hline Source to Analyzer Angle & $45^{\circ}$ \\
\hline Comment & \\
\hline
\end{tabular}




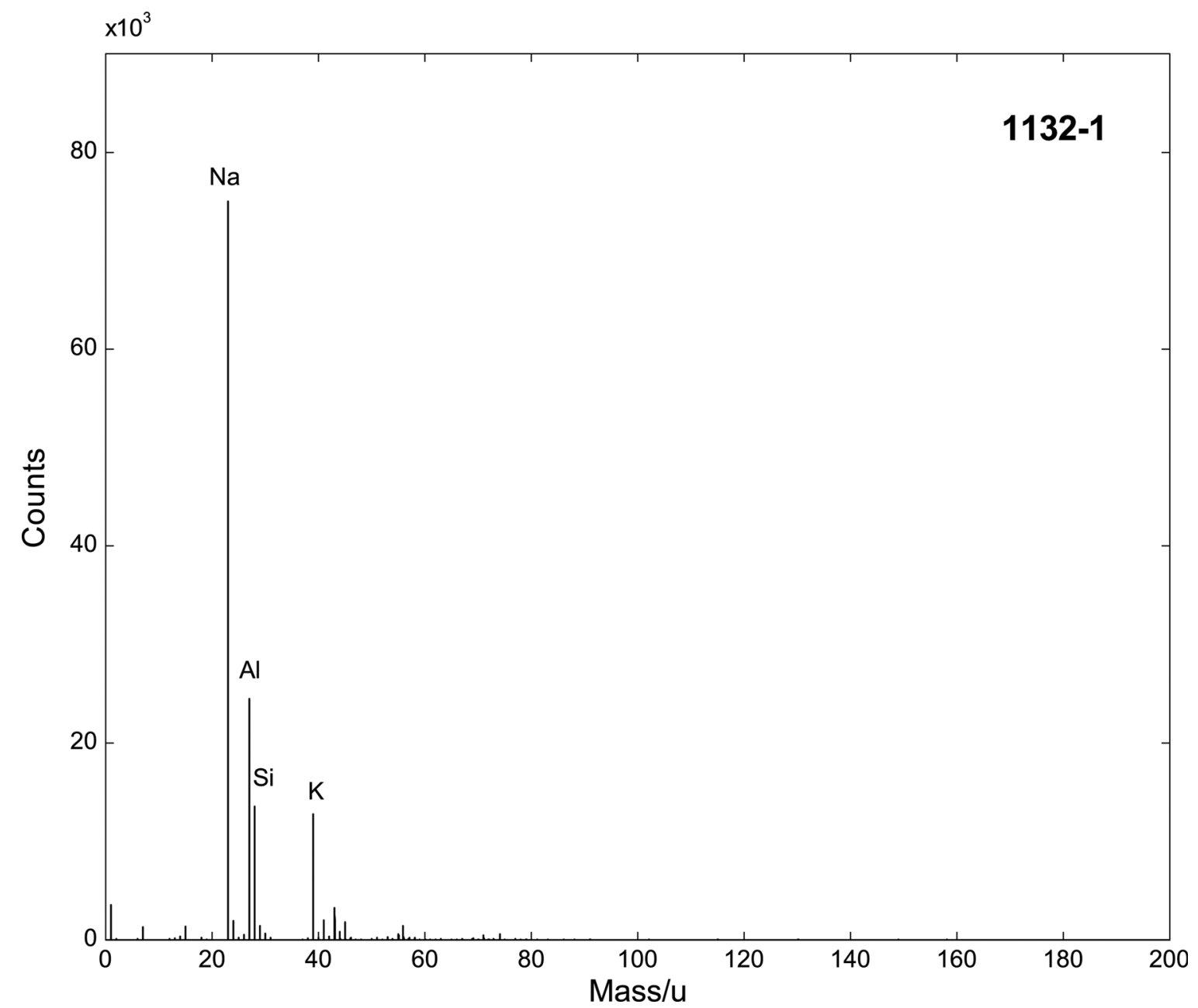

\begin{tabular}{rc}
\hline Accession \# & 01132-01 \\
\hline Host Material & Coal CJR012 \\
Technique & ToF-SIMS \\
Mass Range & 200 Da \\
Instrument & ION-TOF TOF-SIMS IV \\
Analyzer Type & time-of-flight, reflectron \\
Detector Type & $\sim 5000$ \\
Analyzer Mass Resolution & Electron multiplier \\
Specimen Normal to Analyzer & $0^{\circ}$ \\
Primary Beam lon Gun & ION-TOF \\
Primary Species & Ga \\
Primary lon Pulse Length & $25 \times 10^{-9} \mathrm{sec}$ \\
Primary lon Pulse Rate & $10 \mathrm{kHz}$ \\
Net Beam Voltage & 25000 \\
Beam Current & $1.5 \mathrm{nA}$ \\
Beam Diameter & $0.1 \mu \mathrm{m}$ \\
Beam Raster Width & $500 \mu \mathrm{m} \times 500 \mu \mathrm{m}$ \\
Beam Incident Angle & $45^{\circ}\left(45^{\circ}\right.$ effective $)$ \\
Source to Analyzer Angle & $45^{\circ}$ \\
Comment & \\
\hline
\end{tabular}




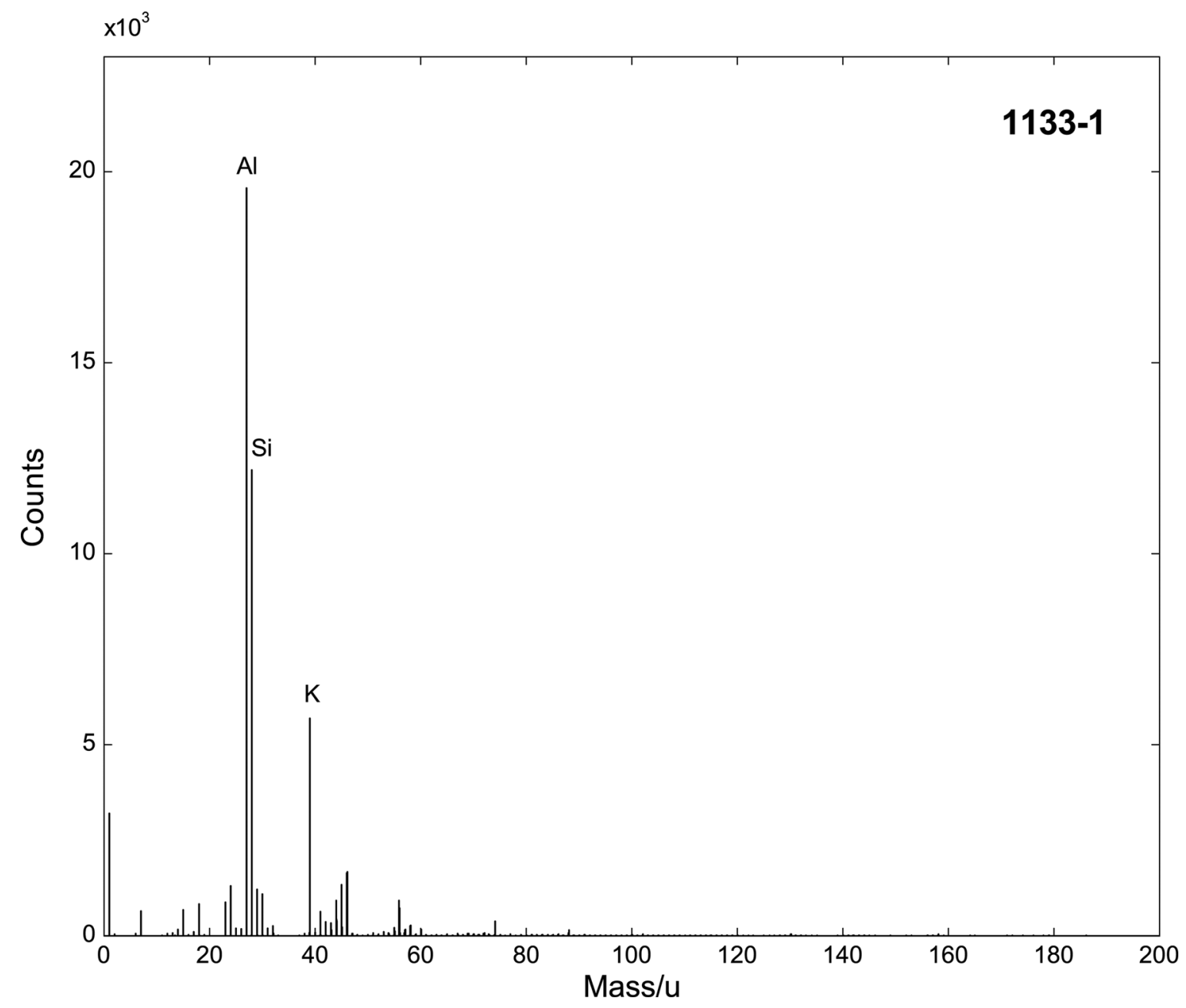

\begin{tabular}{rc}
\hline Accession \# & 01133-01 \\
\hline Host Material & Coal GAT034 \\
Technique & ToF-SIMS \\
Mass Range & 200 Da \\
Instrument & ION-TOF TOF-SIMS IV \\
Analyzer Type & time-of-flight, reflectron \\
Detector Type & $\sim 5000$ \\
Analyzer Mass Resolution & Electron multiplier \\
Specimen Normal to Analyzer & $0^{\circ}$ \\
Primary Beam lon Gun & ION-TOF \\
Primary Species & Ga \\
Primary lon Pulse Length & $25 \times 10^{-9}$ sec \\
Primary lon Pulse Rate & $10 \mathrm{kHz}$ \\
Net Beam Voltage & 25000 \\
Beam Current & $1.5 \mathrm{nA}$ \\
Beam Diameter & $0.1 \mu \mathrm{m}$ \\
Beam Raster Width & $500 \mu \mathrm{m} \times 500 \mu \mathrm{m}$ \\
Beam Incident Angle & $45^{\circ}\left(45^{\circ}\right.$ effective $)$ \\
Source to Analyzer Angle & $45^{\circ}$ \\
Comment & \\
\hline
\end{tabular}




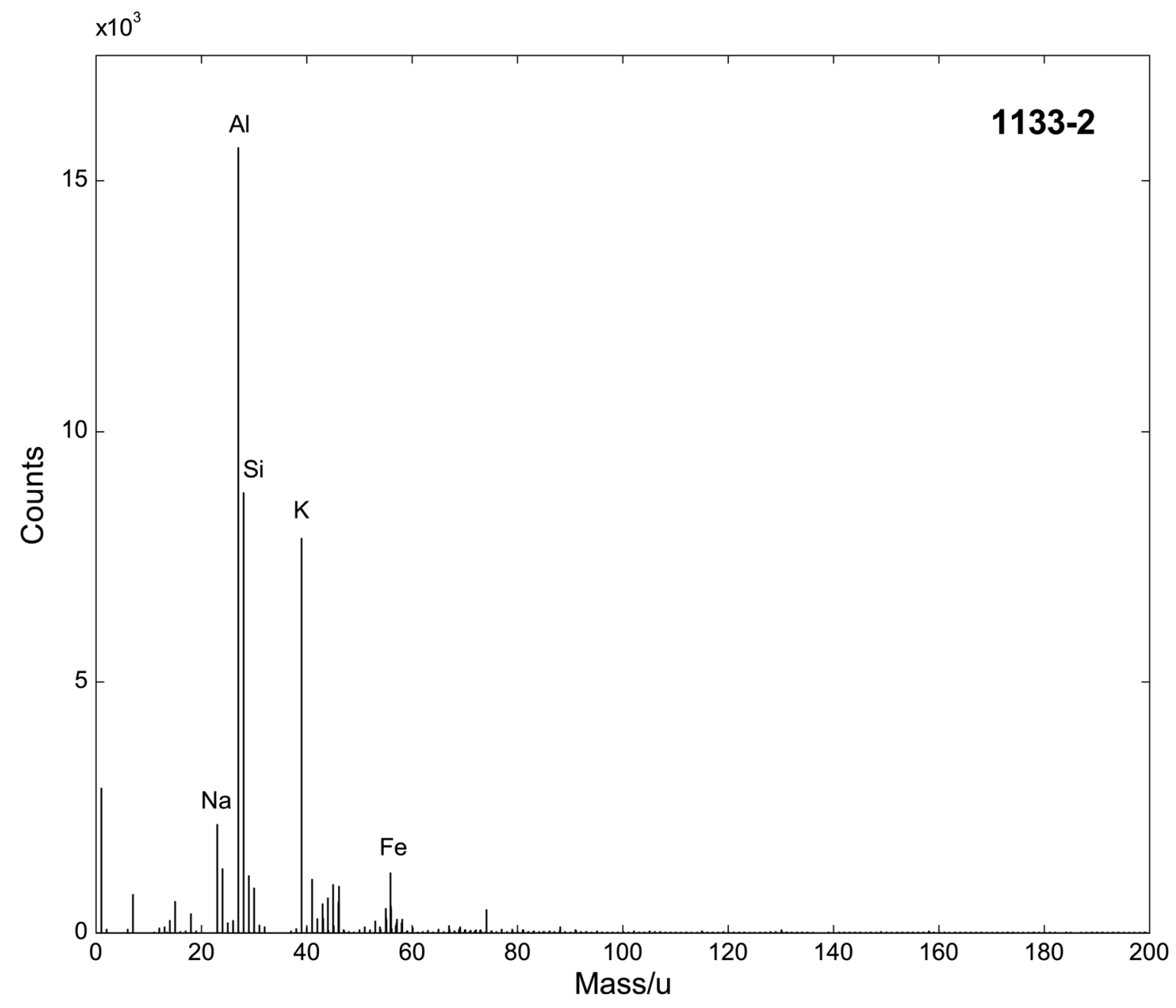

\begin{tabular}{rc}
\hline Accession \# & 01133-02 \\
\hline Host Material & Coal GAT034 \\
Technique & ToF-SIMS \\
Mass Range & 200 Da \\
Instrument & ION-TOF TOF-SIMS IV \\
Analyzer Type & time-of-flight, reflectron \\
Detector Type & $\sim 5000$ \\
Analyzer Mass Resolution & Electron multiplier \\
Specimen Normal to Analyzer & $0^{\circ}$ \\
Primary Beam lon Gun & ON-TOF \\
Primary Species & Ga \\
Primary lon Pulse Length & $25 \times 10^{-9}$ sec \\
Primary lon Pulse Rate & $10 \mathrm{kHz}$ \\
Net Beam Voltage & 25000 \\
Beam Current & $1.5 \mathrm{nA}$ \\
Beam Diameter & $0.1 \mu \mathrm{m}$ \\
Beam Raster Width & $500 \mu \mathrm{m} \times 500 \mu \mathrm{m}$ \\
Beam Incident Angle & $45^{\circ}\left(45^{\circ}\right.$ effective $)$ \\
Source to Analyzer Angle & $45^{\circ}$ \\
Comment & \\
\hline
\end{tabular}




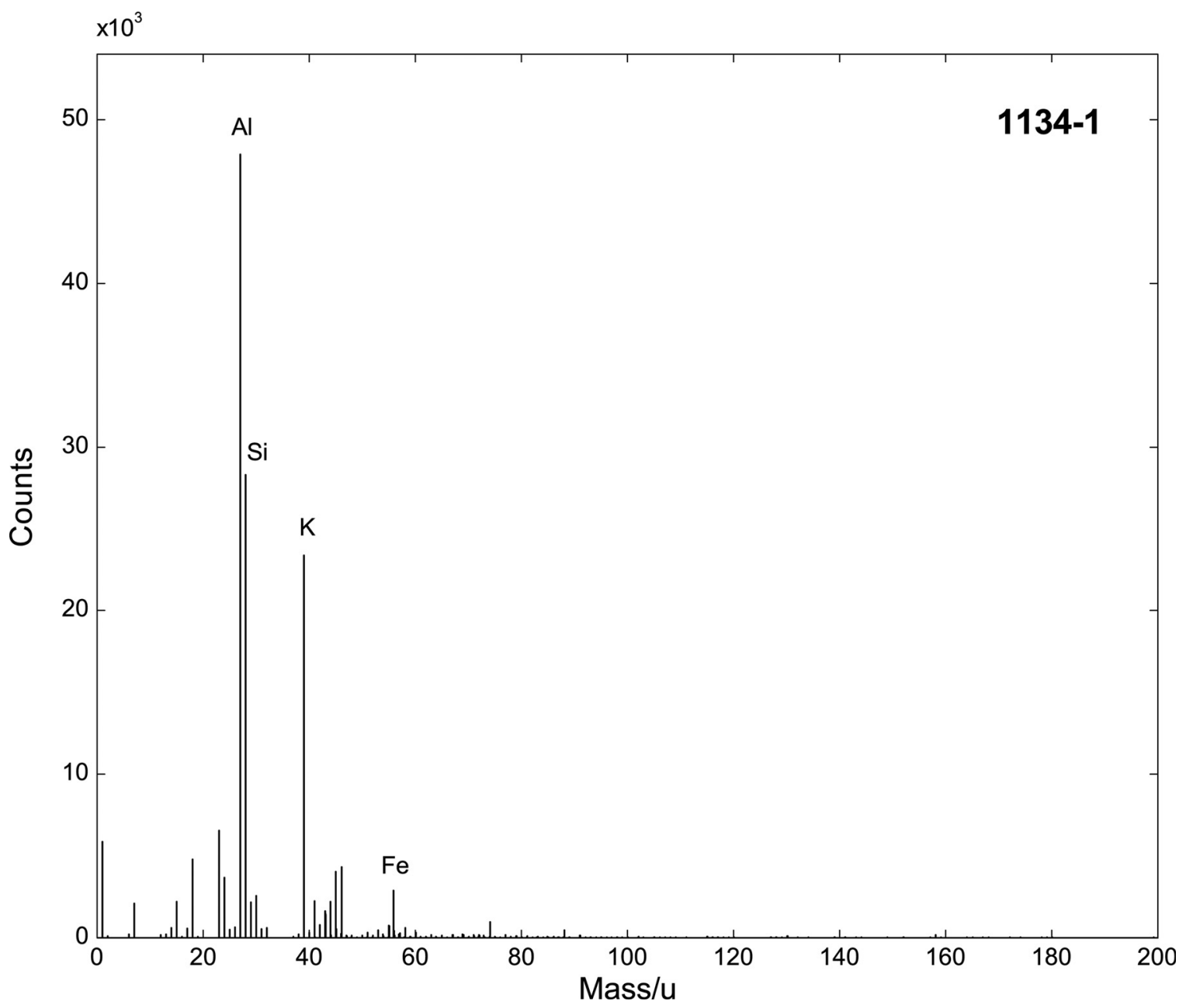

\begin{tabular}{rc}
\hline Accession \# & $\mathbf{0 1 1 3 4 - 0 1}$ \\
\hline Host Material & Coal GAT035 \\
Technique & ToF-SIMS \\
Mass Range & 200 Da \\
Instrument & ION-TOF TOF-SIMS IV \\
Analyzer Type & time-of-flight, reflectron \\
Detector Type & $\sim 5000$ \\
Analyzer Mass Resolution & Electron multiplier \\
Specimen Normal to Analyzer & $0^{\circ}$ \\
Primary Beam lon Gun & ION-TOF \\
Primary Species & $\mathrm{Ga}^{+}$ \\
Primary lon Pulse Length & $25 \times 10^{-9} \mathrm{sec}$ \\
Primary lon Pulse Rate & $10 \mathrm{kHz}$ \\
Net Beam Voltage & 25000 \\
Beam Current & $1.5 \mathrm{nA}$ \\
Beam Diameter & $0.1 \mu \mathrm{m}$ \\
Beam Raster Width & $500 \mu \mathrm{m} \times 500 \mu \mathrm{m}$ \\
Beam Incident Angle & $45^{\circ}\left(45^{\circ}\right.$ effective $)$ \\
Source to Analyzer Angle & $45^{\circ}$ \\
Comment & \\
\hline
\end{tabular}




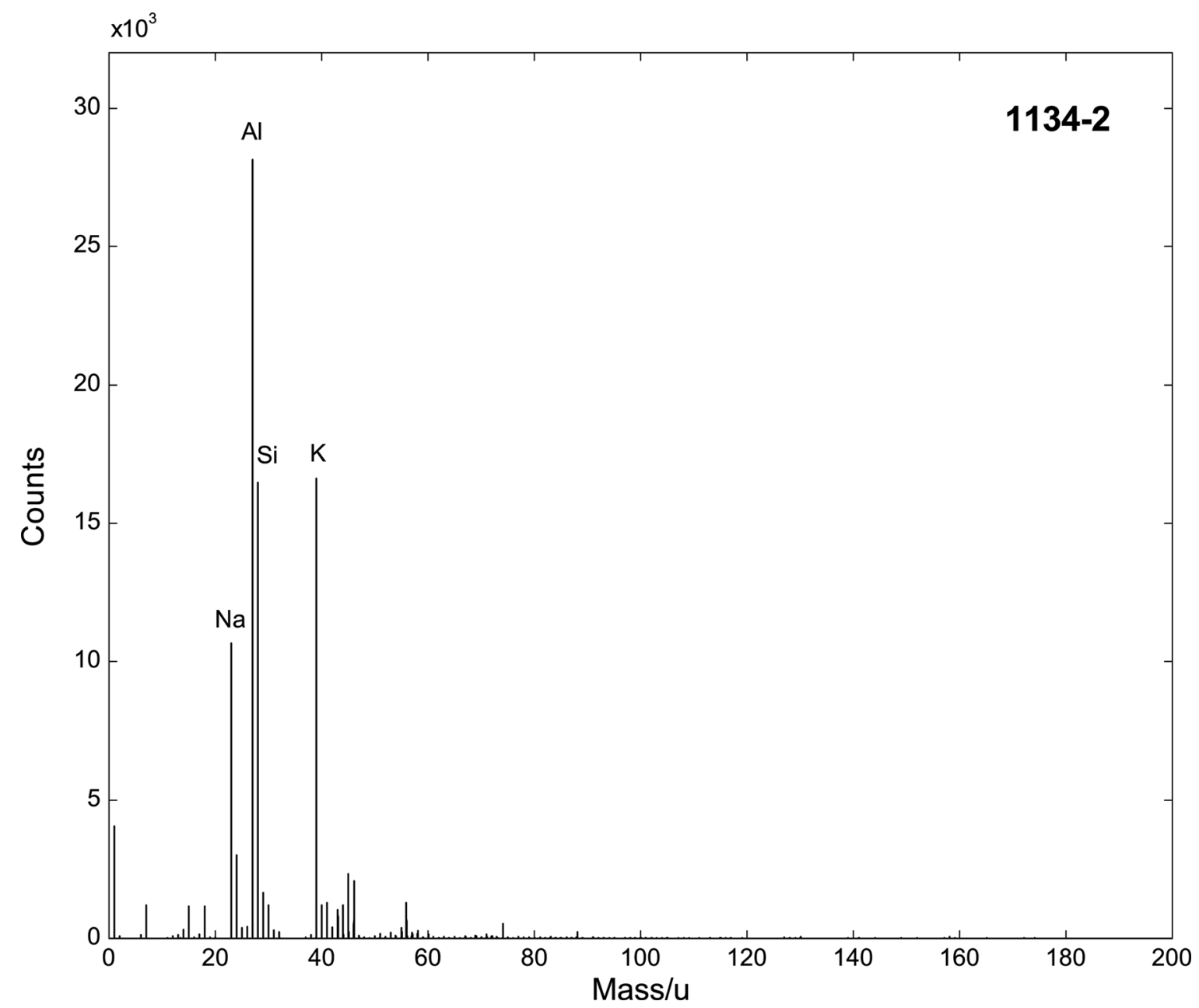

\begin{tabular}{rc}
\hline Accession \# & 01134-02 \\
\hline Host Material & Coal GAT035 \\
Technique & ToF-SIMS \\
Mass Range & 200 Da \\
Instrument & ION-TOF TOF-SIMS IV \\
Analyzer Type & time-of-flight, reflectron \\
Detector Type & $\sim 5000$ \\
Analyzer Mass Resolution & Electron multiplier \\
Specimen Normal to Analyzer & $0^{\circ}$ \\
Primary Beam lon Gun & ION-TOF \\
Primary Species & $\mathrm{Ga}^{+}$ \\
Primary lon Pulse Length & $25 \times 10^{-9} \mathrm{sec}$ \\
Primary lon Pulse Rate & $10 \mathrm{kHz}$ \\
Net Beam Voltage & 25000 \\
Beam Current & $1.5 \mathrm{nA}$ \\
Beam Diameter & $0.1 \mu \mathrm{m}$ \\
Beam Raster Width & $500 \mu \mathrm{m} \times 500 \mu \mathrm{m}$ \\
Beam Incident Angle & $45^{\circ}\left(45^{\circ}\right.$ effective $)$ \\
Source to Analyzer Angle & $45^{\circ}$ \\
Comment & \\
\hline
\end{tabular}




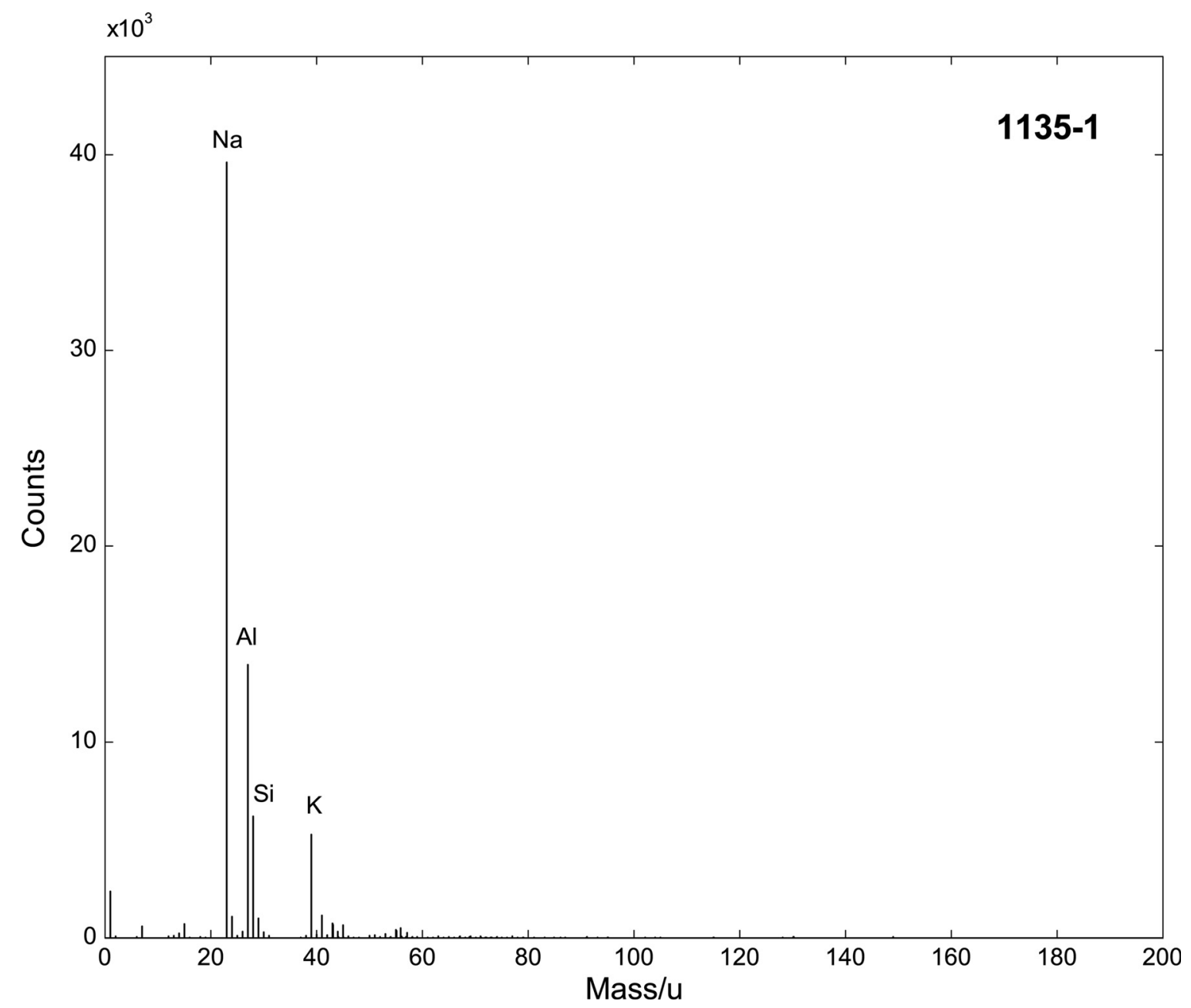

\begin{tabular}{rc}
\hline Accession \# & 01135-01 \\
\hline Host Material & Coal GBJ010 \\
Technique & ToF-SIMS \\
Mass Range & $200 \mathrm{Da}$ \\
Instrument & ION-TOF TOF-SIMS IV \\
Analyzer Type & time-of-flight, reflectron \\
Detector Type & $\sim 5000$ \\
Analyzer Mass Resolution & Electron multiplier \\
Specimen Normal to Analyzer & $0^{\circ}$ \\
Primary Beam lon Gun & ION-TOF \\
Primary Species & Ga \\
Primary lon Pulse Length & $25 \times 10^{-9}$ sec \\
Primary lon Pulse Rate & $10 \mathrm{kHz}$ \\
Net Beam Voltage & 25000 \\
Beam Current & $1.5 \mathrm{nA}$ \\
Beam Diameter & $0.1 \mu \mathrm{m}$ \\
Beam Raster Width & $500 \mu \mathrm{m} \times 500 \mu \mathrm{m}$ \\
Beam Incident Angle & $45^{\circ}\left(45^{\circ}\right.$ effective $)$ \\
Source to Analyzer Angle & $45^{\circ}$ \\
Comment & \\
\hline
\end{tabular}




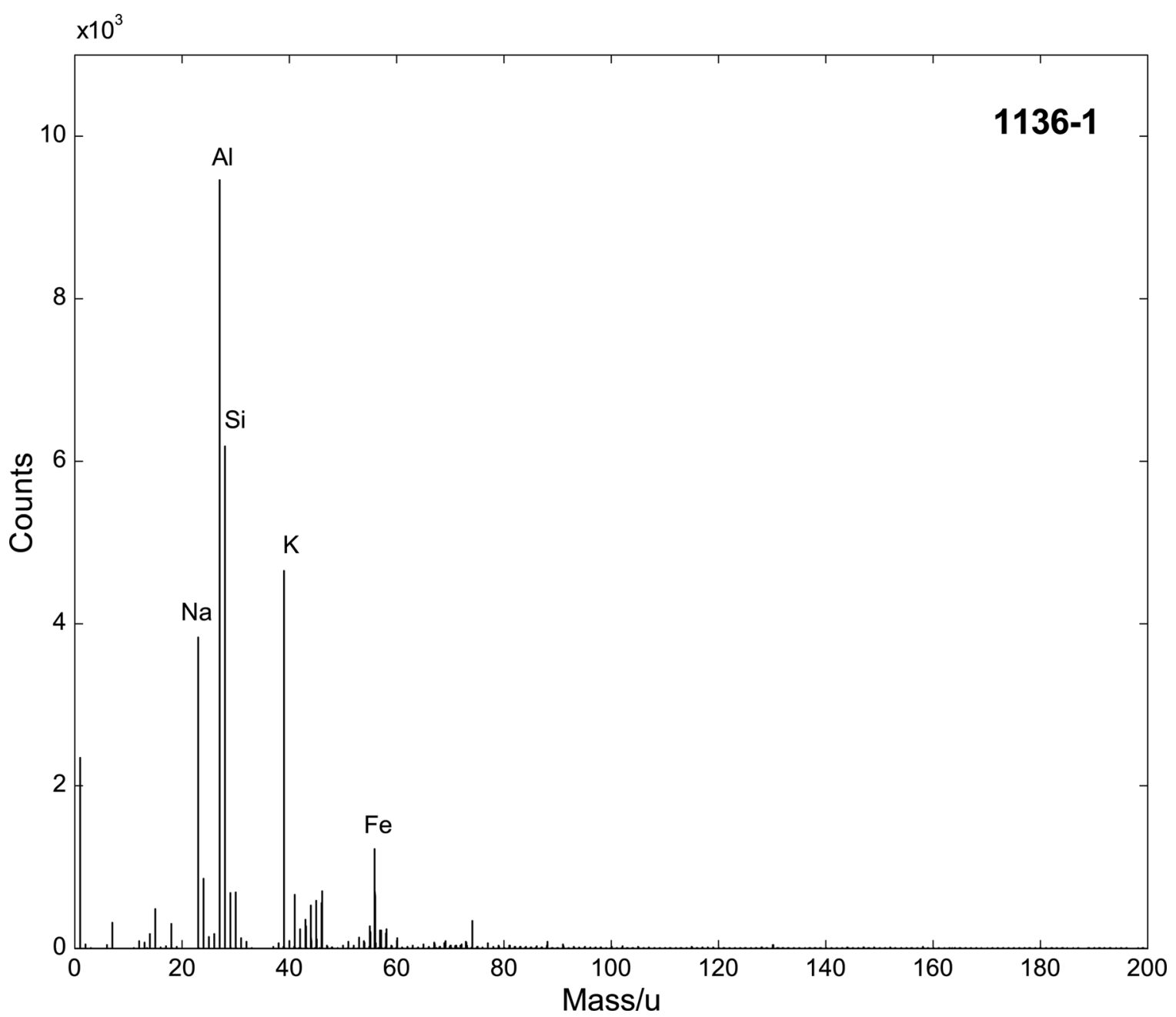

\begin{tabular}{rc}
\hline Accession \# & 01136-01 \\
\hline Host Material & Coal GLJ005 \\
Technique & ToF-SIMS \\
Mass Range & 200 Da \\
Instrument & ION-TOF TOF-SIMS IV \\
Analyzer Type & time-of-flight, reflectron \\
Detector Type & $\sim 5000$ \\
Analyzer Mass Resolution & Electron multiplier \\
Specimen Normal to Analyzer & $0^{\circ}$ \\
Primary Beam lon Gun & ION-TOF \\
Primary Species & Ga \\
Primary lon Pulse Length & $25 \times 10^{-9}$ sec \\
Primary lon Pulse Rate & $10 \mathrm{kHz}$ \\
Net Beam Voltage & 25000 \\
Beam Current & $1.5 \mathrm{nA}$ \\
Beam Diameter & $0.1 \mu \mathrm{m}$ \\
Beam Raster Width & $500 \mu \mathrm{m} \times 500 \mu \mathrm{m}$ \\
Beam Incident Angle & $45^{\circ}\left(45^{\circ}\right.$ effective $)$ \\
Source to Analyzer Angle & $45^{\circ}$ \\
Comment & \\
\hline
\end{tabular}




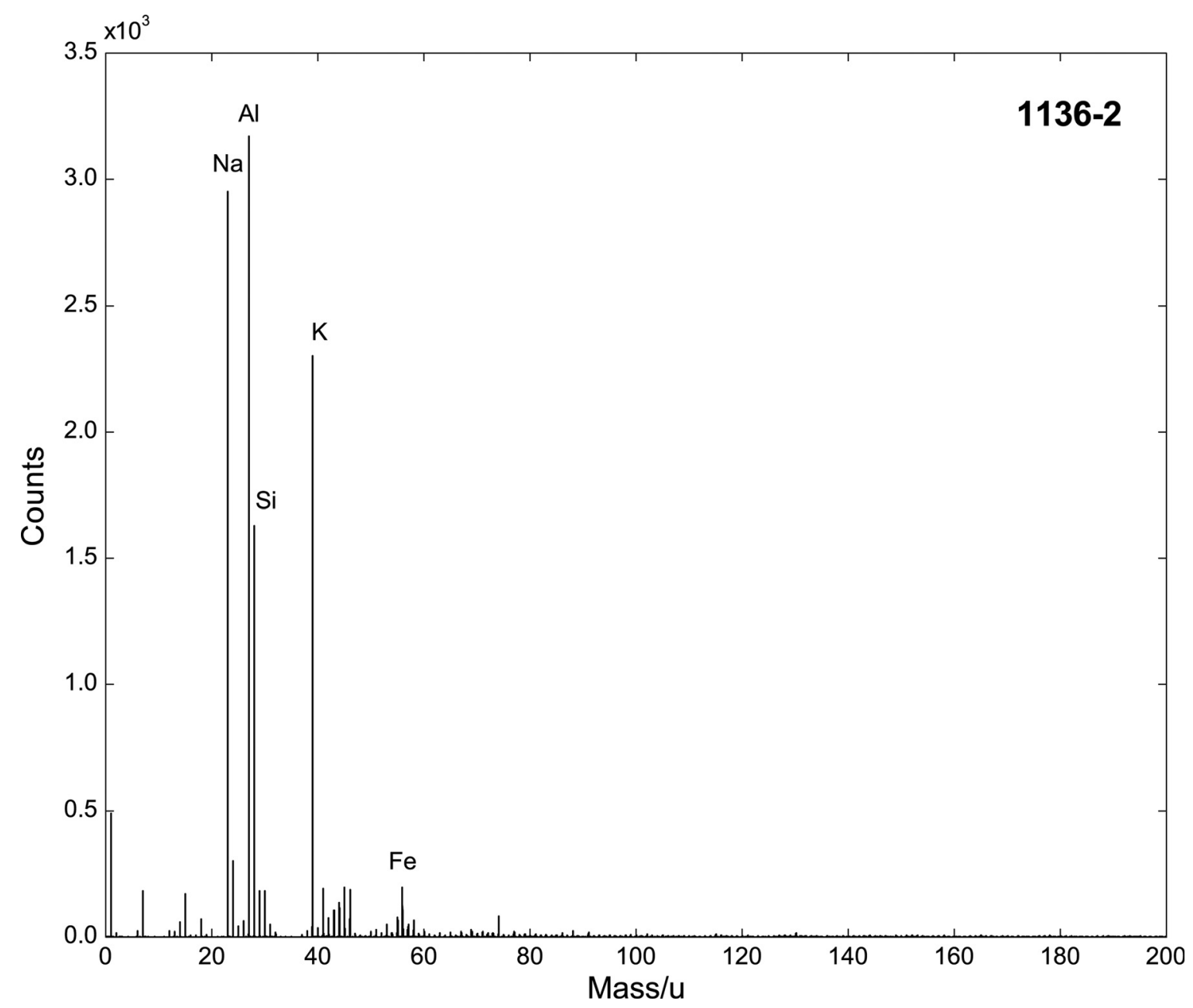

\begin{tabular}{rc}
\hline Accession \# & 01136-02 \\
\hline Host Material & Coal GLJ005 \\
Technique & ToF-SIMS \\
Mass Range & 200 Da \\
Instrument & ION-TOF TOF-SIMS IV \\
Analyzer Type & time-of-flight, reflectron \\
Detector Type & $\sim 5000$ \\
Analyzer Mass Resolution & Electron multiplier \\
Specimen Normal to Analyzer & $0^{\circ}$ \\
Primary Beam lon Gun & ION-TOF \\
Primary Species & Ga ${ }^{+}$ \\
Primary lon Pulse Length & $25 \times 10^{-9}$ sec \\
Primary lon Pulse Rate & $10 \mathrm{kHz}$ \\
Net Beam Voltage & 25000 \\
Beam Current & $1.5 \mathrm{nA}$ \\
Beam Diameter & $0.1 \mu \mathrm{m}$ \\
Beam Raster Width & $500 \mu \mathrm{m} \times 500 \mu \mathrm{m}$ \\
Beam Incident Angle & $45^{\circ}\left(45^{\circ}\right.$ effective $)$ \\
Source to Analyzer Angle & $45^{\circ}$ \\
Comment & \\
\hline
\end{tabular}




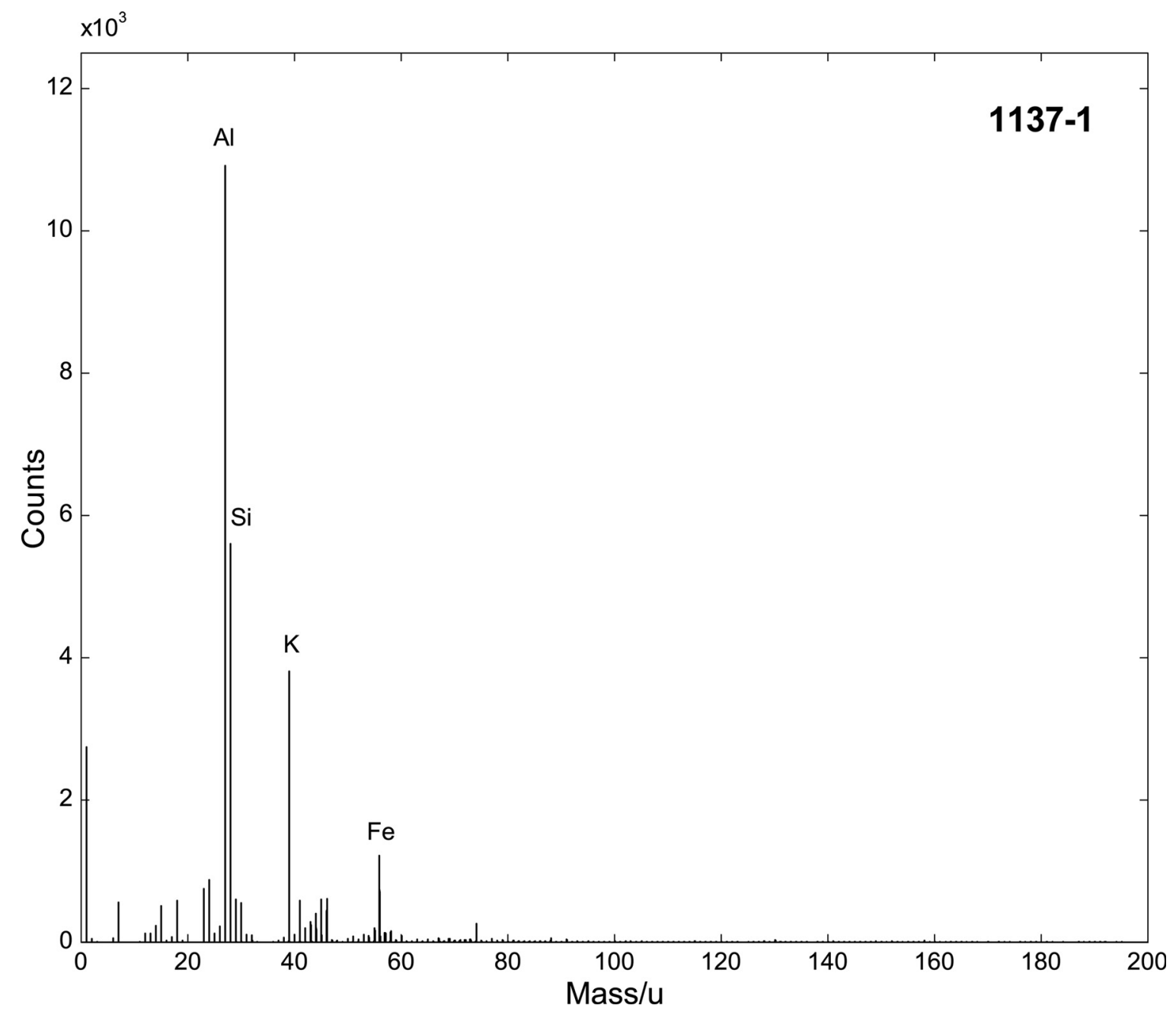

\begin{tabular}{|c|c|}
\hline Accession \# & 01137-01 \\
\hline Host Material & Coal GPB062 \\
\hline Technique & ToF-SIMS \\
\hline Mass Range & $200 \mathrm{Da}$ \\
\hline Instrument & ION-TOF TOF-SIMS IV \\
\hline Analyzer Type & time-of-flight, reflectron \\
\hline Analyzer Mass Resolution & $\sim 5000$ \\
\hline Detector Type & Electron multiplier \\
\hline Specimen Normal to Analyzer & $0^{\circ}$ \\
\hline Primary Beam Ion Gun & ION-TOF \\
\hline Primary Species & $\mathrm{Ga}^{+}$ \\
\hline Primary Ion Pulse Length & $25 \times 10^{-9} \mathrm{sec}$ \\
\hline Primary Ion Pulse Rate & $10 \mathrm{kHz}$ \\
\hline Net Beam Voltage & 25000 \\
\hline Beam Current & $1.5 \mathrm{nA}$ \\
\hline Beam Diameter & $0.1 \mu \mathrm{m}$ \\
\hline Beam Raster Width & $500 \mu \mathrm{m} \times 500 \mu \mathrm{m}$ \\
\hline Beam Incident Angle & $45^{\circ}\left(45^{\circ}\right.$ effective $)$ \\
\hline Source to Analyzer Angle & $45^{\circ}$ \\
\hline Comment & \\
\hline
\end{tabular}




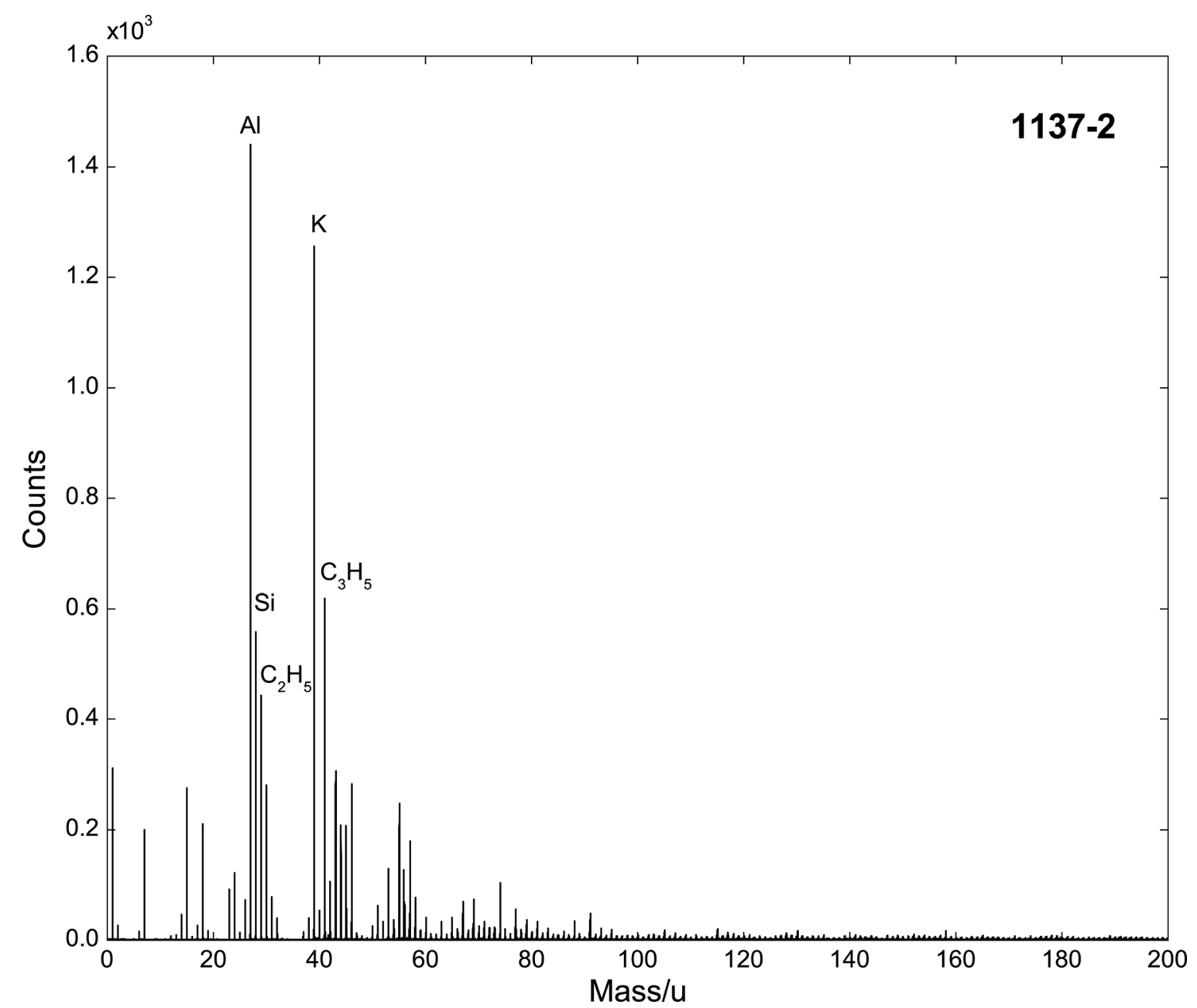

\begin{tabular}{rc}
\hline Accession \# & 01137-02 \\
\hline Host Material & Coal GPB062 \\
Technique & ToF-SIMS \\
Mass Range & $200 \mathrm{Da}$ \\
Instrument & ION-TOF TOF-SIMS IV \\
Analyzer Type & time-of-flight, reflectron \\
Detector Type & $\sim 5000$ \\
Analyzer Mass Resolution & Electron multiplier \\
Specimen Normal to Analyzer & $0^{\circ}$ \\
Primary Beam lon Gun & ION-TOF \\
Primary Species & Ga \\
Primary lon Pulse Length & $25 \times 10^{-9}$ sec \\
Primary lon Pulse Rate & $10 \mathrm{kHz}$ \\
Net Beam Voltage & 25000 \\
Beam Current & $1.5 \mathrm{nA}$ \\
Beam Diameter & $0.1 \mu \mathrm{m}$ \\
Beam Raster Width & $500 \mu \mathrm{m} \times 500 \mu \mathrm{m}$ \\
Beam Incident Angle & $45^{\circ}\left(45^{\circ}\right.$ effective $)$ \\
Source to Analyzer Angle & $45^{\circ}$ \\
Comment & \\
\hline
\end{tabular}




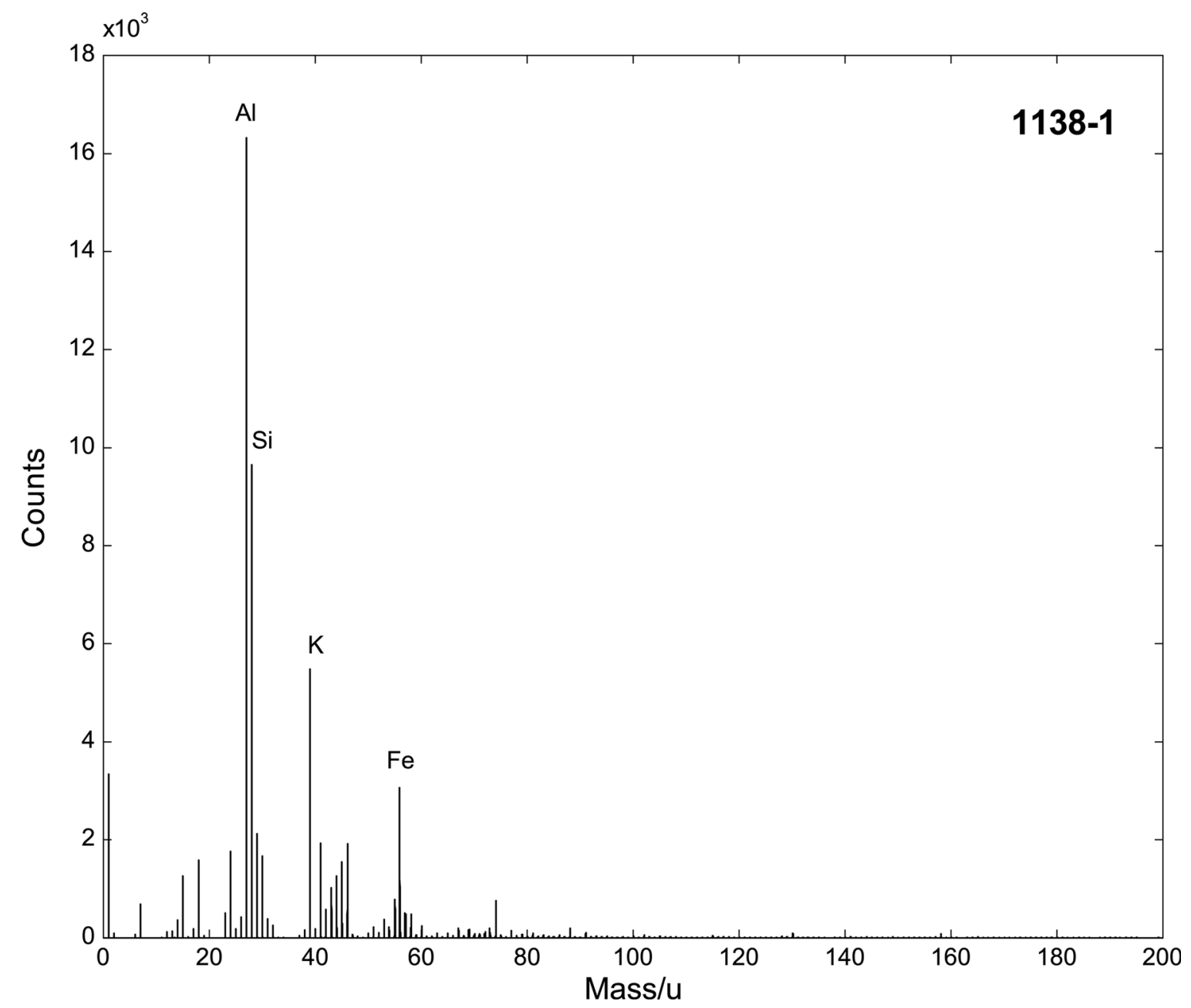

\begin{tabular}{rc}
\hline Accession \# & $\mathbf{0 1 1 3 8 - 0 1}$ \\
\hline Host Material & Coal HGB011 \\
Technique & ToF-SIMS \\
Mass Range & $200 \mathrm{Da}$ \\
Instrument & ION-TOF TOF-SIMS IV \\
Analyzer Type & time-of-flight, reflectron \\
Detector Type & $\sim 5000$ \\
Analyzer Mass Resolution & Electron multiplier \\
Specimen Normal to Analyzer & $0^{\circ}$ \\
Primary Beam lon Gun & ION-TOF \\
Primary Species & $\mathrm{Ga}^{+}$ \\
Primary lon Pulse Length & $25 \times 10^{-9} \mathrm{sec}$ \\
Primary lon Pulse Rate & $10 \mathrm{kHz}$ \\
Net Beam Voltage & 25000 \\
Beam Current & $1.5 \mathrm{nA}$ \\
Beam Diameter & $0.1 \mu \mathrm{m}$ \\
Beam Raster Width & $500 \mu \mathrm{m} \times 500 \mu \mathrm{m}$ \\
Beam Incident Angle & $45^{\circ}\left(45^{\circ}\right.$ effective $)$ \\
Source to Analyzer Angle & $45^{\circ}$ \\
Comment & \\
\hline
\end{tabular}




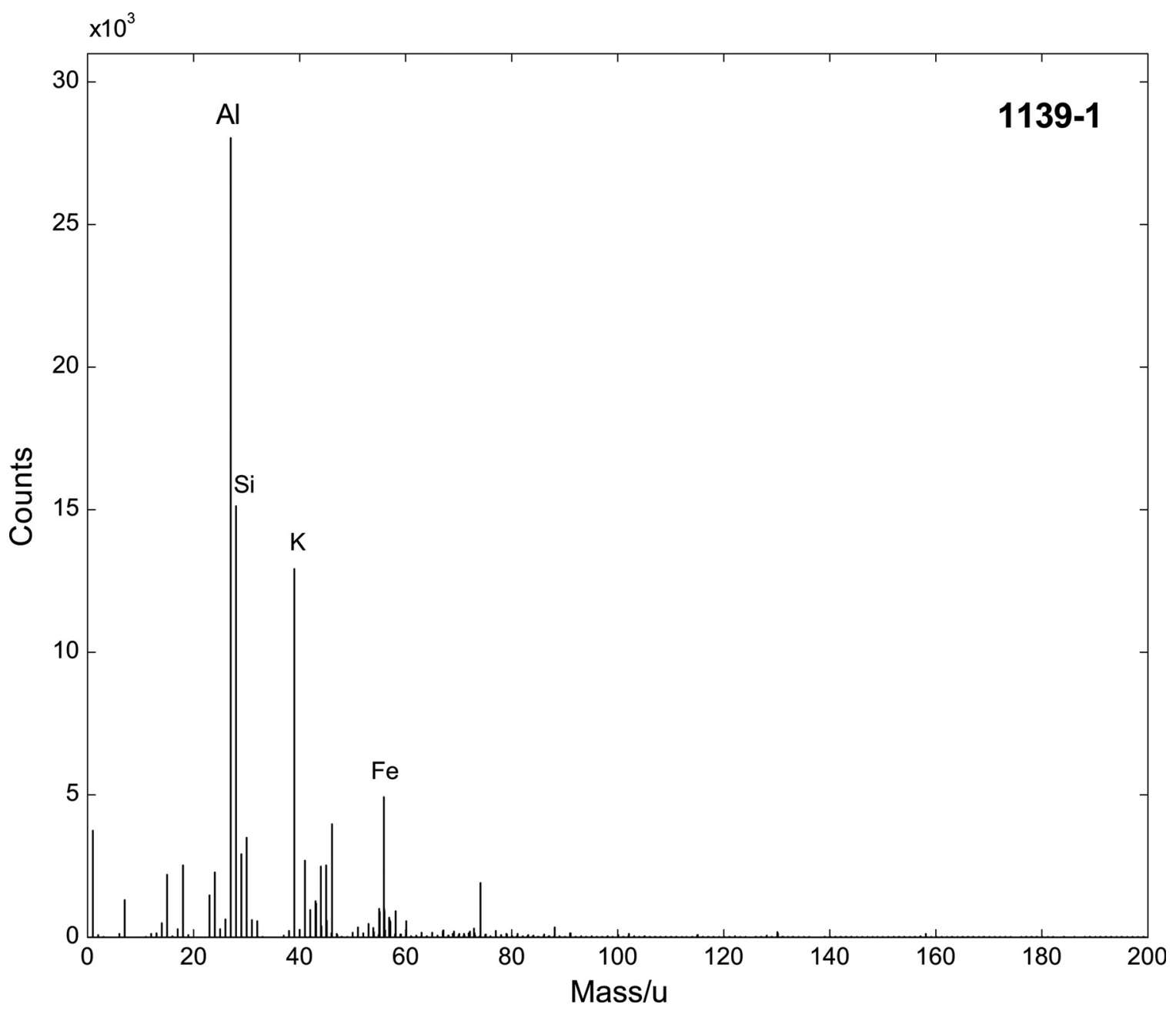

\begin{tabular}{|c|c|}
\hline Accession \# & 01139-01 \\
\hline Host Material & Coal HNA015 \\
\hline Technique & ToF-SIMS \\
\hline Mass Range & $200 \mathrm{Da}$ \\
\hline Instrument & ION-TOF TOF-SIMS IV \\
\hline Analyzer Type & time-of-flight, reflectron \\
\hline Analyzer Mass Resolution & $\sim 5000$ \\
\hline Detector Type & Electron multiplier \\
\hline Specimen Normal to Analyzer & $0^{\circ}$ \\
\hline Primary Beam Ion Gun & ION-TOF \\
\hline Primary Species & $\mathrm{Ga}^{+}$ \\
\hline Primary Ion Pulse Length & $25 \times 10^{-9} \mathrm{sec}$ \\
\hline Primary Ion Pulse Rate & $10 \mathrm{kHz}$ \\
\hline Net Beam Voltage & 25000 \\
\hline Beam Current & $1.5 \mathrm{nA}$ \\
\hline Beam Diameter & $0.1 \mu \mathrm{m}$ \\
\hline Beam Raster Width & $500 \mu \mathrm{m} \times 500 \mu \mathrm{m}$ \\
\hline Beam Incident Angle & $45^{\circ}\left(45^{\circ}\right.$ effective $)$ \\
\hline Source to Analyzer Angle & $45^{\circ}$ \\
\hline Comment & \\
\hline
\end{tabular}




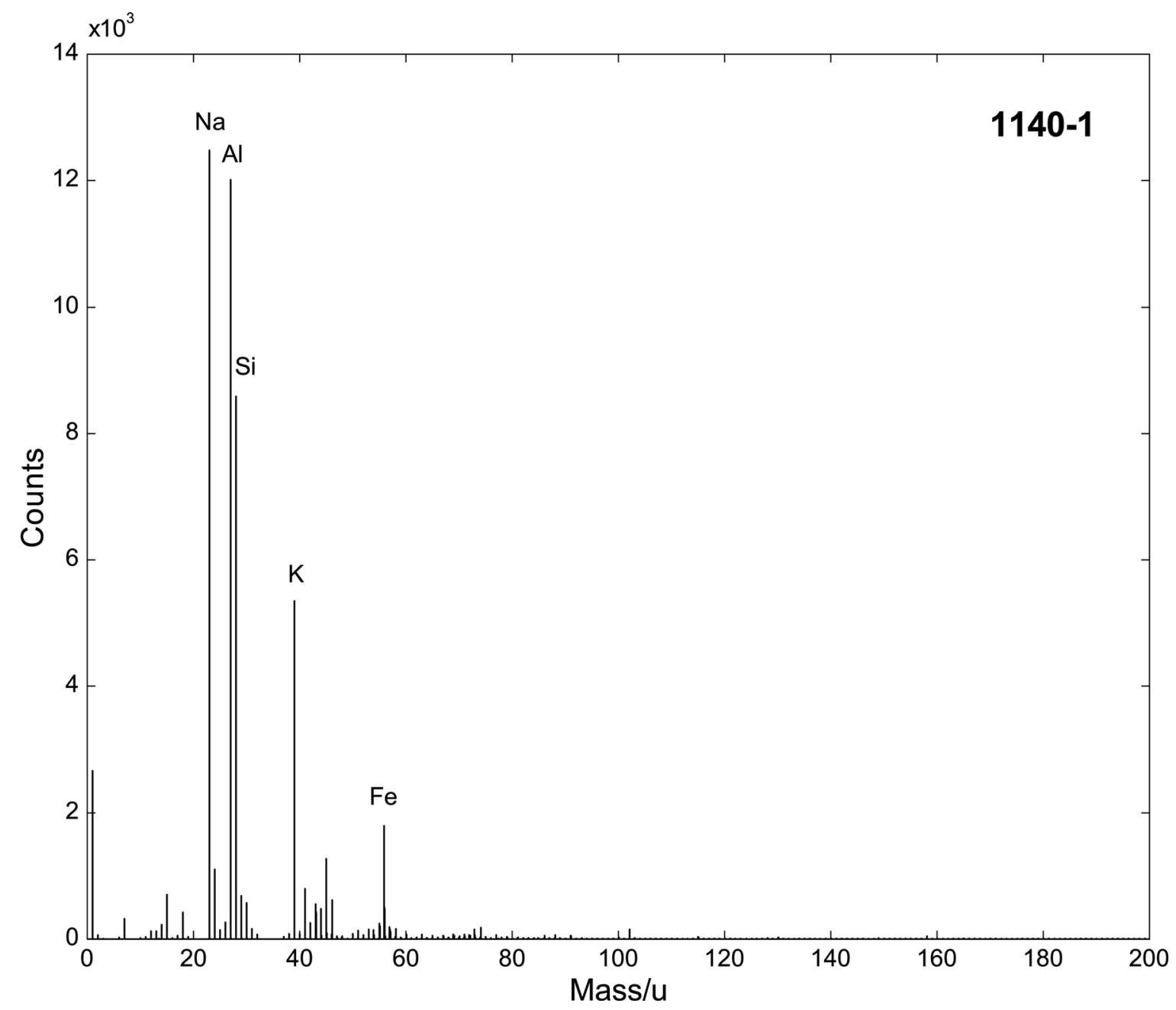

\begin{tabular}{rc}
\hline Accession \# & $\mathbf{0 1 1 4 0 - 0 1}$ \\
\hline Host Material & Coal ILL6 \\
Technique & ToF-SIMS \\
Mass Range & $200 \mathrm{Da}$ \\
Instrument & ION-TOF TOF-SIMS IV \\
Analyzer Type & time-of-flight, reflectron \\
Detector Type & $\sim 5000$ \\
Analyzer Mass Resolution & Electron multiplier \\
Specimen Normal to Analyzer & $0^{\circ}$ \\
Primary Beam lon Gun & ION-TOF \\
Primary Species & Ga \\
Primary lon Pulse Length & $25 \times 10^{-9} \mathrm{sec}$ \\
Primary lon Pulse Rate & $10 \mathrm{kHz}$ \\
Net Beam Voltage & 25000 \\
Beam Current & $1.5 \mathrm{nA}$ \\
Beam Diameter & $0.1 \mu \mathrm{m}$ \\
Beam Raster Width & $500 \mu \mathrm{m} \times 500 \mu \mathrm{m}$ \\
Beam Incident Angle & $45^{\circ}\left(45^{\circ}\right.$ effective $)$ \\
Source to Analyzer Angle & $45^{\circ}$ \\
Comment & \\
\hline
\end{tabular}




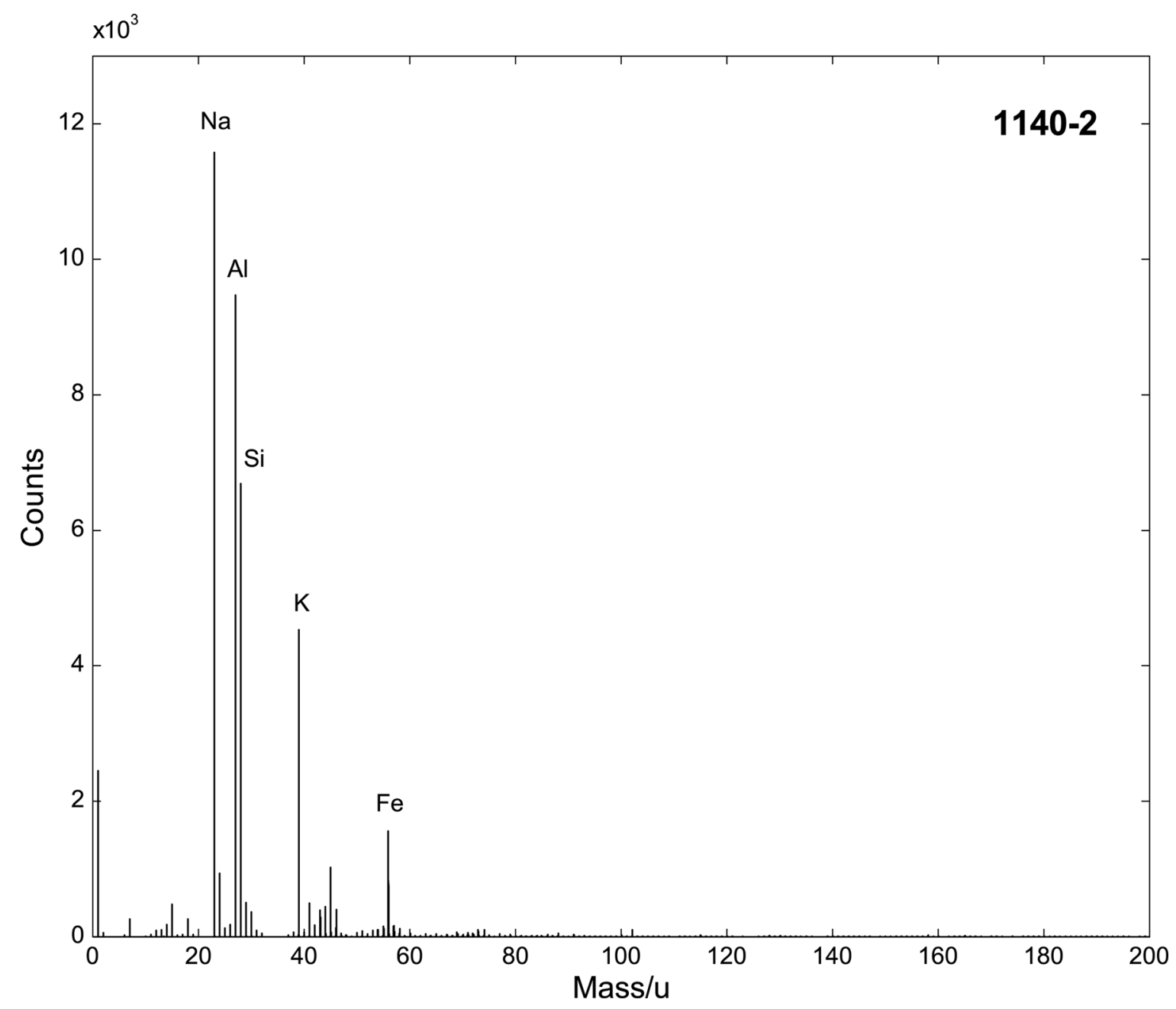

\begin{tabular}{|c|c|}
\hline Accession \# & 01140-02 \\
\hline Host Material & Coal ILL6 \\
\hline Technique & ToF-SIMS \\
\hline Mass Range & $200 \mathrm{Da}$ \\
\hline Instrument & ION-TOF TOF-SIMS IV \\
\hline Analyzer Type & time-of-flight, reflectron \\
\hline Analyzer Mass Resolution & $\sim 5000$ \\
\hline Detector Type & Electron multiplier \\
\hline Specimen Normal to Analyzer & $0^{\circ}$ \\
\hline Primary Beam Ion Gun & ION-TOF \\
\hline Primary Species & $\mathrm{Ga}^{+}$ \\
\hline Primary Ion Pulse Length & $25 \times 10^{-9} \mathrm{sec}$ \\
\hline Primary Ion Pulse Rate & $10 \mathrm{kHz}$ \\
\hline Net Beam Voltage & 25000 \\
\hline Beam Current & $1.5 \mathrm{nA}$ \\
\hline Beam Diameter & $0.1 \mu \mathrm{m}$ \\
\hline Beam Raster Width & $500 \mu \mathrm{m} \times 500 \mu \mathrm{m}$ \\
\hline Beam Incident Angle & $45^{\circ}\left(45^{\circ}\right.$ effective $)$ \\
\hline Source to Analyzer Angle & $45^{\circ}$ \\
\hline Comment & \\
\hline
\end{tabular}




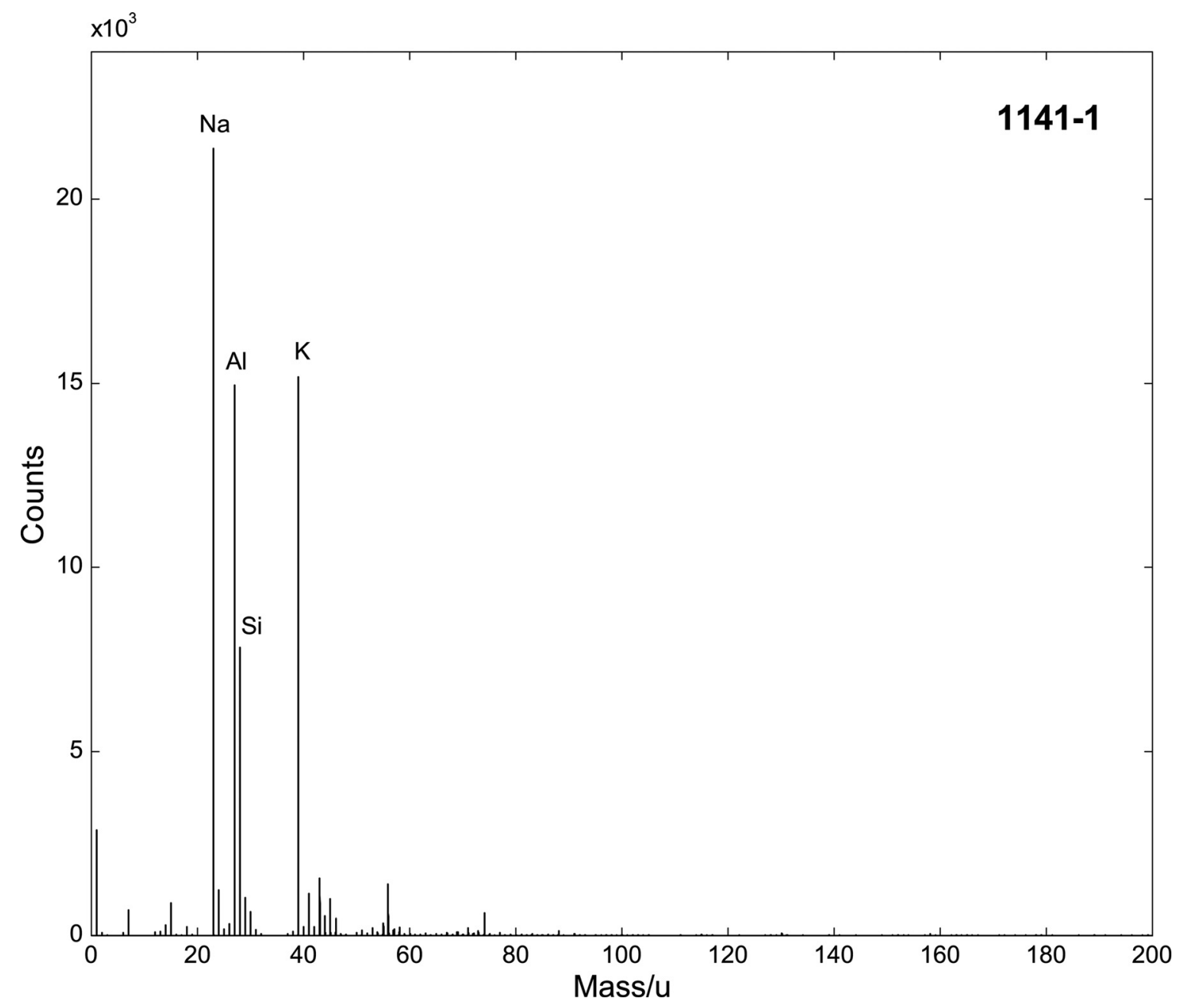

\begin{tabular}{rc}
\hline Accession \# & 01141-01 \\
\hline Host Material & Coal JIG014 \\
Technique & ToF-SIMS \\
Mass Range & $200 \mathrm{Da}$ \\
Instrument & ION-TOF TOF-SIMS IV \\
Analyzer Type & time-of-flight, reflectron \\
Detector Type & $\sim 5000$ \\
Analyzer Mass Resolution & Electron multiplier \\
Specimen Normal to Analyzer & $0^{\circ}$ \\
Primary Beam lon Gun & ON-TOF \\
Primary Species & Ga \\
Primary lon Pulse Length & $25 \times 10^{-9} \mathrm{sec}$ \\
Primary lon Pulse Rate & $10 \mathrm{kHz}$ \\
Net Beam Voltage & 25000 \\
Beam Current & $1.5 \mathrm{nA}$ \\
Beam Diameter & $0.1 \mu \mathrm{m}$ \\
Beam Raster Width & $500 \mu \mathrm{m} \times 500 \mu \mathrm{m}$ \\
Beam Incident Angle & $45^{\circ}\left(45^{\circ}\right.$ effective $)$ \\
Source to Analyzer Angle & $45^{\circ}$ \\
Comment & \\
\hline
\end{tabular}




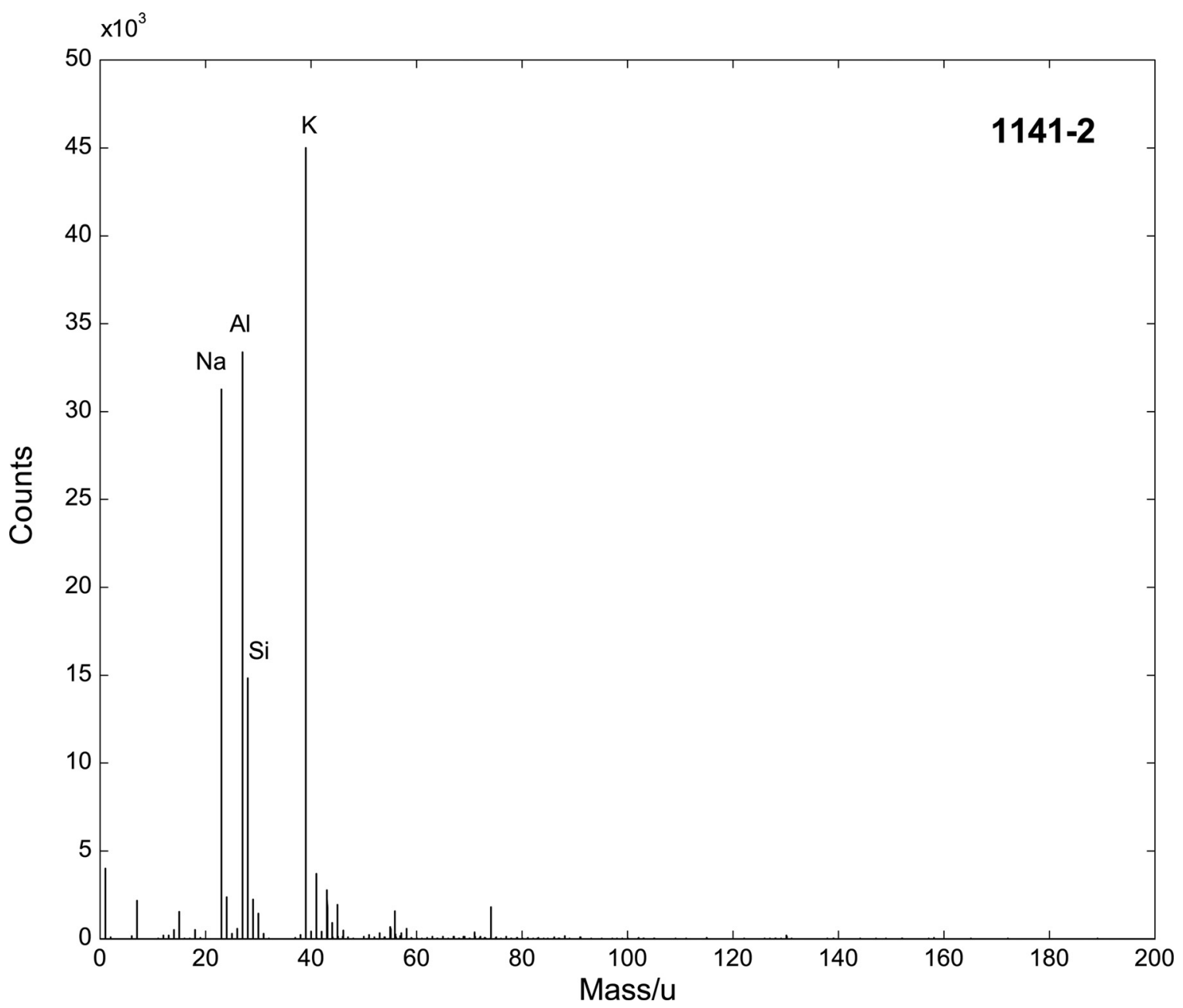

\begin{tabular}{rc}
\hline Accession \# & 01141-02 \\
\hline Host Material & Coal JIG014 \\
Technique & ToF-SIMS \\
Mass Range & $200 \mathrm{Da}$ \\
Instrument & ION-TOF TOF-SIMS IV \\
Analyzer Type & time-of-flight, reflectron \\
Detector Type & $\sim 5000$ \\
Analyzer Mass Resolution & Electron multiplier \\
Specimen Normal to Analyzer & $0^{\circ}$ \\
Primary Beam lon Gun & ION-TOF \\
Primary Species & Ga \\
Primary lon Pulse Length & $25 \times 10^{-9}$ sec \\
Primary lon Pulse Rate & $10 \mathrm{kHz}$ \\
Net Beam Voltage & 25000 \\
Beam Current & $1.5 \mathrm{nA}$ \\
Beam Diameter & $0.1 \mu \mathrm{m}$ \\
Beam Raster Width & $500 \mu \mathrm{m} \times 500 \mu \mathrm{m}$ \\
Beam Incident Angle & $45^{\circ}\left(45^{\circ}\right.$ effective $)$ \\
Source to Analyzer Angle & $45^{\circ}$ \\
Comment & \\
\hline
\end{tabular}




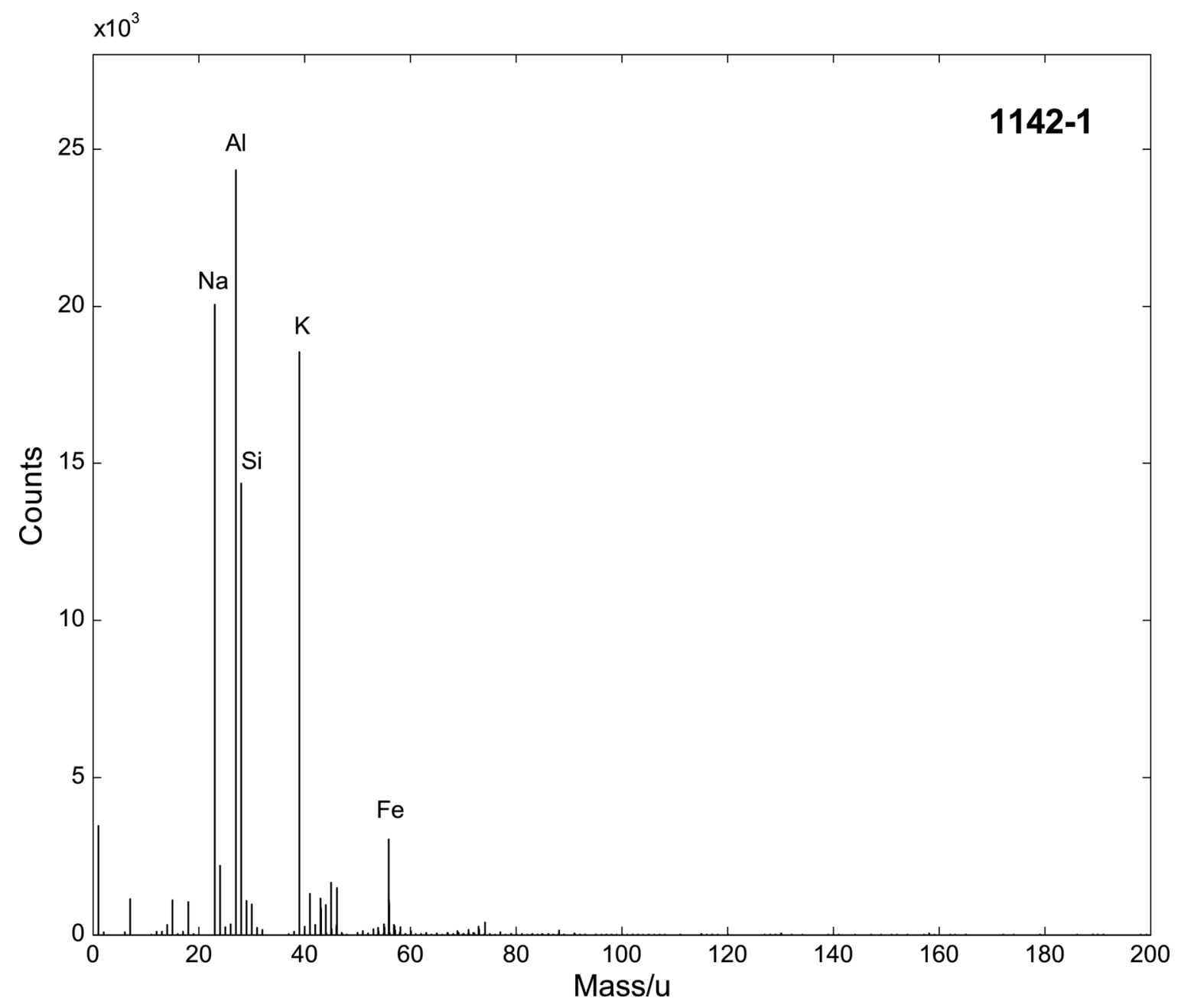

\begin{tabular}{rc}
\hline Accession \# & 01142-01 \\
\hline Host Material & Coal JIG017 \\
Technique & ToF-SIMS \\
Mass Range & 200 Da \\
Instrument & ION-TOF TOF-SIMS IV \\
Analyzer Type & time-of-flight, reflectron \\
Detector Type & $\sim 5000$ \\
Analyzer Mass Resolution & Electron multiplier \\
Specimen Normal to Analyzer & $0^{\circ}$ \\
Primary Beam lon Gun & ION-TOF \\
Primary Species & Ga \\
Primary lon Pulse Length & $25 \times 10^{-9} \mathrm{sec}$ \\
Primary lon Pulse Rate & $10 \mathrm{kHz}$ \\
Net Beam Voltage & 25000 \\
Beam Current & $1.5 \mathrm{nA}$ \\
Beam Diameter & $0.1 \mu \mathrm{m}$ \\
Beam Raster Width & $500 \mu \mathrm{m} \times 500 \mu \mathrm{m}$ \\
Beam Incident Angle & $45^{\circ}\left(45^{\circ}\right.$ effective $)$ \\
Source to Analyzer Angle & $45^{\circ}$ \\
Comment & \\
\hline
\end{tabular}




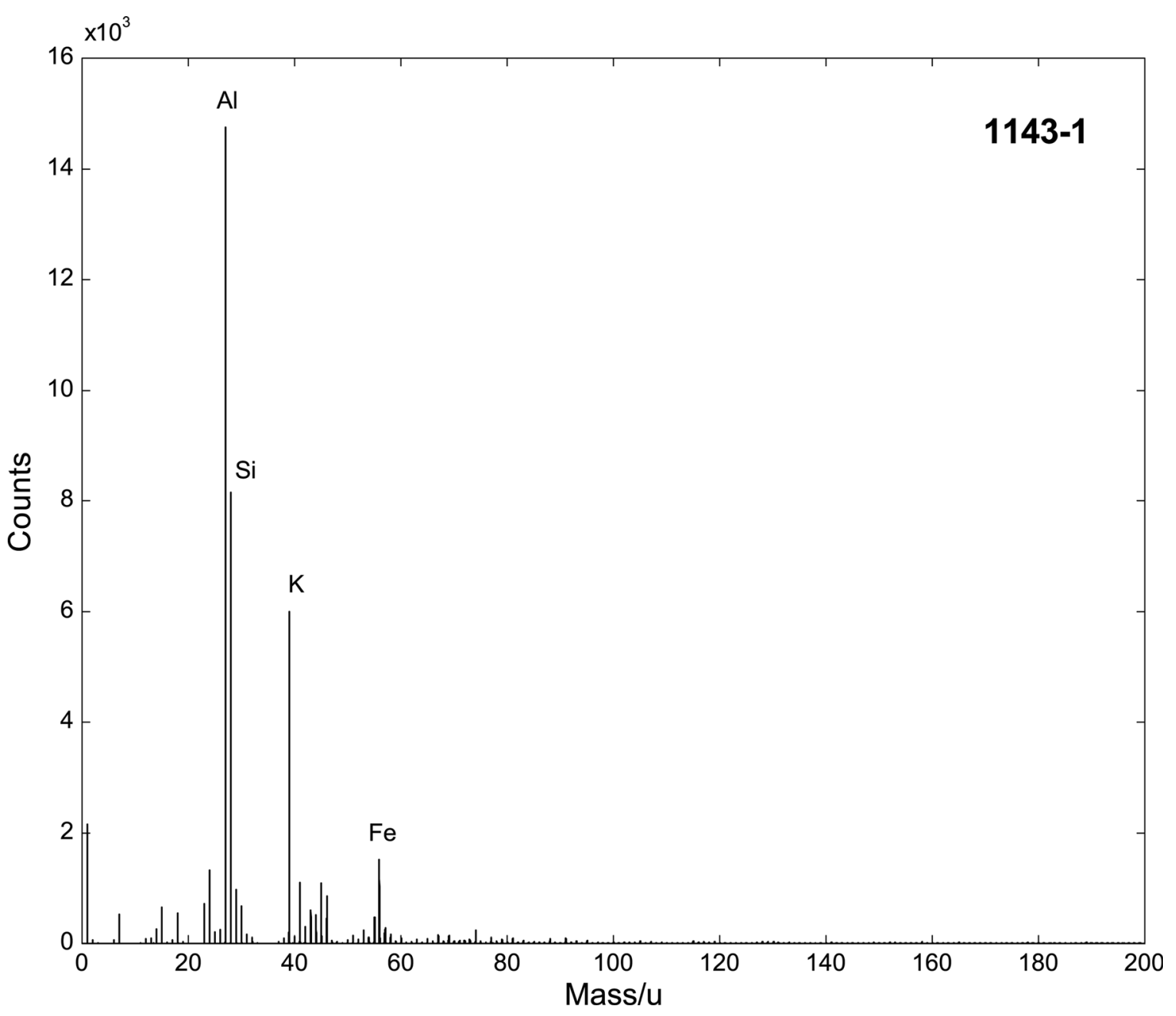

\begin{tabular}{rc}
\hline Accession \# & 01143-01 \\
\hline Host Material & Coal PCT010 \\
Technique & ToF-SIMS \\
Mass Range & $200 \mathrm{Da}$ \\
Instrument & ION-TOF TOF-SIMS IV \\
Analyzer Type & time-of-flight, reflectron \\
Detector Type & $\sim 5000$ \\
Analyzer Mass Resolution & Electron multiplier \\
Specimen Normal to Analyzer & $0^{\circ}$ \\
Primary Beam Ion Gun & ION-TOF \\
Primary Species & Ga ${ }^{+}$ \\
Primary lon Pulse Length & $25 \times 10^{-9}$ sec \\
Primary lon Pulse Rate & $10 \mathrm{kHz}$ \\
Net Beam Voltage & 25000 \\
Beam Current & $1.5 \mathrm{nA}$ \\
Beam Diameter & $0.1 \mu \mathrm{m}$ \\
Beam Raster Width & $500 \mu \mathrm{m} \times 500 \mu \mathrm{m}$ \\
Beam Incident Angle & $45^{\circ}\left(45^{\circ}\right.$ effective $)$ \\
Source to Analyzer Angle & $45^{\circ}$ \\
\hline
\end{tabular}




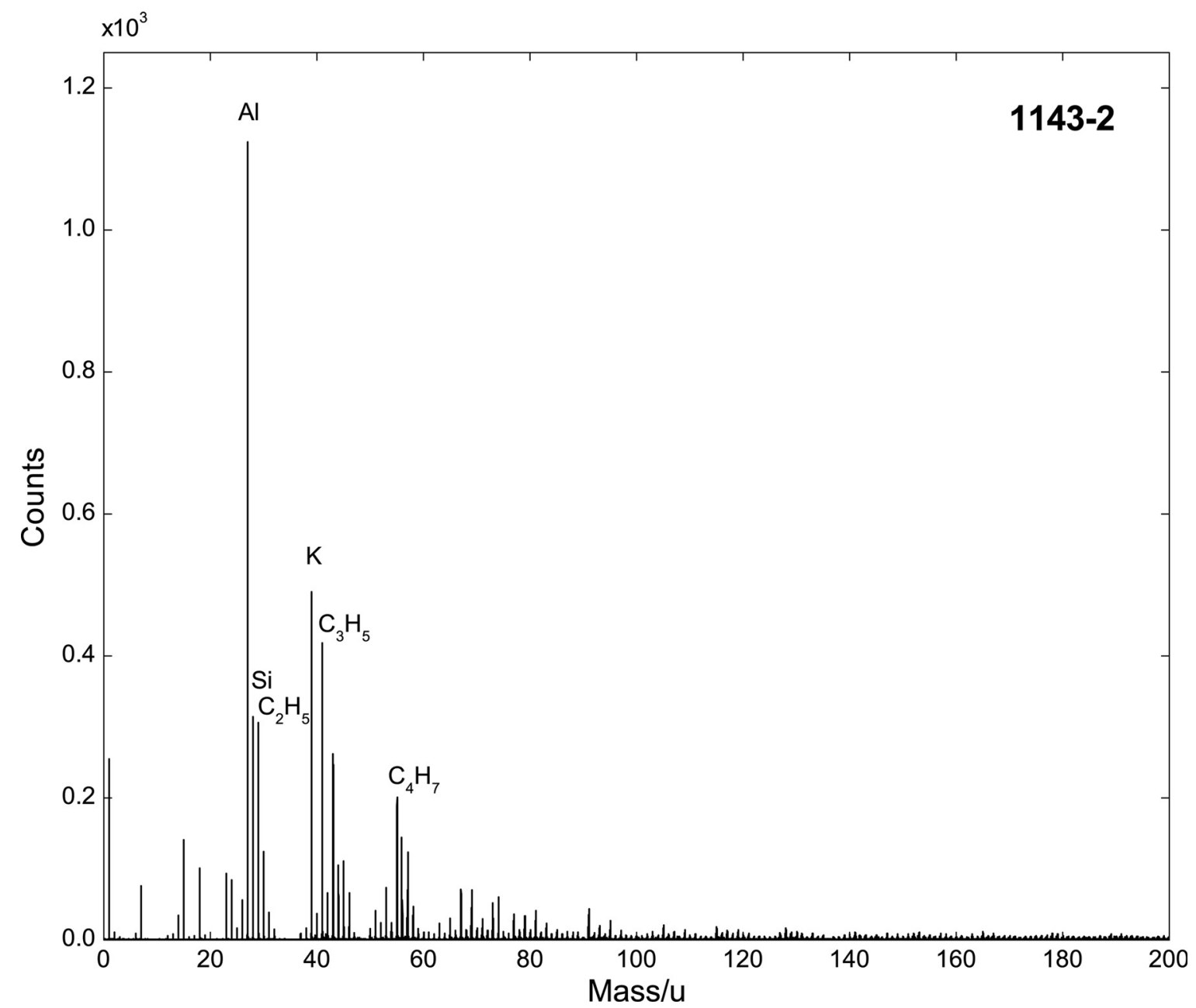

\begin{tabular}{rc}
\hline Accession \# & 01143-02 \\
\hline Host Material & Coal PCT010 \\
Technique & ToF-SIMS \\
Mass Range & 200 Da \\
Instrument & ION-TOF TOF-SIMS IV \\
Analyzer Type & time-of-flight, reflectron \\
Detector Type & $\sim 5000$ \\
Analyzer Mass Resolution & Electron multiplier \\
Specimen Normal to Analyzer & $0^{\circ}$ \\
Primary Beam lon Gun & ION-TOF \\
Primary Species & Ga \\
Primary lon Pulse Length & $25 \times 10^{-9}$ sec \\
Primary lon Pulse Rate & $10 \mathrm{kHz}$ \\
Net Beam Voltage & 25000 \\
Beam Current & $1.5 \mathrm{nA}$ \\
Beam Diameter & $0.1 \mu \mathrm{m}$ \\
Beam Raster Width & $500 \mu \mathrm{m} \times 500 \mu \mathrm{m}$ \\
Beam Incident Angle & $45^{\circ}\left(45^{\circ}\right.$ effective $)$ \\
Source to Analyzer Angle & $45^{\circ}$ \\
Comment & \\
\hline
\end{tabular}




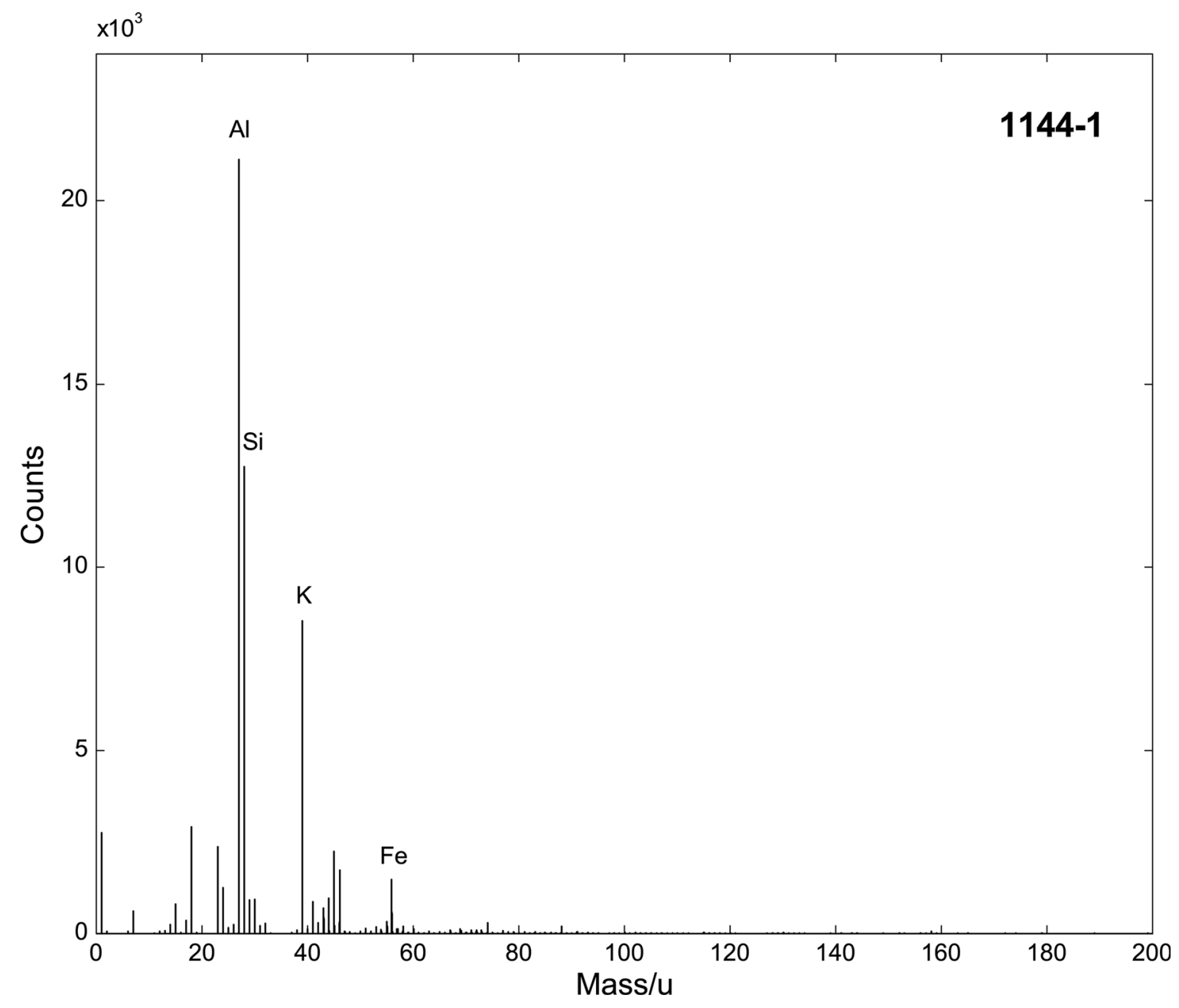

\begin{tabular}{rc}
\hline Accession \# & $\mathbf{0 1 1 4 4 - 0 1}$ \\
\hline Host Material & Coal PCT012 \\
Technique & ToF-SIMS \\
Mass Range & $200 \mathrm{Da}$ \\
Instrument & ION-TOF TOF-SIMS IV \\
Analyzer Type & time-of-flight, reflectron \\
Detector Type & $\sim 5000$ \\
Analyzer Mass Resolution & Electron multiplier \\
Specimen Normal to Analyzer & $0^{\circ}$ \\
Primary Beam lon Gun & ION-TOF \\
Primary Species & Ga \\
Primary lon Pulse Length & $25 \times 10^{-9} \mathrm{sec}$ \\
Primary lon Pulse Rate & $10 \mathrm{kHz}$ \\
Net Beam Voltage & 25000 \\
Beam Current & $1.5 \mathrm{nA}$ \\
Beam Diameter & $0.1 \mu \mathrm{m}$ \\
Beam Raster Width & $500 \mu \mathrm{m} \times 500 \mu \mathrm{m}$ \\
Beam Incident Angle & $45^{\circ}\left(45^{\circ}\right.$ effective $)$ \\
Source to Analyzer Angle & $45^{\circ}$ \\
Comment & \\
\hline
\end{tabular}




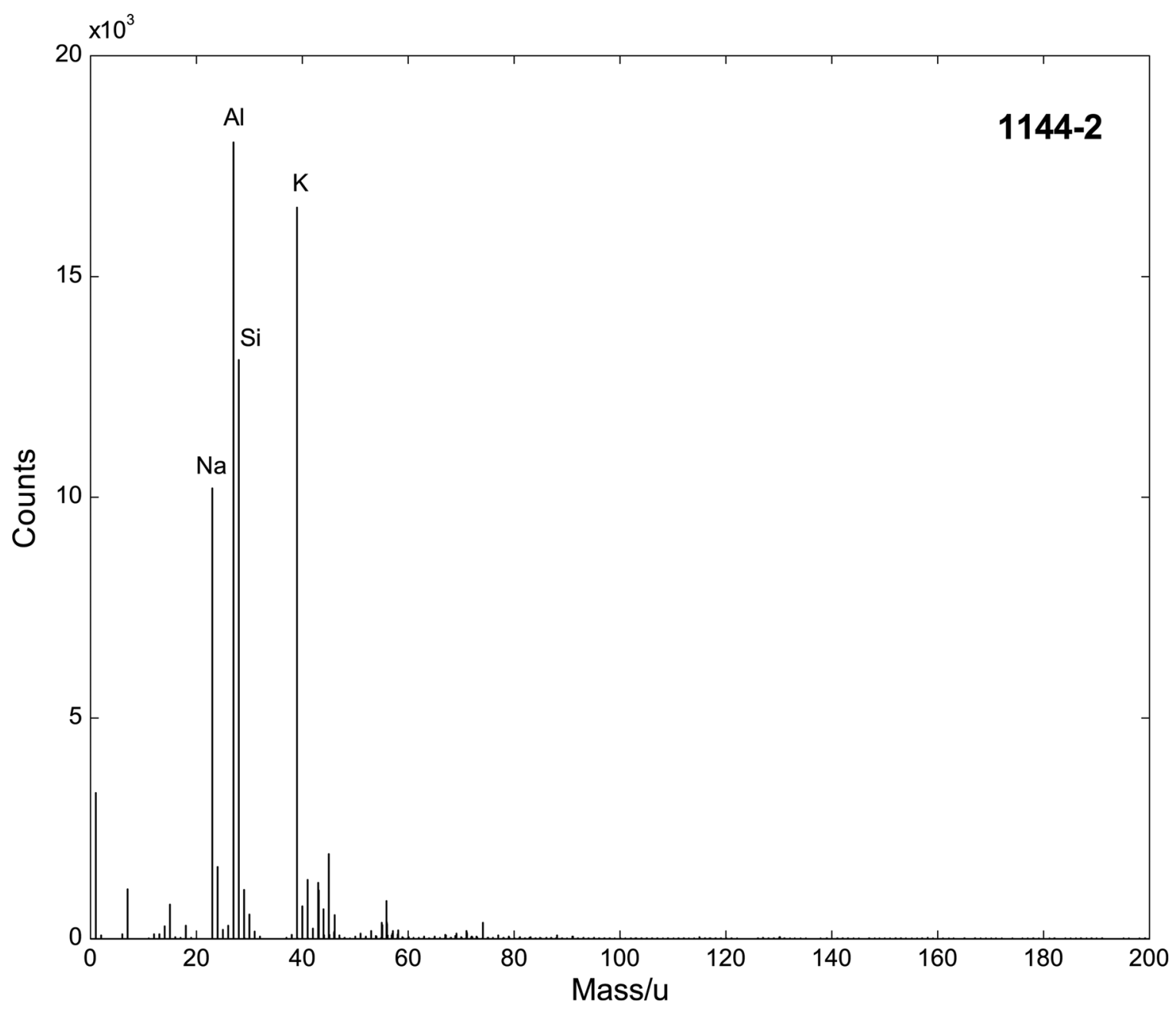

\begin{tabular}{rc}
\hline Accession \# & 01144-02 \\
\hline Host Material & Coal PCT012 \\
Technique & ToF-SIMS \\
Mass Range & $200 \mathrm{Da}$ \\
Instrument & ION-TOF TOF-SIMS IV \\
Analyzer Type & time-of-flight, reflectron \\
Detector Type & $\sim 5000$ \\
Analyzer Mass Resolution & Electron multiplier \\
Specimen Normal to Analyzer & $0^{\circ}$ \\
Primary Beam lon Gun & ION-TOF \\
Primary Species & Ga \\
Primary lon Pulse Length & $25 \times 10^{-9}$ sec \\
Primary lon Pulse Rate & $10 \mathrm{kHz}$ \\
Net Beam Voltage & 25000 \\
Beam Current & $1.5 \mathrm{nA}$ \\
Beam Diameter & $0.1 \mu \mathrm{m}$ \\
Beam Raster Width & $500 \mu \mathrm{m} \times 500 \mu \mathrm{m}$ \\
Beam Incident Angle & $45^{\circ}\left(45^{\circ}\right.$ effective $)$ \\
Source to Analyzer Angle & $45^{\circ}$ \\
Comment & \\
\hline
\end{tabular}




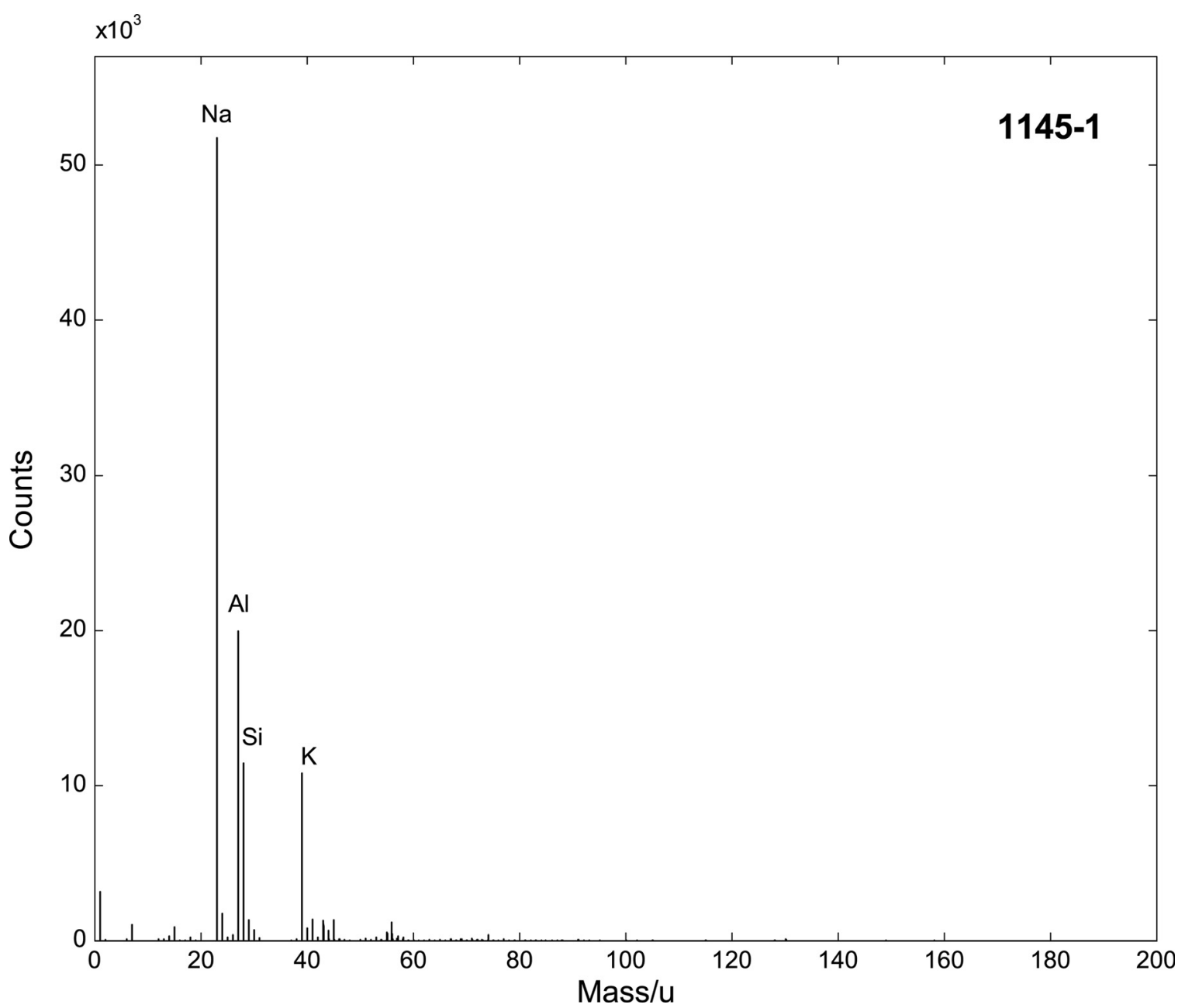

\begin{tabular}{rc}
\hline Accession \# & 01145-01 \\
\hline Host Material & Coal RBL014 \\
Technique & ToF-SIMS \\
Mass Range & $200 \mathrm{Da}$ \\
Instrument & ION-TOF TOF-SIMS IV \\
Analyzer Type & time-of-flight, reflectron \\
Detector Type & $\sim 5000$ \\
Analyzer Mass Resolution & Electron multiplier \\
Specimen Normal to Analyzer & $0^{\circ}$ \\
Primary Beam lon Gun & ION-TOF \\
Primary Species & Ga \\
Primary lon Pulse Length & $25 \times 10^{-9} \mathrm{sec}$ \\
Primary lon Pulse Rate & $10 \mathrm{kHz}$ \\
Net Beam Voltage & 25000 \\
Beam Current & $1.5 \mathrm{nA}$ \\
Beam Diameter & $0.1 \mu \mathrm{m}$ \\
Beam Raster Width & $500 \mu \mathrm{m} \times 500 \mu \mathrm{m}$ \\
Beam Incident Angle & $45^{\circ}\left(45^{\circ}\right.$ effective $)$ \\
Source to Analyzer Angle & $45^{\circ}$ \\
Comment & \\
\hline
\end{tabular}




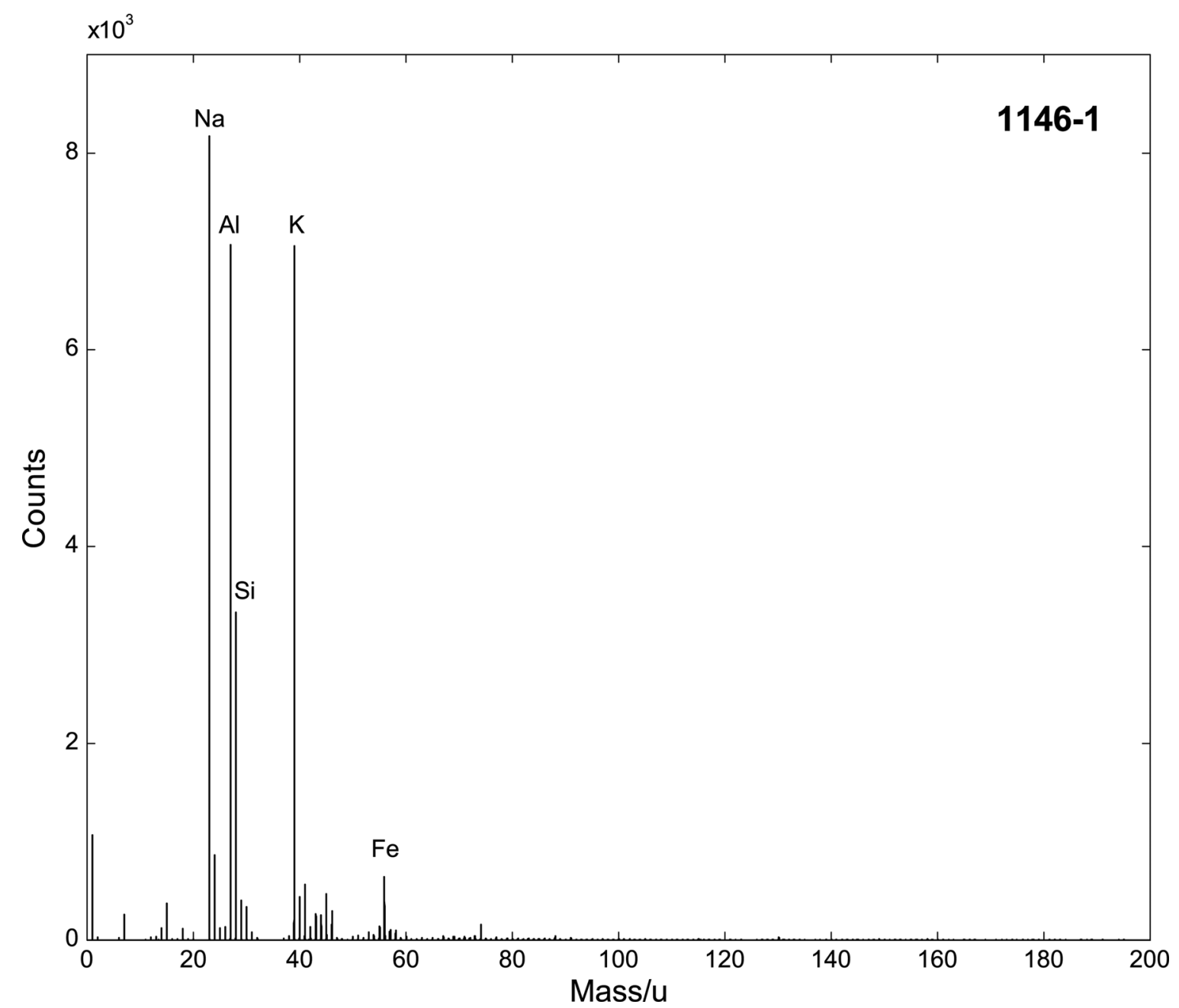

\begin{tabular}{rc}
\hline Accession \# & 01146-01 \\
\hline Host Material & Coal RLL012 \\
Technique & ToF-SIMS \\
Mass Range & 200 Da \\
Instrument & ION-TOF TOF-SIMS IV \\
Analyzer Type & time-of-flight, reflectron \\
Detector Type & $\sim 5000$ \\
Analyzer Mass Resolution & Electron multiplier \\
Specimen Normal to Analyzer & $0^{\circ}$ \\
Primary Beam lon Gun & ION-TOF \\
Primary Species & Ga \\
Primary lon Pulse Length & $25 \times 10^{-9}$ sec \\
Primary lon Pulse Rate & $10 \mathrm{kHz}$ \\
Net Beam Voltage & 25000 \\
Beam Current & $1.5 \mathrm{nA}$ \\
Beam Diameter & $0.1 \mu \mathrm{m}$ \\
Beam Raster Width & $500 \mu \mathrm{m} \times 500 \mu \mathrm{m}$ \\
Beam Incident Angle & $45^{\circ}\left(45^{\circ}\right.$ effective $)$ \\
Source to Analyzer Angle & $45^{\circ}$ \\
Comment & \\
\hline
\end{tabular}




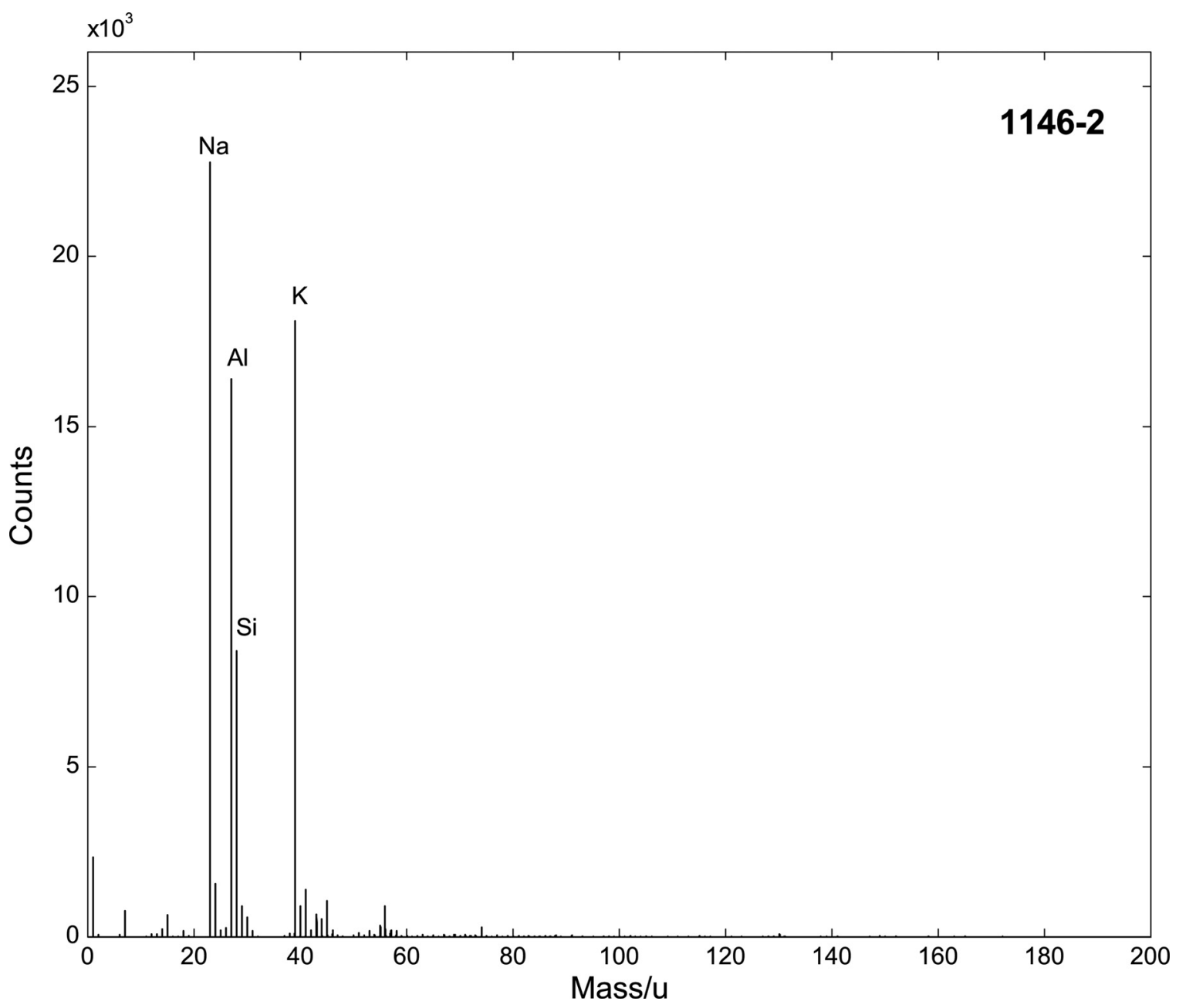

\begin{tabular}{rc}
\hline Accession \# & 01146-02 \\
\hline Host Material & Coal RLL012 \\
Technique & ToF-SIMS \\
Mass Range & $200 \mathrm{Da}$ \\
Instrument & ION-TOF TOF-SIMS IV \\
Analyzer Type & time-of-flight, reflectron \\
Detector Type & $\sim 5000$ \\
Analyzer Mass Resolution & Electron multiplier \\
Specimen Normal to Analyzer & $0^{\circ}$ \\
Primary Beam lon Gun & ION-TOF \\
Primary Species & $\mathrm{Ga}^{+}$ \\
Primary lon Pulse Length & $25 \times 10^{-9} \mathrm{sec}$ \\
Primary lon Pulse Rate & $10 \mathrm{kHz}$ \\
Net Beam Voltage & 25000 \\
Beam Current & $1.5 \mathrm{nA}$ \\
Beam Diameter & $0.1 \mu \mathrm{m}$ \\
Beam Raster Width & $500 \mu \mathrm{m} \times 500 \mu \mathrm{m}$ \\
Beam Incident Angle & $45^{\circ}\left(45^{\circ}\right.$ effective $)$ \\
Source to Analyzer Angle & $45^{\circ}$ \\
Comment & \\
\hline
\end{tabular}




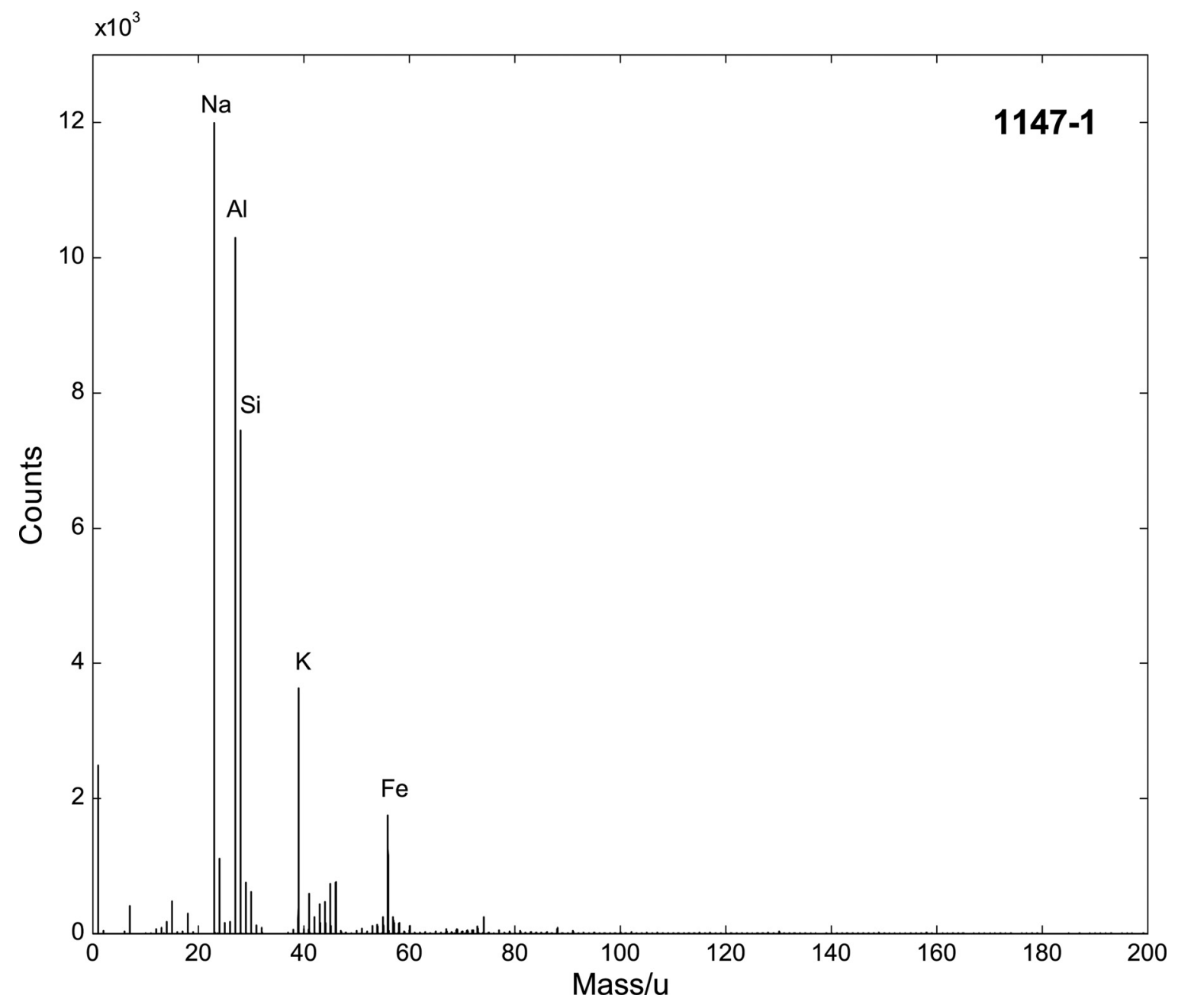

\begin{tabular}{rc}
\hline Accession \# & 01147-01 \\
\hline Host Material & Coal RLL014 \\
Technique & ToF-SIMS \\
Mass Range & 200 Da \\
Instrument & ION-TOF TOF-SIMS IV \\
Analyzer Type & time-of-flight, reflectron \\
Detector Type & $\sim 5000$ \\
Analyzer Mass Resolution & Electron multiplier \\
Specimen Normal to Analyzer & $0^{\circ}$ \\
Primary Beam lon Gun & ION-TOF \\
Primary Species & Ga \\
Primary lon Pulse Length & $25 \times 10^{-9} \mathrm{sec}$ \\
Primary lon Pulse Rate & $10 \mathrm{kHz}$ \\
Net Beam Voltage & 25000 \\
Beam Current & $1.5 \mathrm{nA}$ \\
Beam Diameter & $0.1 \mu \mathrm{m}$ \\
Beam Raster Width & $500 \mu \mathrm{m} \times 500 \mu \mathrm{m}$ \\
Beam Incident Angle & $45^{\circ}\left(45^{\circ}\right.$ effective $)$ \\
Source to Analyzer Angle & $45^{\circ}$ \\
Comment & \\
\hline
\end{tabular}




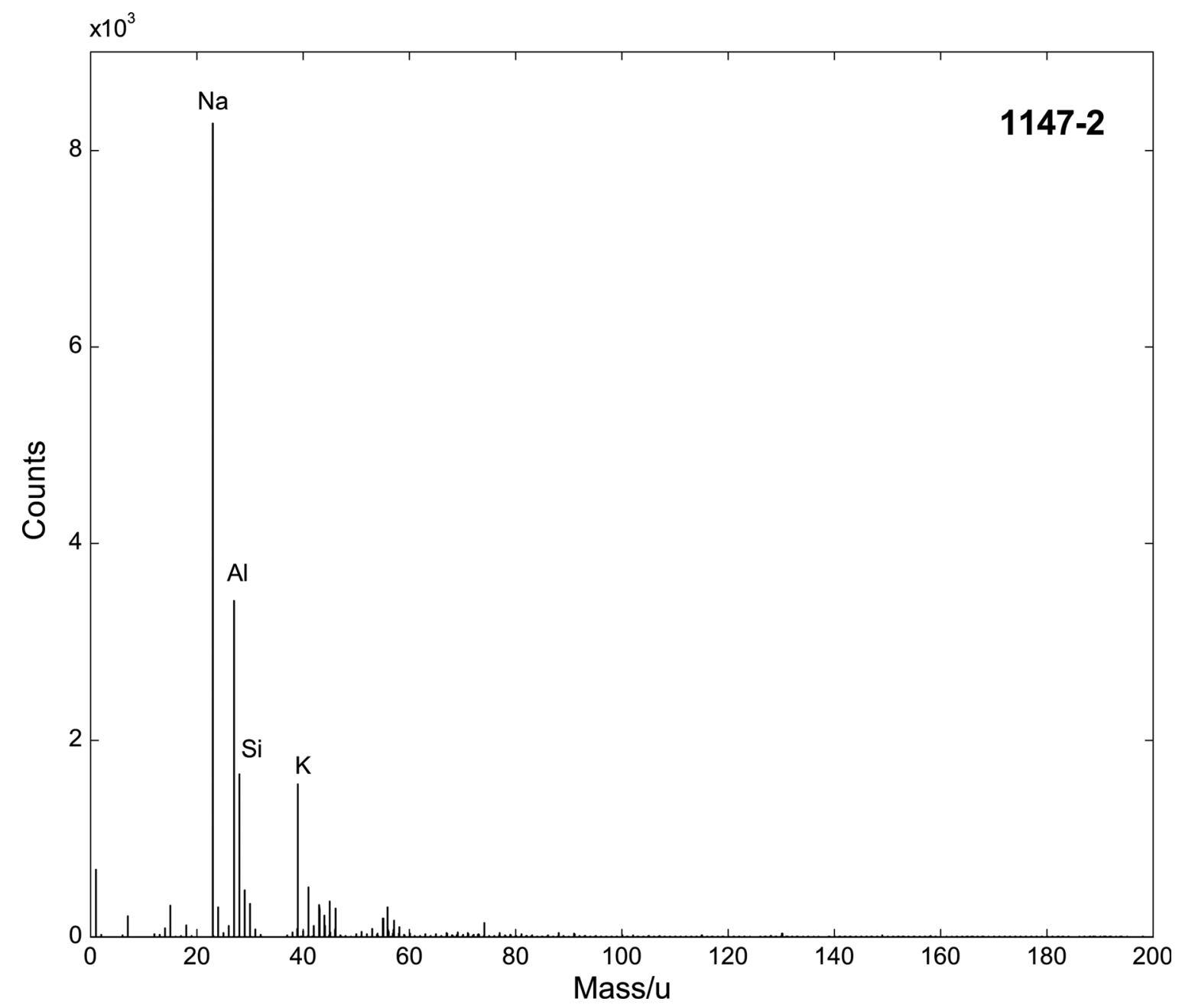

\begin{tabular}{rc}
\hline Accession \# & 01147-02 \\
\hline Host Material & Coal RLL014 \\
Technique & ToF-SIMS \\
Mass Range & $200 \mathrm{Da}$ \\
Instrument & ION-TOF TOF-SIMS IV \\
Analyzer Type & time-of-flight, reflectron \\
Detector Type & $\sim 5000$ \\
Analyzer Mass Resolution & Electron multiplier \\
Specimen Normal to Analyzer & $0^{\circ}$ \\
Primary Beam lon Gun & ION-TOF \\
Primary Species & $\mathrm{Ga}^{+}$ \\
Primary lon Pulse Length & $25 \times 10^{-9} \mathrm{sec}$ \\
Primary lon Pulse Rate & $10 \mathrm{kHz}$ \\
Net Beam Voltage & 25000 \\
Beam Current & $1.5 \mathrm{nA}$ \\
Beam Diameter & $0.1 \mu \mathrm{m}$ \\
Beam Raster Width & $500 \mu \mathrm{m} \times 500 \mu \mathrm{m}$ \\
Beam Incident Angle & $45^{\circ}\left(45^{\circ}\right.$ effective $)$ \\
Source to Analyzer Angle & $45^{\circ}$ \\
Comment & \\
\hline
\end{tabular}

